\title{
ALCOHOL AND THE DYSREGULATION OF COGNITIVE CONTROL: EXPLORING THE ROLE OF EMOTION
}

BY

THOMAS ALLEN SCOTT-SMITH

\author{
A thesis \\ submitted to the Victoria University of Wellington \\ in fulfillment of the requirements for the degree of \\ Master of Science in \\ Cognitive and Behavioural Neuroscience
}

Victoria University of Wellington

2019 



\begin{abstract}
Alcohol consumption attenuates both the behavioural adjustments and the heightened activity in the anterior cingulate cortex (ACC) which are normally observed following errors, leading to the hypothesis that alcohol disrupts the ability to effectively regulate the use of cognitive control. It has furthermore been theorized that these deficits may occur because alcohol reduces the negative affect elicited by unfavourable events, such as errors, thereby weakening the motivation to utilize cognitive control to improve performance. The aim of the current thesis was to provide an empirical test of this model. I carried out two studies in which I examined changes in two physiological indices of affective processing, skin conductance and heart rate, as well as behavioural and EEG responses, following errors on a flanker task. The first study was conducted on sober participants, in order to validate my experimental paradigm, while the second compared the physiological and behavioural effects of errors in participants given either alcohol or a placebo. In both experiments in both experiments, errors produced increased skin conductance responses and heart rate deceleration, and a typical error-related negativity in EEG. However, contrary to what would be expected if alcohol reduced the negative affect generated by errors, no difference in skin conductance or heart rate responses to errors were observed between alcohol and placebo participants in the second study. Furthermore, although intoxicated participants displayed an overall reduction in the use of cognitive control, based on both behavioural (flanker interference) and EEG (occipital alpha power) measures, groups did not differ in the degree to which this control was upregulated immediately after task errors. However, exploratory analyses of EEG indices (the feedback-related negativity and midfrontal theta power) of ACC activity following errors were significantly diminished in intoxicated participants. Overall, these findings suggest that alcohol does not reduce the immediate negative emotional
\end{abstract}


consequences of errors but may instead disrupt brain networks needed for the sustained engagement of cognitive control and attention to task performance. 


\section{Acknowledgements}

Firstly, I must thank my wonderful supervisor Dr. Gina Grimshaw. I am grateful to you not only for providing excellent advice throughout the course of the year, and for broadening my understanding of cognitive psychology, but also for your consistent encouragement and confidence in my abilities, combined with ample tolerance for my lessthan-stellar time management skills. You possess a rare abundance of both intelligence and kindness.

I am also very grateful to Shannon Garland and Dayna Mercer. Thank you for all of your help in running the experiment. My intended sample size was stupidly ambitious, and I never would have come close to reaching it without your assistance. I must furthermore thank Hazel, Sophie, Amy, Daniel, Elliott, Hannah, Kieran, Sumaya and Andrè for the additional support you provided with data collection. I was repeatedly humbled by your collective generosity in regards to your time. Further thanks go to Michael Tooley for freely sharing your considerable technical expertise whenever I had a problem with temperamental recording equipment and unintuitive software. Sorry for all the times I left a mess in the EEG lab or forgot to make a booking.

I would also like to thank my family and my friends. Mum, Dad and Kristin - this hasn't been an easy year for any of us, but I'm grateful to have always been able to count on your love and support. Thank you for listening to all my monologues about the brain, and for never trying to convince me to get a real job. Roland, Chris, Laue and Ben - you guys are awesome and I hope we will be able to spend more time together now.

Finally, I am very grateful for the financial support I received from Victoria University of Wellington, in the form of a Master's (by thesis) scholarship. 


\section{Table of Contents}

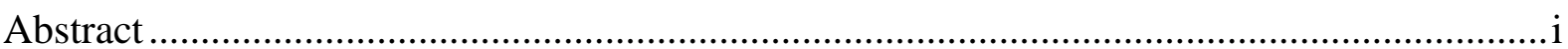

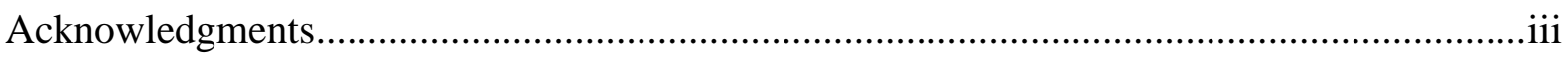

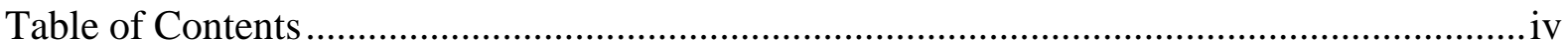

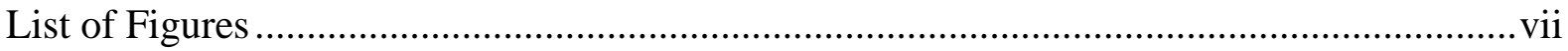

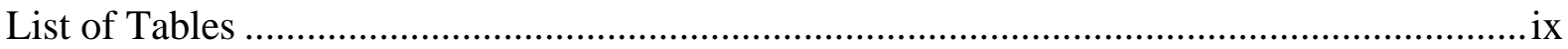

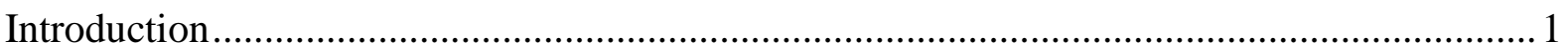

Evaluative Cognitive Control and the Anterior Cingulate Cortex .............................. 2

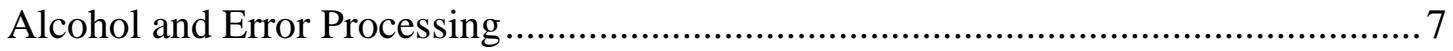

Could Emotion Mediate Alcohol's Effects on Evaluative Control? .......................... 9

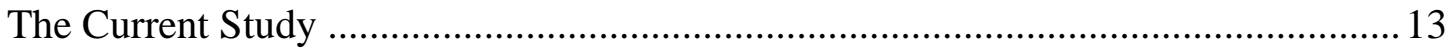

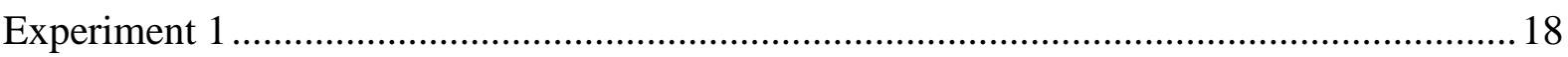

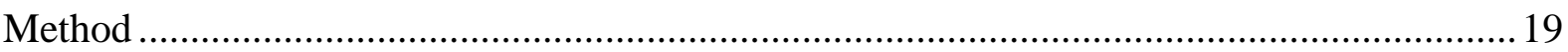

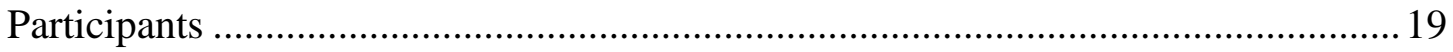

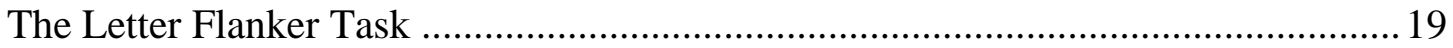

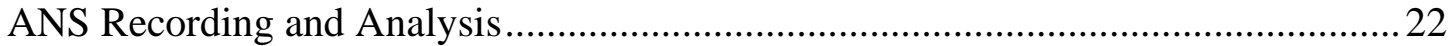

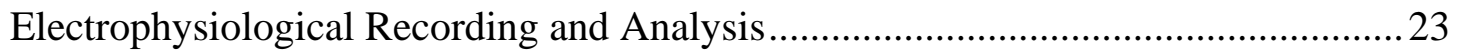

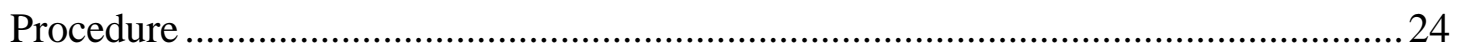

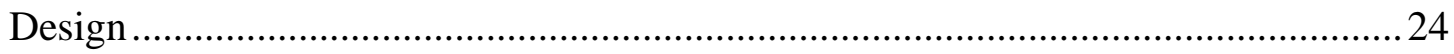

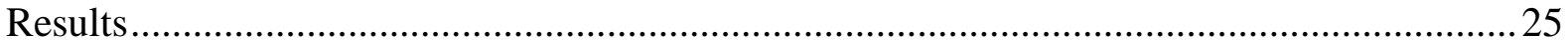

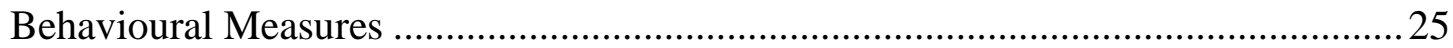

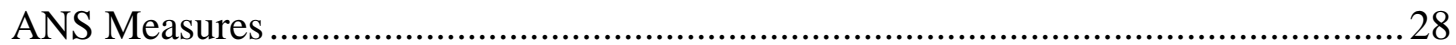

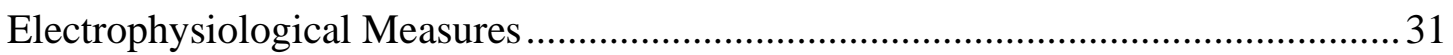

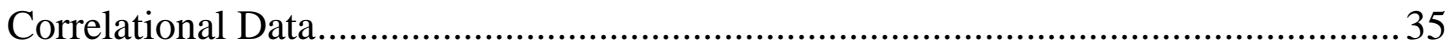

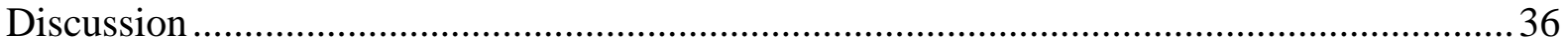




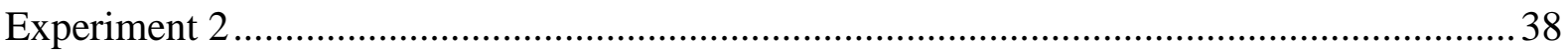

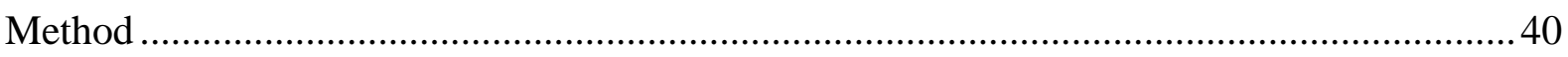

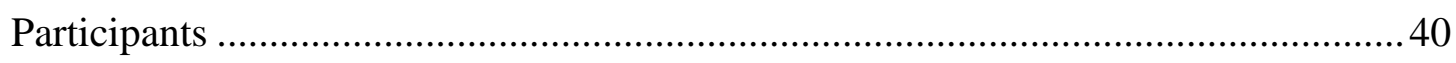

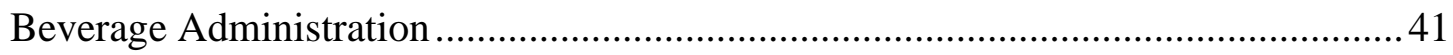

The Positive and Negative Affect Scale …............................................................. 42

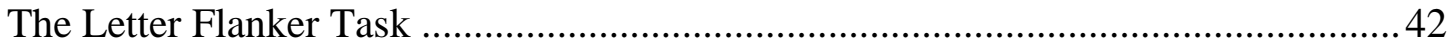

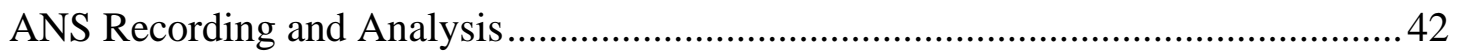

Electrophysiological Recording and Analysis ..................................................... 43

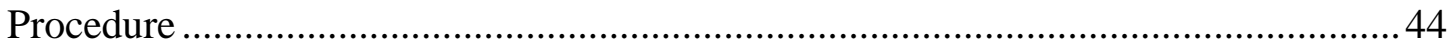

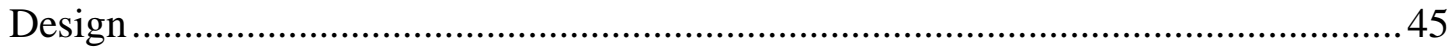

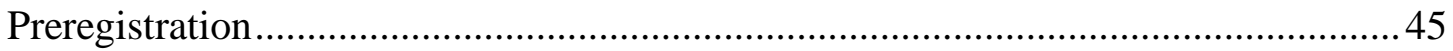

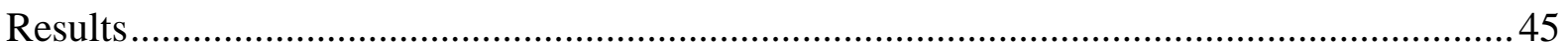

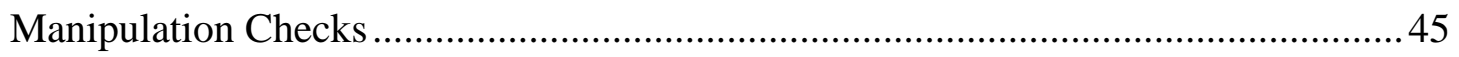

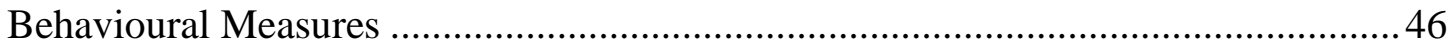

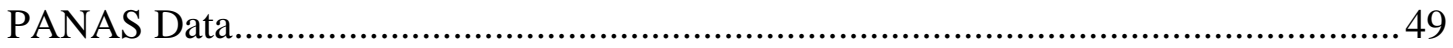

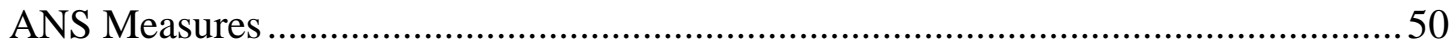

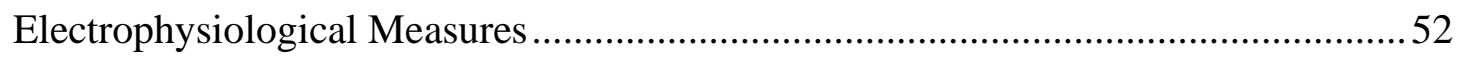

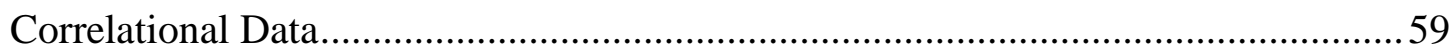

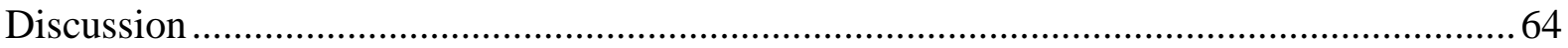

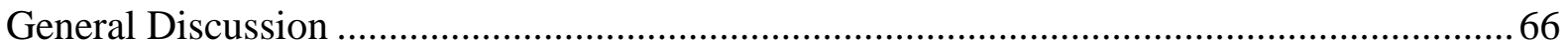

Why No Effect of Alcohol on Post-Error Adaptation? .............................................68

Why No Effect of Alcohol on the ERN? ........................................................... 71

Why No Effect of Alcohol on Negative Affect? ................................................... 72

Why No Correlations Between ANS and Behavioural Responses?........................... 74

Implications for Understanding Alcohol's Effects on Cognition .............................. 75 
Directions for Future Research .......................................................................... 78

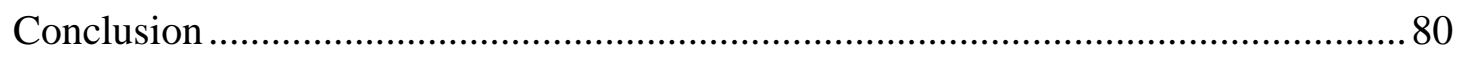

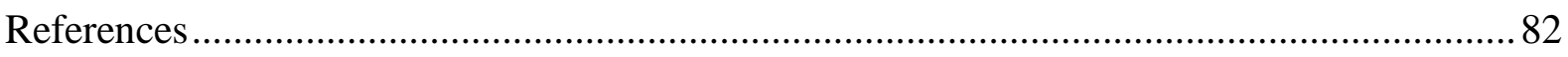

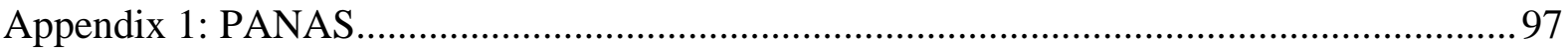

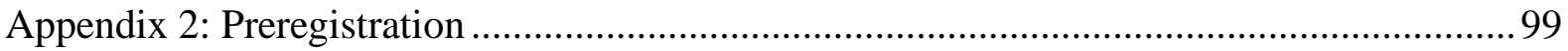




\section{List of Figures}

Figure 1. Schematic of trial procedure in the letter flanker task.

Figure 2. Mean percentage of error responses in the flanker task to congruent and incongruent flanker stimuli (Experiment 1)

Figure 3. Mean RT in the flanker task for correct responses to congruent and incongruent flanker stimuli (Experiment 1).....

Figure 4. Mean RT in the flanker task for correct responses, following a correct response on or an error on the previous trial (Experiment 1)

Figure 5. Size of flanker interference effect for correct responses, following a correct response or an error on the previous trial (Experiment 1)

Figure 6. Mean log-transformed skin conductance responses made following correct responses and errors (Experiment 1) 28

Figure 7. Mean change in heart rate from pre-response baseline, over the $3 \mathrm{~s}$ following correct responses and errors (Experiment 1)

Figure 8. Graphs of SCR and HR responses following correct and erroneous responses made to incongruent stimuli only (Experiment 1$)$

Figure 9. Event related potentials elicited by correct responses and errors, at the FCz electrode (Experiment 1)

Figure 10. Event related potentials elicited by correct responses and errors, at the $\mathrm{Fz}$ and $\mathrm{Cz}$ electrodes (Experiment 1)

Figure 11. Scalp distributions of voltage difference between errors and correct responses, in the $100 \mathrm{~ms}$ period following a response (Experiment 1$)$

Figure 12. Event related potentials elicited by correct responses and errors made to incongruent stimuli only, at the FCz electrode (Experiment 1) 34 
Figure 13. Mean RT in the flanker task, for correct responses to congruent and incongruent stimuli, within each condition (Experiment 2)

Figure 14. Mean RT in the flanker task for correct responses, following a correct response or an error on the previous trial, within each condition (Experiment 2) 48

Figure 15. Size of flanker interference effect for correct responses, following a correct response or an error on the previous trial, within each condition (Experiment 2). 49

Figure 16. Mean log-transformed skin conductance responses made to correct responses and errors, within each condition (Experiment 2) 51

Figure 17. Mean change in HR (BPM) from pre-response baseline over the $3 \mathrm{~s}$ following correct responses and errors, within each condition (Experiment 2)

Figure 18. Event related potentials elicited by correct responses and errors, within each condition, at the FCz electrode (Experiment 2)

Figure 19. Heatmaps displaying power estimates produced by time-frequency analysis of correct responses and errors, within each condition, at the FCz electrode (Experiment 2)

Figure 20. Plot tracking theta power over the $2000 \mathrm{~ms}$ before and after a correct response or an error, within each condition, at the FCz electrode (Experiment 2)..... 56

Figure 21. Heatmaps displaying power estimates produced by time-frequency analysis of correct responses and errors, within each condition, at the Oz electrode (Experiment 2) 58

Figure 22. Plot tracking alpha power over the $2000 \mathrm{~ms}$ before and after a correct response or an error, within each condition, at the Oz electrode (Experiment 2) 59 


\section{List of Tables}

Table 1. Correlations between psychophysiological measures, and post-error reduction of interference and post-error slowing (Experiment 1)

Table 2. Mean percentage of each response type within each condition (Experiment 2) 47

Table 3. Mean ratings for positive and negative affect items on the PANAS, upon entering the lab and after beverage administration, for participants in each condition (Experiment 2)

Table 4. Correlations between psychophysiological responses to errors, changes in PANAS ratings, and measures of post-error adaptation (Experiment 2)

Table 5. Correlations between psychophysiological responses to errors, changes in PANAS ratings, and measures of post-error adaptation for participants within the placebo group only (Experiment 2)

Table 6. Correlations between psychophysiological responses to errors, changes in PANAS ratings, and measures of post-error adaptation for participants within the alcohol group only (Experiment 2) 


\section{Alcohol and the Dysregulation of Cognitive Control: Exploring the Role of Emotion}

The consumption of alcohol can often lead to dramatic changes in behaviour, sometimes with dire consequences, including interpersonal aggression, unsafe sexual practices and motor vehicle accidents (Ker \& Ivers, 2006; Kypri, Paschall, Langley, Baxter, Cashell-Smith \& Bourdeau, 2009). Alcohol is a central nervous system depressant which acts primarily through agonism of the inhibitory $\mathrm{GABA}_{\mathrm{A}}$ receptor (Lobo \& Harris, 2008). It is therefore somewhat paradoxical that is should promote reckless and imprudent behaviour along with sedation and ataxia. It has been suggested that the behavioural dysregulation produced by alcohol may stem from a reduced capacity to exercise cognitive control (Casbon, Curtin, Lang, \& Patrick, 2003; Curtin \& Fairchild, 2003), the collection of top-down processes which allow us to adjust our actions based on our current goals, rather than rely solely on automatic or habitual responses (Alexander \& Brown, 2010; Botvinick, Braver, Barch, Carter, \& Cohen, 2001).

One line of evidence which seemingly supports this view is the pronounced effect of alcohol on error processing. Making an error demonstrates that current control settings are suboptimal, and typically prompts increased activity in brain regions implicated in the management of cognitive control, as well as behavioural changes indicating increased use of control (Carter, Braver, Barch, Botvinick, Noll \& Cohen 1998; Danielmeier \& Ullsperger, 2011). Numerous studies have found that alcohol significantly diminishes both neural and behavioural markers of post-error control adjustment (Bailey, Bartholow, Saults \& Lust; 2014; Marinkovic, Rickenbacher, Azma, Artsy \& Lee, 2013; Ridderinkhof, de Vlugt, Bramlage, Spaan, Elton, Snel \& Band, 2002), leading to the conclusion that alcohol impairs the ability to monitor and dynamically adjust the use of cognitive control based on the needs of the current situation. 
While the effects of alcohol on post-error adaptation provide some insight into how alcohol might lead to changes in behaviour, the actual mechanism behind these phenomena, and their relationship to the real-world effects of alcohol, remains unclear. Considering that recent theoretical perspectives have highlighted the importance of emotion in facilitating cognitive control (Inzlicht, Bartholow \& Hirsch, 2015; Saunders, Lin, Milyavskaya \& Inzlicht, 2017), a possible answer to these questions may lie in the well-known anxiolytic and stress-dampening effects of alcohol (e.g. Donohue, Curtin, Patrick, \& Lang, 2007. Bartholow and colleagues (2012) propose that alcohol disrupts post-error adjustments in cognitive control because it reduces negative affect, arguing that intoxication makes failure less distressing, thereby weakening the drive to utilize cognitive control to improve performance following an error.

In this thesis I present two experiments designed to test whether a reduction in negative affect by alcohol could plausibly account for failures to upregulate cognitive control after an error. The first experiment examined affective responses to errors, via psychophysiology, in sober participants, to validate my experimental paradigm, and explore the general relationship between emotional response and post-error adaptation. The second experiment repeated this procedure on participants given either alcohol or placebo, to determine whether alcohol reduces the negative affect elicited by errors, and if this mediates the observed effects of alcohol on post-error adaptation.

\section{Evaluative Cognitive Control and the Anterior Cingulate Cortex}

Research into top-down control of behaviour has traditionally focused on understanding how specific control processes, such as inhibition, maintenance and set-shifting, work to modulate cognition and action (e.g. Miyake, Friedman, Emerson, Witzki, Howerter \& Wager, 2000). However, a complete theory of cognitive control must also account for the mechanisms by which the need for control is detected and adjustments are made as necessary, 
a process sometimes referred to as evaluative cognitive control (Botvinick et al., 2001). Although the computational basis for evaluative control remains hotly contested (Alexander \& Brown, 2010), there is a general consensus among contemporary theorists that, neurobiologically, the regulation of cognitive control is facilitated primarily though the anterior cingulate cortex (ACC; Botvinick et al., 2001; Cavanagh \& Frank, 2014; Holroyd \& Yeung, 2012; Shenhav, Cohen \& Botvinick, 2016). The ACC is heavily interconnected with lateral prefrontal regions involved in implementing specific control strategies (Kouneiher, Charron \& Koechlin, 2009; Miller \& Cohen, 2001). It is believed that in situations where increased cognitive control would be beneficial, the ACC transmits a signal to these prefrontal areas indicating the type and level of control required (Shenhav et al., 2016). It has furthermore been postulated that electrical oscillations between 4 and $8 \mathrm{~Hz}$, manifesting as midfrontal theta waves in electroencephalography (EEG) recordings, form the biophysical basis for this ACC control signal (Cavanagh \& Frank 2014: Cavanagh, Zambrano-Vazquez \& Allen, 2012).

One scenario which demands the increased engagement of cognitive control is the occurrence of conflict between mutually incompatible mental representations or behavioural responses (see Botvinick et al., 2001). For example, in an Eriksen flanker task (Eriksen \& Eriksen, 1974) participants must respond to a central target stimulus, usually an arrow or a letter, which is flanked by additional, non-target stimuli. These flankers will sometimes prompt a different response than the target, leading to interference in the form of increased response times or decreased accuracy. To minimize this interference participants must utilize cognitive control to inhibit the incorrect responses cued by incongruent flankers. Other experimental tasks used to study conflict and control include the Stroop task, in which the colour of word stimuli conflict with their meaning, and the go/no-go task, in which an overlearned response conflicts with a cue to withhold that response. 
EEG studies of conflict tasks typically observe an event-related potential, the anterior $\mathrm{N} 2$, around 200ms after the presentation of a conflict provoking stimulus, such as an incongruent flanker array (e.g. Donkers \& Van Boxtel, 2004; Nieuwenhuis, Yeung, Van Den Wildenberg, \& Ridderinkhof, 2003; Veen \& Carter, 2002). Consistent with its putative role in upregulating control, the ACC has been identified as the likely source of the N2 (Bekker, Kenemans \& Verbaten, 2005; Jonkman, Sniedt \& Kemner, 2007). Increased ACC activation by conflict has also been observed using fMRI (Carter, Braver, Barch, Botvinick, Noll \& Cohen, 1998; Nee, Wager, \& Jonides, 2007). Furthermore, a study by Kerns and colleagues (2004) found that, within a Stroop task, greater ACC activation to incongruent stimuli predicted reduced Stroop interference, providing evidence that the ACC plays a causal role in facilitating cognitive control. The same study also examined conflict adaptation, the tendency for the control of interference to improve with the repeated presentation of conflict stimuli. They found that while ACC activity initially increased when incongruent stimuli were presented, activity decreased when conflict adaptation took place. This supports the idea that the function of the ACC is to signal for increased control, rather than carry out specific control strategies. The importance of the ACC is in regulating cognitive control is further evinced by lesion studies showing that damage to the ACC significantly weakens conflict adaptation (Newman, Creer \& McGaughy, 2015; Sheth et al., 2012).

In addition to conflict, the ACC is also sensitive to errors. Numerous EEG studies have detected a large negative deflection in voltage across midfrontal electrodes occurring approximately $80 \mathrm{~ms}$ after error commission in speeded-response tasks (e.g. Boksem, Tops, Wester, Meijman, \& Lorist, 2006; Gehring, Goss, Coles, Meyer \& Donchin, 1993; Scheffers, Coles, Bernstein, Gehring, \& Donchin, 1996). This ERP has been dubbed the error-related negativity (ERN), and, like the anterior N2, is thought to be generated within the ACC (Herrmann, Römmler, Ehlis, Heidrich \& Fallgatter, 2004; Miltner, Lemke, Weiss, Holroyd, 
Scheffers, \& Coles, 2003). The short latency of the ERN suggests that it reflects the operation of an endogenous monitoring system which evaluates the likely outcome of motor commands, rather a response to external performance cues (Allain, Hasbroucq, Burle, Grapperon \& Vidal, 2004). However, a related, but distinct, ERP component known as the feedback-related negativity (FRN) is also observed when subjects are given explicit feedback after they make an error, typically within 200 to 400ms (e.g. Miltner, Braun \& Coles, 1997; Olvet \& Hajcak, 2009). Conflict and error responses within the ACC are thought to reflect the operation of a single underlying evaluative control system (Botvinick et al., 2001; Shenhav et al., 2016); just as additional control is needed to resolve response conflict, making an error is a sign that the current level of control is inadequate for successful task performance, and should therefore be increased. Testing subjects on a battery of different tasks, Cavanagh, Zambrano-Vazquez and Allen (2012) found that variance in the N2, ERN and FRN components could parsimoniously accounted for by a single underlying band of midfrontal theta oscillations. This anatomical convergence between conflict and error-related ERPs provides compelling evidence for a domain-general signal for the increase of cognitive control, generated within the ACC.

The idea that error-related ERPs like the ERN reflect a call for greater control from within the ACC is further supported by behavioural and EEG evidence that cognitive control is upregulated immediately after an error. Firstly, when participants make errors on speeded-response tasks, such as the flanker task and the Stroop task, their overall response time typically increases on the next trial (e.g. Debener, Ullsperger, Siegel, Fiehler, Von Cramon \& Engel, 2005; King, Korb, von Cramon \& Ullsperger, 2010; Laming, 1979) This phenomenon is referred to as post-error slowing (PES) and is thought to reflect a deliberate attempt to increase accuracy at the expense of speed (Danielmeier \& Ullsperger, 2011; but see Notebaert, Houtman, Van Opstal, Gevers, Fias \& Verguts, 2009). While this suggests a 
shift in control strategy, rather than an increase in control per se, it nevertheless implicates the evaluative control system. A more direct source of behavioural evidence for increased cognitive control following errors is post-error reduction of interference (PERI), a tendency for the interference with response time elicited by conflict stimuli, such as incongruent Stroop words, to decline on trials immediately following an error (e.g. De Bruijn, Hulstijn, Verkes, Ruigt \& Sabbe, 2004; King et al., 2010; Ridderinkhof, 2002). As discussed previously, effective use of cognitive control minimizes the degree to which conflict stimuli compete with task-relevant information, thereby producing interference. The existence of PERI thus indicates that errors prompt a rapid upregulation of control. Additionally, a meta-analysis by Cavanagh and Shackman (2016) reached the conclusion that the size of behavioural adjustments following errors is consistently predicted by the magnitude of the ERN, further linking the ACC to the dynamic adjustment of cognitive control.

Behavioural evidence of for the post-error increase of cognitive control is reinforced by a number of EEG studies demonstrating the suppression of alpha wave activity following errors (Carp \& Compton, 2009; Compton, Bissey \& Worby-Selim, 2014; van Driel, Ridderinkhof \& Cohen, 2012). Alpha waves are electrical oscillations between 8 and 13Hz, which vary inversely with overall cortical activity (Basar, 2012). Alpha waves are thought to act as a mechanism for selective attention, increasing in order to minimize processing of irrelevant information, while in decreasing in appropriate cortical regions when there is a need to engage with goal-relevant stimuli. (Foxe, Simpson \& Ahlfors, 1998). For instance, when subjects perform visual tasks, alpha power in occipital areas will decline when they focus on a specific target. (Fu, Foxe, Murray, Higgins, Javitt \& Schroeder, 2001; Klimesch, Doppelmayr, Russegger, Pachinger \& Schwaiger, 1998). The phasic suppression of alpha waves following an error is therefore consistent with the increased utilization of cognitive control, in order to support attention to task-relevant stimuli. Taking all existing research into 
account, a strong case can be made that errors prompt an increase of cognitive control, and that this is primarily facilitated through the ACC.

\section{Alcohol and Error Processing}

Numerous studies have produced evidence that alcohol can impair cognitive control processes (e.g. Bartholow, Pearson, Sher, Wieman, Fabiani \& Gratton, 2003; Curtin \& Fairchild, 2003; Fillmore, Vogel-Sprott \& Gavrilescu, 1999), particularly the inhibition of prepotent responses (e.g. Casbon et al., 2003, Nikolaou, Critchley \& Duka, 2013; Rose \&

Duka, 2008). However, alcohol is far from consistent in its effects on control functions. Despite the highly visible effects of alcohol on social behaviour and complex task performance in the real world, multiple studies have found that alcohol, at least at moderate doses, has only a limited impact on many types of cognitive and executive processes (e.g. Boissoneault, Sklar, Prather \& Nixon, 2014; Gustafson \& Kallmen, 1990a, 1990b; Schweizer, Vogel-Sprott, Danckert, Roy, Skakum \& Broderick, 2006; Tarter, Jones, Simpson \& Vega, 1971). This discrepancy may exist because alcohol specifically disrupts evaluative cognitive control, that is, the ability to determine the optimal level of control and adjust as needed. Thus, intoxicated individuals may be able to gradually engage the control settings needed to perform relatively uniform experimental tasks, while still struggling in situations where control demands are constantly changing, such as while driving. This view is supported by a number of studies examining the effects of alcohol on cognitive and neural responses to error commission.

The effect of alcohol on error processing was first investigated by Ridderinkhof and colleagues (2002). In their study, subjects were given either a low dose of alcohol (0.04\% blood alcohol concentration /BAC), a high dose of alcohol (0.1\% BAC) or placebo, and then completed an arrow flanker task, in which incongruent displays were presented on $50 \%$ of trials. They found that subjects receiving either high or low doses of alcohol produced a 
smaller error-related negativity (ERN) after making an error than participants in the placebo group. Furthermore, while placebo group participants displayed PERI, this post-error behavioural adaptation was abolished in intoxicated subjects. Ridderinkhof and colleagues (2002) concluded that alcohol impairs the ability of the ACC to detect action slips such as errors, and thus preventing it from setting control to an appropriate level.

A similar pattern of results was reported by Bailey and colleagues (2014). They administered either an alcoholic $(0.08 \% \mathrm{BAC})$, placebo or control beverage to their participants, then had them complete an arrow flanker task. They not only found that alcohol reduced the amplitude of the ERN, but that on trials following errors, alcohol group participants displayed increased flanker interference and reduced EEG indices of cognitive control, in the form of the anterior $\mathrm{N} 2$ and the negative slow wave, leading them to conclude that alcohol prevented the recovery of cognitive control after a control failure had led to an error.

Marinkovic and colleagues (2013) provide further evidence that alcohol interferes with the ability to utilize control resources to salvage performance after an error. They had subjects perform an anti-saccade task, in which they were required, when cued, to make eye movements in the opposite direction of a target, forcing them to inhibit a natural tendency to orient towards novel stimuli. At the beginning of the experiment participants were given either an alcoholic $(0.06 \%$ BAC for men, $0.055 \%$ BAC for women) or a placebo beverage, and their brain activity was monitored using fMRI while they performed the task. Placebo participants showed increased ACC activation after failing to make an eye movement in the required direction, with this response being attenuated in the alcohol condition. Furthermore, upon making an incorrect eye movement, participants typically attempted to correct their error. However, intoxicated participants were significantly less likely to do this, suggesting 
an impairment in the ability to upregulate control over automatic behaviour when current performance proves inadequate.

While the finding that alcohol prevents post-error behavioural adaptation has not always been successfully replicated (i.e. Bombeke, Schouppe, Duthoo \& Notebaert, 2013), empirical support for the claim that alcohol diminishes ACC responses to errors is robust, having additionally been observed in both EEG (Easdon, Izenberg, Armilio, Yu \& Alain, 2005) and fMRI (Anderson, Stevens, Meda, Jordan, Calhoun, \& Pearlson, 2011) studies examining go/no-go task performance, and in an fMRI study employing a Stroop task (Marinkovic, Rickenbacher, Azma, \& Artsy, 2012). Given the established importance of the $\mathrm{ACC}$ in regulating cognitive control, these findings indicate that alcohol does likely interfere with evaluative control processes in some manner. However, it is currently unclear why alcohol should affect this specific brain system, and to what extent the underlying mechanism is relevant in understanding alcohol-induced behavioural dysregulation in the real world. Ridderinkhof and colleagues (2002) argue that alcohol attenuates the ACC activity elicited by errors because it reduces the likelihood that errors will be noticed. However, Bailey and colleagues (2014; see also Bartholow et al., 2012) found that when asked to report on their performance, intoxicated participants were just as successful as sober participants at identifying when they had committed an error, suggesting that a different mechanism may be driving this well-established effect.

\section{Could Emotion Mediate Alcohol's Effects on Evaluative Control?}

Bartholow and colleagues (2012) offer an alternative explanation for why alcohol might reduce ACC activity and behavioural adaptation following errors. They propose that alcohol reduces the negative affect elicited by errors, and that this in turn reduces the motivation to upregulate cognitive control so that further errors can be avoided. While emotion is often cast as the enemy of self-control, there is now substantial evidence pointing to an important role 
for negative affect in facilitating cognitive control (e.g. Fröber, Stürmer, Frömer \& Dreisbach, 2017; Olvet \& Hajcak, 2008; Steenbergen, Band \& Hommel, 2009). The ERN in particular is known to be highly sensitive to emotional and motivational factors, showing an enhanced amplitude in individuals with high levels of worry, anxiety (Hajcak, McDonald \& Simons, 2003a) and punishment sensitivity (Boksem et al., 2006). Situational factors, such as the short term induction of negative affect (Wiswede, Münte, Goschke \& Rüsseler, 2009), social evaluation (Cavanagh \& Frank, 2008), or a monetary penalty for making errors (Pailing \& Segalowitz, 2004), can also temporarily increase the size of the ERN. Some contemporary theorists go so far as to argue that evaluative cognitive control is an intrinsically emotional process, with control adjustments being enacted in order to alleviate the aversive experience of conflict or failure (Inzlicht et al., 2015, Saunders et al., 2017). Assuming this is true, a decline in negative emotionality while intoxicated could plausibly have a significant impact on the upregulation of cognitive control following errors.

To test their hypothesis, Bartholow and colleagues (2012) carried out a study in which participants, divided into alcohol (0.1\% BAC), placebo and control groups, completed a weapon identification task, in which faces of either black or white men were briefly presented, followed by a picture of a gun or a tool. Participants were then required to identify whether a weapon was presented or not. In this task, participants are typically biased towards making weapon responses after seeing a black face and must therefore employ cognitive control to overcome this automatic racial bias and perform accurately. Bartholow and colleagues found that participants given alcohol were more likely make erroneous weapon responses when primed with a black face. Furthermore, they replicated previous findings by showing that alcohol reduced both the amplitude of the ERN, and the degree to which behavioural measures of control, in this case accuracy, were improved following errors. 
In order to explore the potential role of affect in driving this disruption of evaluative control by alcohol, Bartholow and colleagues (2012) additionally administered a self-report inventory of state affect, the positive and negative affect scale (PANAS), to participants before and after they were given their alcoholic, placebo or control beverage. They found that alcohol, but not placebo or control, reduced participants' level of state negative affect. Using structural equation modelling, they then demonstrated that this reduction in negative affect statistically mediated the decreased behavioural adaptation observed in the alcohol condition. On the basis of these findings, Bartholow and colleagues argue that the dysregulation of cognitive control by alcohol is intrinsically linked to its capacity to reduce the negative emotions generated by aversive events such as errors.

Although the evidence Bartholow and colleagues (2012) provide for their hypothesis is arguably limited and indirect, relying solely on a self-report measure of state negative affect, their model is nonetheless plausible given what we already know about alcohol, emotion and cognitive control. In addition to the growing evidence for a general link between cognitive control and negative affect, the idea that making a mistake is aversive is intuitively sensible and well supported by existing research. Hajcak, McDonald and Simons (2003b) investigated the effect of error commission in a Stroop task on two outputs of the autonomic nervous system (ANS), skin conductance response (SCR) and heart rate (HR). SCR, recorded via monitoring of electrodermal activity, reflects activity in the sympathetic branch of the ANS, and generally increases in response to emotionally arousing stimuli (Codispoti, Bradley, \& Lang, 2001). HR on the other hand, is sensitive to both sympathetic and parasympathetic activity; emotional stimuli tend to elicit an initial parasympathetic-driven deceleration, which reflects attentional orienting, followed by sympathetic-driven acceleration (Codispoti et al., 2001; Bradley, 2009). Hajcak and colleagues found that errors produced an elevated SCR, and a fast deceleration of HR, which was then followed by acceleration. This pattern of ANS 
activity is very similar to what is typically observed in response to aversive stimuli, such as unpleasant pictures (e.g. Bradley, Codispoti, Cuthbert \& Lang 2001; Tooley, Carmel, Chapman \& Grimshaw, 2017). A follow up study (Hajcak, McDonald \& Simons, 2004), not only replicated their original findings, but also showed that SCR and HR deceleration following errors was exaggerated in individuals with high levels of trait negative affect, supporting an interpretation in which ANS changes elicited by errors reflect an emotional response. Furthermore, the enhancement of SCR and HR deceleration by errors has been replicated in several other studies (Fiehler, Ullsperger, Grigutsch \& von Cramon, 2004; O’Connell, Bellgrove, Dockree \& Robertson, 2004; Wessel, Danielmeier \& Ullsperger, 2011).

However, ANS activity represents only one facet of affective processing (Mauss, Levenson, McCarter, Wilhelm \& Gross, 2005) and is known to be more sensitive to the arousal than the valence of an emotional event or stimulus (Bradley, 2009). Nevertheless, there is converging evidence from other experimental paradigms that errors do generate negative affect. Hajcak and Foti (2008) found that the size of the acoustic startle response, a well-established index of negatively-valenced emotion, was increased after subjects made errors in a flanker task. Furthermore, Elkins-Brown, Saunders and Inzlicht (2017) demonstrated that error commission increased electromyographic activity over the corrugator supercilia muscle, likewise consistent with the experience of negative affect.

Finally, Aarts, De Houwer \& Pourtois (2012) found that when participants failed to withhold a response on a go/no-go task, they were subsequently faster at categorizing negativelyvalenced words, compared to when they responded correctly. Taken together, these studies provide strong evidence that errors are indeed aversive.

There is also considerable evidence that alcohol can alleviate negative emotion and weaken affective responses to noxious stimuli. Alcohol has been found to reduce both 
physiological and self-report measures of negative affect across a number of experimental paradigms designed to elicit distress, including the delivery an embarrassing self-disclosing speech (Levenson, Sher, J., Grossman, Newman \& Newlin, 1980; Sher \& Walitzer, 1986), the viewing of unpleasant pictures (Donohue, Curtin, Patrick \& Lang, 2007) and the threat of electric shock (Levenson et al., 1980; Moberg \& Curtin, 2009). However, it is important to note that the capacity of alcohol to blunt negative emotion is not uniform and can vary substantially based on the situation. In particular, the anxiolytic and stress reducing properties of alcohol appear to be modulated by attention: when attention can be diverted from distressing stimuli, negative affect is attenuated, but if attention is instead directed to those stimuli, negative affect is not reduced, and may even increase (Curtin, Lang, Patrick \& Stritzke, 1998; Josephs \& Steele, 1990; Sayette, Martin, Perrott, Wertz \& Hufford, 2001). This phenomenon has led Steele \& Josephs (1988) to propose the attention-allocation model, which proposes that the ability of alcohol to decrease negative emotions, as well as its effects on behaviour in general, occur because of a narrowing of attentional capacity. This is argued to prevent intoxicated individuals from fully processing aversive stimuli, provided a more salient stimulus is also present, thus weakening their emotional response to those aversive stimuli (see also Sayette, 1993). Nevertheless, while the extent to which a reduction of negative emotion is a constant, as opposed to a context-dependent, feature of alcohol intoxication is disputed, it remains apparent that alcohol can reduce negative affect across a wide range of situations, and could therefore frequently diminish the emotional significance of errors.

\section{The Current Study}

Bartholow and colleagues (2012) provide a plausible account of how alcohol might interfere with the upregulation of cognitive control following errors: namely that alcohol reduces the negative affect elicited by errors, thereby weakening the motivation to correct 
them, or to avoid future errors. Existing research suggests that alcohol can attenuate emotional responses to aversive stimuli (e.g. Donohue et al., 2007; Levenson et al., 1980), that errors generate negative affect (e.g. Aarts et al., 2012; Elkins-Brown et al., 2017; Hajcak et al., 2004; Hajcak \& Foti, 2008), and that the effective use of cognitive control is at least partially dependent on the information provided by emotion (e.g. Frober et al., 2017; Inzlicht et al., 2015; Saunders et al., 2017). This evidence, combined with Bartholow and colleagues' observation that self-reported negative affect mediated the difference in post-error adjustment between their alcohol and control participants, lays out a convincing case in favour of their hypothesis.

However, the empirical data Bartholow and colleagues present in support of their model also suffer from a serious limitation. Their 2012 study does not actually test whether alcohol reduces the negative affect elicited by errors themselves, instead examining differences in participants' self-reported state negative affect prior to the task, then using this as a proxy for the effect of alcohol on their emotional responses to errors. We do not, therefore, have any direct evidence that alcohol makes errors less distressing, which raises an alternative explanation for Bartholow and colleagues' findings. Given that negativelyvalenced mood can facilitate cognitive control (Steenbergen, Band \& Hommel, 2010), alcohol intoxication might simply reduce overall state negative affect, thereby impairing cognitive control capabilities, without directly or dramatically changing the emotional processing of errors. This would not necessarily be at odds with the broader thesis that the regulation of cognitive control relies on negative affect. However, it is an issue if we are to assume, as Bartholow and colleagues do, that the effects of alcohol on error processing in experimental settings provide some insight into how and why alcohol affects social and cognitive functioning in the real world. If alcohol intoxication was merely acting as an unorthodox form of positive mood induction in these experiments, this would not explain 
why alcohol can have such deleterious effects on people's behaviour outside the lab; there are no laws against driving while in a good mood.

With this in mind, the aim of this study was to more rigorously test the hypothesis that alcohol reduces the negative affect elicited by errors, and thereby weakens or prevents the upregulation of cognitive control which normally follows an error. To do this I carried out two experiments in which I measured participants' emotional responses to errors in a task designed to require the use of cognitive control. My first experiment was a pilot study, in which I examined ANS responses to errors and post-error behavioural adaptation in a flanker task, in sober participants. I did this, firstly, to determine whether these phenomena were actually observable in the task I was using, before attempting to testing intoxicated participants, and secondly, to assess whether these ANS and behavioural measures were correlated with one another, as would be predicted by my hypothesis. In my second experiment participants completed the same task after consuming either an alcoholic or a placebo beverage, so that their ANS responses to errors and post-error cognitive adaptation could be compared, and my hypothesis thereby tested.

To measure participants' emotional responses to errors I recorded their heart rate (HR) and electrodermal activity while they performed a flanker task, then compared the degree of HR deceleration and skin conductance responses (SCR) observed following correct responses and errors on the task. These ANS responses are both highly sensitive to emotionally significant events (Bradley, 2009), and have been shown to be exaggerated following errors, especially in individuals high in trait negative affect (Hajcak et al., 2004). As discussed previously, a negative affective response to errors has been demonstrated using acoustic startle response (Hajcak \& Foti, 2008), facial electromyography (Elkins-Brown et al., 2017), and an evaluative priming task (Aarts et al., 2012). However, I opted to use HR and SCR to index negative affect instead, as acoustic startle and evaluative priming paradigms require 
additional task procedures after each trial, preventing measurement of changes in cognitive control immediately after errors. Measuring facial EMG, on the other hand, risks confounding changes in negative affect and changes in cognitive control; activity over the corrugator supercilii muscle, typically used to measure negative affect, may in fact be sensitive both variables (Hess, Philippot \& Blairy, 1998).

I chose to use a variant of the Eriksen flanker task (Eriksen \& Eriksen, 1974) to assess participants' use of cognitive control for several reasons. Firstly, while it may seem sensible to use the weapon identification task used by Bartholow and colleagues (2012), given that the goal of the study is primarily to extend their research, I was concerned that the racial biases that this task is predicated on might not generalise well outside of the USA. Secondly, the flanker task has frequently been used to study the effects of alcohol on error processing and cognitive control (e.g. Bailey et al., 2014; Bartholow et al., 2003; Nikolaou et al., 2013; Ridderinkhof et al., 2002), and so my use of this standard task allows me to relate my findings directly to the broader literature on cognitive control. Finally, to fully test my hypothesis it is important to have some way of measuring cognitive adaptation following errors. The flanker task is well-suited for this purpose, offering two behavioural indices of post-error adaptation: post-error slowing (PES) and post-error reduction of interference (PERI; Danielmeier \& Ullsperger, 2011).

To meet the specific needs of my study, I made several modifications to the flanker task used by Bailey and colleagues (2014). Firstly, I used letters, rather than arrows, as target stimuli. Because electrodermal activity was measured from the left hand, participants could only use their right hand to make task responses. Therefore, I wanted to avoid inadvertently biasing participants towards making "right arrow" responses. Secondly, I attempted to increase the difficulty of the task, so that participants would make a large number of errors, thereby minimizing the variance, and increasing the reliability, of measures recorded on error 
and post-error trials. I accomplished this by displaying letter stimuli very briefly $(75 \mathrm{~ms})$, against a low contrast background, and by giving participants a relatively short deadline to respond (800ms). Thirdly, the interval between trials was very long (up to 4s), so that SCRs from individual trials could be recorded. Finally, I added immediate feedback to the task, so that participants always knew whether they had answered correctly or incorrectly. It has previously been argued that alcohol makes it more difficult to accurately identify when an error has been made, and that this is why it impairs post-error adaptation (Ridderinkhof et al., 2002). Although empirical data suggest that intoxicated individuals are in fact capable of accurately judging their own performance (see Bartholow et al., 2012; Bailey et al., 2014), I nevertheless wanted to avoid any possible confound between error awareness and emotional responses to errors, and therefore included clear feedback after each trial.

In addition to recording participants' HR and skin conductance during the experiments, I also used electroencephalography (EEG) to monitor changes in participants' brain electrical potentials. In particular, I was interested in the error-related negativity (ERN), and how it might differ between intoxicated and sober participants. As previously elaborated, the ERN is an event-related potential which occurs following errors in speeded-response tasks, and is thought to reflect a motivationally-sensitive control signal generated within the anterior cingulate cortex (ACC). It is well established that alcohol diminishes the ERN (e.g. Bartholow et al., 2012; Ridderinkhof et al., 2002) Given that the ACC is assumed to constitute a critical component of the evaluative control system (Botvinick et al., 2011; Cavanagh \& Shackman, 2015), it is likely that this disruption of the ACC's response to errors is involved in any putative relationship between alcohol, emotion and post-error adaptation (Holroyd \& Yeung, 2003). Therefore, while testing my hypothesis did not depend directly on comparing the size of the ERN in intoxicated and sober participants, I nevertheless felt it was important, both as a way to confirm that I was investigating the same underlying 
phenomenon as previous studies, and to enable deeper exploration of the mechanisms which may support it.

\section{Experiment 1}

The aim of my first experiment was to pilot the flanker task on sober participants, to determine whether it would be appropriate for testing my hypothesis - that alcohol impairs post-error adaptation because of its reduction of the negative affect produced by errors - in a subsequent experiment involving alcohol. Assuming that the task used was in fact suitable for this purpose, I expected it to meet five criteria. Firstly, the task needed to be relatively difficult, so that participants, as a group, would make enough errors that their error responses could be reliably analysed. However, it was also important that the task was not so difficult as to cause participants to become disengaged and no longer care about making errors. Ideally, I wanted most subjects to make errors on at least $5 \%$, but not more than $25 \%$ of trials in the task.

Secondly, it was important that participants display a distinct ANS response to errors produced in the task, consistent with the premise that errors are experienced as aversive. Based on previous work by Hajcak, McDonald and Simons (2003b, 2004) I predicted that participants' SCRs would be larger and that they would show a greater degree of HR deceleration following errors than following correct responses.

Thirdly, I expected participants to show measurable behavioural adaptation following errors, ideally in the form of PERI, which could be used to infer an upregulation of cognitive control following errors. Although both PES and PERI have been linked to post-error recruitment of control processes (King et al., 2010), it has also been suggested that PES might actually reflect a general disruption of task performance due to orienting towards the error, rather than the implementation of cognitive control (Notebaert et al., 2009). PERI, on the other hand, is a relatively pure measure of post-error adaptation (Danielmeier \& Ullsperger, 
2011), and has previously been used to study the effects of alcohol on error processing (i.e. Bailey et al., 2014; Ridderinkhof et al., 2002). Nevertheless, I predicted that participants would demonstrate both PES - an overall increase in RT on trials following errors - and PERI - a decrease in the size of the flanker interference effect on post-error trials.

Fourthly, given the significance of the ACC in error processing and evaluative control, I expected participants to produce an ERN, characterized by a sharp decline in recorded EEG voltage at midfrontal sites immediately after error commission. Finally, my hypothesis implies that negative affect plays a causal role in driving adjustments in cognitive control following errors. Therefore, I expected to observe correlations between indices of negative affect (SCR and HR change following errors) and behavioural measures of post-error adaptation (PES and PERI). Bartholow and colleagues (2012) made effective use of mediation analysis in examining a relationship between negative affect and post-error adaptation, and I hoped to be able to use a similar procedure in the second experiment to test my hypothesis. Significant correlations between dependent variables measuring emotional response to errors and variables measuring post-error adaptation would be a prerequisite for carrying out such an analysis.

\section{Method}

\section{Participants}

Twenty-eight participants (19 female, 8 male, 1 other) between the age of 18 and $50(M$ $=21.57, S D=7.10)$ were recruited. Participants reported having normal or corrected-to normal vision, and that they were not receiving treatment (including psychotherapy) for depression, ADHD, or an anxiety disorder. I chose to exclude participants suffering from these particular disorders as they are all known to substantially alter error processing (O’Connell et al., 2004; Olvet \& Hajcak, 2008). Participants were compensated for their time 
with either course credit or movie vouchers. The study was approved by the Human Ethics Committee of the School of Psychology, Victoria University of Wellington.

One participant's data was removed from all analyses due to low accuracy $(<.66)$ in the flanker task. All of the remaining 27 participants completed the flanker task, while behavioural performance and ANS measures were recorded, as described below. For a subset of 16 participants, EEG recordings were also collected during the task.

\section{The Letter Flanker Task}

Participants completed a computer-based flanker task consisting of trials in which strings of five letters were presented on the screen in a horizontal line. See figure 1 for a visual representation of the trial procedure. All letters in the string were either a capital ' $\mathrm{H}$ ' or a capital ' $\mathrm{S}$ ' (Courier New font). Participants were required to identify the central letter of the string by pressing either the ' 1 ' or the ' 2 ' key, located on the number pad of the keyboard, with the index and middle fingers of their right hands, respectively. Assignment of response keys was counterbalanced across participants. On $50 \%$ of trials, the four peripheral flanker letters were identical to the central letter (congruent trials), on the other 50\% they were different; i.e. if the central letter was an ' $\mathrm{H}$ ', each of the flanker letters would be an ' $\mathrm{S}$ ' and vice versa (incongruent trials).

Letter stimuli were approximately $.5^{\circ} \times .7^{\circ}$ visual angle each, with the entire string subtending roughly $2.7^{\circ} \times .7^{\circ}$ visual angle. To increase the potential for flanker interference, and to ensure a relatively large number of task errors, the horizontal position of the letter string varied between trials: the central letter in the string could either be located exactly in the centre of the screen, or it could be offset either $.55^{\circ}$ or $1.10^{\circ}$ visual angle to the left, or to the right, of the centre. To further promote flanker interference, the flanker letters were printed in a darker shade of grey than central letters (RGB 60, 60, 60 for the outermost letters, RGB 80, 80, 80 for the inner flanker letters, RGB 100, 100,100 for central letters; see 
Ridderinkhof et al., 2002). Letters were presented on a light grey background (RGB 140, 140, 140).

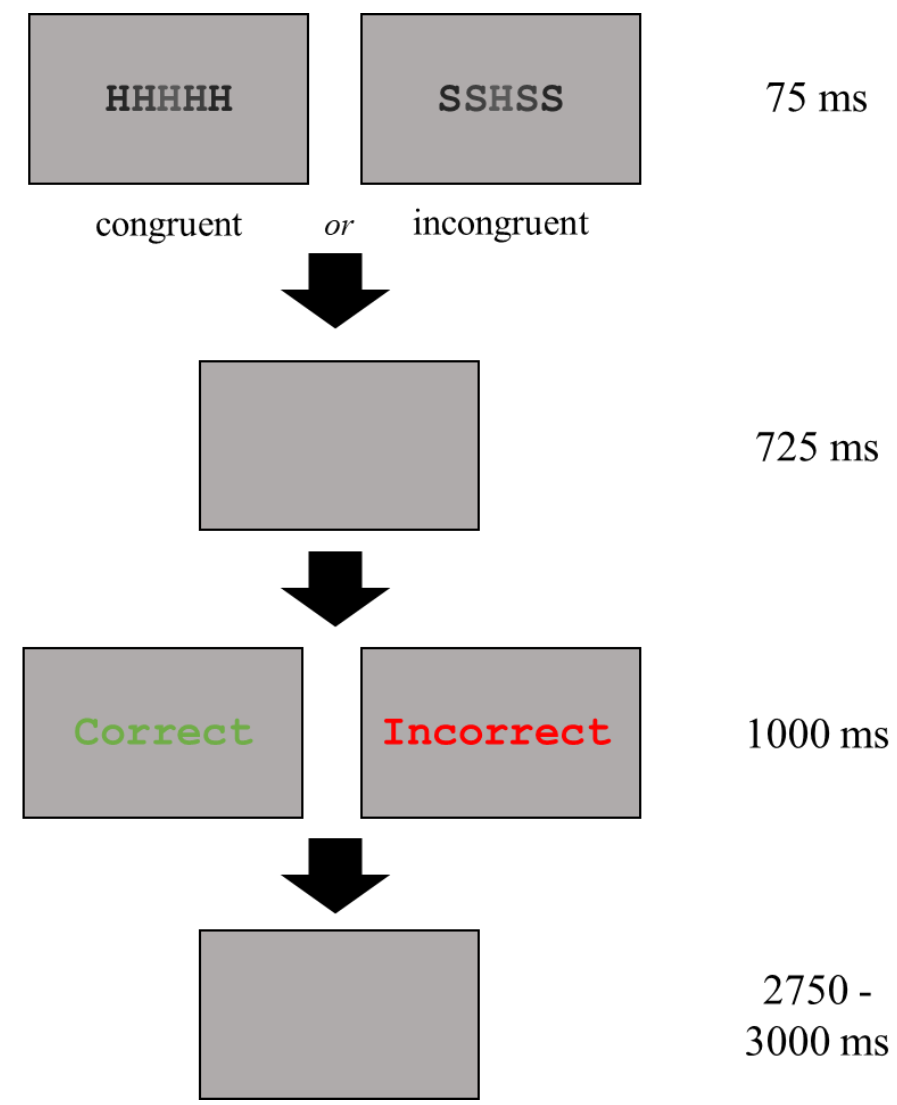

Figure 1. Schematic of trial procedure in the letter flanker task. Note that letter and feedback stimuli are not displayed to scale.

Letter strings were presented for $75 \mathrm{~ms}$. Following stimulus presentation, participants had an additional $725 \mathrm{~ms}$ to identify the central letter (or $800 \mathrm{~ms}$ in total). After pressing a key, participants immediately received feedback for their response: the word 'Correct' printed in green, or the word 'Incorrect' printed in red, if they pressed the wrong key. If participants did not press either the ' 1 ' or the ' 2 ' key within $800 \mathrm{~ms}$ of a letter string appearing, the words 'Please respond faster' appeared on the screen, printed in red. Feedback was presented for 1000ms. Following feedback, the screen stayed blank for between 2750 and 3000ms, after which a new trial began with the presentation of a new letter string. The task was made up of 
two short practice blocks of 10 trials each, followed by 20 experimental blocks each containing 20 trials.

The task was created and run using E-Prime version 2.0 (Psychology Software Tools, Pittsburgh USA). Stimuli were presented using an ASUS VG248QE monitor with a resolution of 1920 x 1080 and a vertical refresh rate of $144 \mathrm{~Hz}$. A chin rest was used to maintain a constant viewing distance of $57.3 \mathrm{~cm}$.

\section{ANS Recording and Analysis}

Skin conductance was monitored using a pair of dry stainless-steel electrodes, attached to the medial phalanges of the left index and ring fingers, and connected to an ADInstruments ML116 AC GSR Amp. Signals were digitized at $1 \mathrm{kHz}$ using ADInstrument's Powerlab 16/30 and recorded in LabChart 8.0.1. Because skin conductance is a relatively slow response (Boucsein, Fowles, Grimnes, Ben-Shakhar, Roth, Dawson \& Filion, 2012), it was typically not feasible to identify discrete SCR peaks in the interval between a response and the next trial. Following Hajcak and colleagues (2003b), I therefore operationalized SCR as the maximum value, in micro-Siemens ( $\mu S)$, occurring between .5 and 3 seconds following a response, relative to the mean value in the .5 seconds prior to that response. Responses smaller than $0.02 \mu \mathrm{S}$ were scored as zero. Because the distribution of SCRs is often highly skewed (Lykken \& Venables, 1971), raw SCR values on each trial were logarithmically transformed, using the formula $\mathrm{SCR}=\ln ($ raw $\mathrm{SCR}+1)$, to normalize the data.

Heart rate was measured using electrocardiography (ECG). The signal was acquired via disposable adhesive $\mathrm{Ag} / \mathrm{AgCl}$ electrodes placed on the right shoulder and lower left ribcage, referenced to an electrode on the left shoulder, and amplified using an ADInstruments ML138 Octal Bio Amp. The signal was then digitized at 1kHz using ADInstrument's Powerlab 16/30, recorded in LabChart 8.0.1, and band-pass filtered offline 
between 1 and $400 \mathrm{~Hz}$. Heart rate in beats per minute (BPM), was determined via the interval between successive R-wave spikes, defined as peaks greater than 2 standard deviations above mean ECG activity. The 3 seconds following each task response were divided into twelve $500 \mathrm{~ms}$ epochs, and heart rate within each epoch, relative to heart rate in the $500 \mathrm{~ms}$ prior to that response, was calculated.

\section{Electrophysiological Recording and Analysis}

EEG recordings were made using a Brain Products actiCAP active electrode system. Five $\mathrm{Ag} / \mathrm{AgCl}$ electrodes were placed at scalp sites corresponding to $\mathrm{Fz}, \mathrm{FCz}, \mathrm{Cz}, \mathrm{Pz}$ and $\mathrm{Oz}$ in the international 10-20 system (American Electroencephalographic Society, 1994). I chose to record only from midline sites, as I did not intend to precisely localize event-related potentials, and was interested primarily in the ERN and other forms of error-related brain responses, which are known to be observed primarily at frontal and central sites along the midline (Cavanagh et al., 2012; Gehring et al., 1993).Vertical electrooculogram (EOG) was recorded using electrodes placed above and below the left eye, while horizontal EOG was recorded using electrodes placed on the outer canthus of each eye. Electrodes were also placed on mastoid bones behind each ear, with online recordings referenced to the left mastoid. Electrode impedances were kept below $20 \mathrm{k} \Omega$; compared to passive electrodes, active electrode systems produce minimal noise at moderate impedance levels (Mathewson, Harrison \& Kizuk, 2017). Impedances were checked periodically during the experiment and adjusted if necessary. The EEG signal was amplified with a Brain Products actiCHamp amplifier and digitized at $500 \mathrm{~Hz}$ with Brain-Vision Recorder. Data was filtered online with a high-pass filter of $0.02 \mathrm{~Hz}$.

EEG recordings were analysed in Brain-vision Analyzer 2.0. Recordings were rereferenced offline to the average of the right and left mastoids, band-pass filtered from 0.01 and $30 \mathrm{~Hz}$, using a zero phase-shift Butterworth filter $(12 \mathrm{~dB} / \mathrm{oct})$, and notch filtered at $50 \mathrm{~Hz}$. 
To analyse error-related brain potentials, recordings were segmented into $650 \mathrm{~ms}$ epochs, beginning $200 \mathrm{~ms}$ before, and ending 450ms after, a task response. Segments were baseline corrected by subtracting the mean activity in the $200 \mathrm{~ms}$ prior to a response. Segments were then corrected for ocular artifacts using Gratton and Coles' method (Gratton, Coles \& Donchin, 1983). While artifact removal using independent components analysis is often preferred to regression-based methods, such as Gratton and Coles', as it minimizes the amount of genuine brain activity removed along with ocular artifacts (Wallstrom, Kass, Miller, Cohn \& Fox., 2004), the small number of recording sites used in this experiment made using ICA impractical (Jung, Humphries, Lee, Makeig, McKeown, Iragui \& Sejnowski, 1998). Segments were re-baselined after ocular correction. Segments containing recording artifacts, defined as voltages outside the range of -150 to $150 \mu \mathrm{V}$, at the primary electrodes of interest (Fz, $\mathrm{FCz}$ and $\mathrm{Cz})$, were removed.

The ERN is typically characterized as a negative deflection at midfrontal sites, peaking between 50 and 80ms after an error (Gehring et al., 1993). Therefore, the ERN was quantified as the mean negative peak voltage occurring at the FCz electrode within 100ms of a response, as is typical within the literature (e.g. Cavanagh et al., 2012; Ridderinkhof et al., 2002).

\section{Procedure}

Participants completed the experiment individually, with each session taking approximately an hour and 15 minutes. After giving written consent, participants were fitted with electrodes needed to record heart rate, skin conductance and EEG. Participants then completed the letter flanker task, after which they were debriefed on the purpose of the study.

\section{Design}

The experiment used a within-subjects experimental design. To analyse participants' overall performance on the task, flanker congruency (congruency vs. incongruent) was used 
as an independent variable, with accuracy and response time acting and dependent variables. To analyse physiological and behavioural responses to errors, response type (correct response vs. incorrect response), although not directly manipulated functioned as the primary independent variable. Primary dependent variables in these analyses were skin conductance response $(\mu \mathrm{S})$, change in heart rate (BPM) and ERN amplitude $(\mu \mathrm{V})$, and response time (RT; to assess PES) and flanker interference (RT on incongruent trials - RT on congruent trials; to assess PERI) on the following trial.

\section{Results}

\section{Behavioural Measures}

Mean accuracy (the percentage of correct responses) in the task was $83.9 \%$ ( $S D=$ $6.9 \%)$, with participants failing to respond on $3.4 \%(S D=2.3 \%)$ of trials and making errors on $12.8 \%(S D=6.7 \%)$ of trials. Of the 27 participants analysed, 24 had an error of between 5 and $25 \%$ ( 1 participant $>25 \%, 2$ participants $<5 \%$ ), suggesting that the task was appropriately difficult for the purpose of analysing the emotional impact of errors. Participants were approximately twice as likely to make errors on trials on which incongruent stimuli were presented, compared to trials on which congruent stimuli were presented see figure 2). A paired-samples t-test indicated that this difference was statistically significant $\left(t(26)=4.643, p<.001, d_{\mathrm{z}}=.893\right)$. To assess the effect of flanker interference on RT, mean RTs on correct trials were calculated for congruent and incongruent trials separately (see figure 3). A paired samples t-test found that mean RTs were significantly greater on incongruent trials than congruent trials $\left(t(26)=16.685, p<.001, d_{\mathrm{z}}=3.211\right)$. These results indicate that incongruent flanker stimuli elicited response conflict, decreasing both speed and accuracy, thereby establishing the task as a meaningful test of participants' cognitive control abilities. 


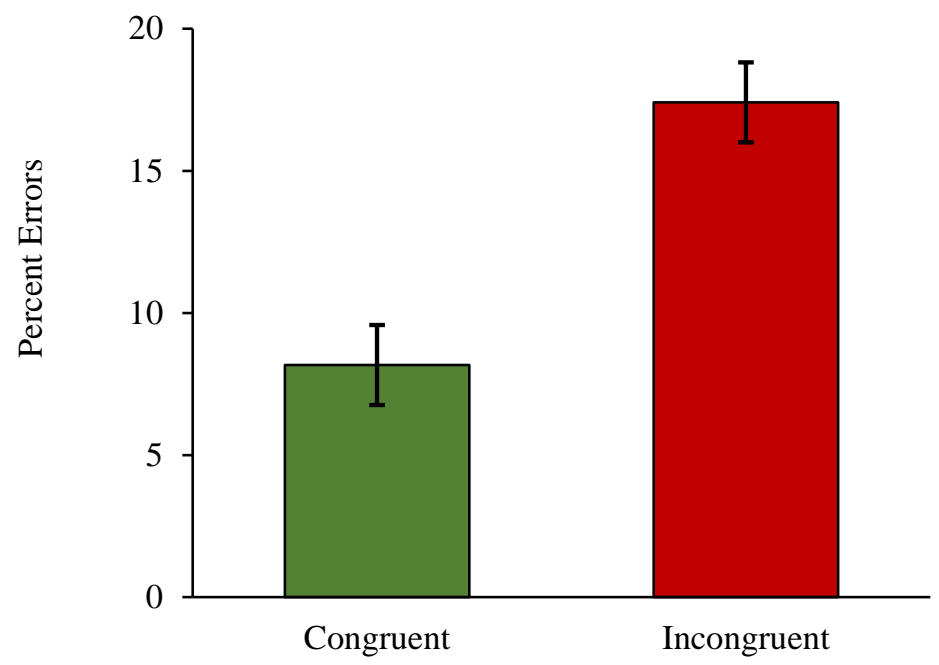

Figure 2. Mean percentage of error responses in the flanker task to congruent and incongruent flanker stimuli. Error bars show the standard error of the mean, normalized and corrected for a within-subjects design (Morey, 2008).

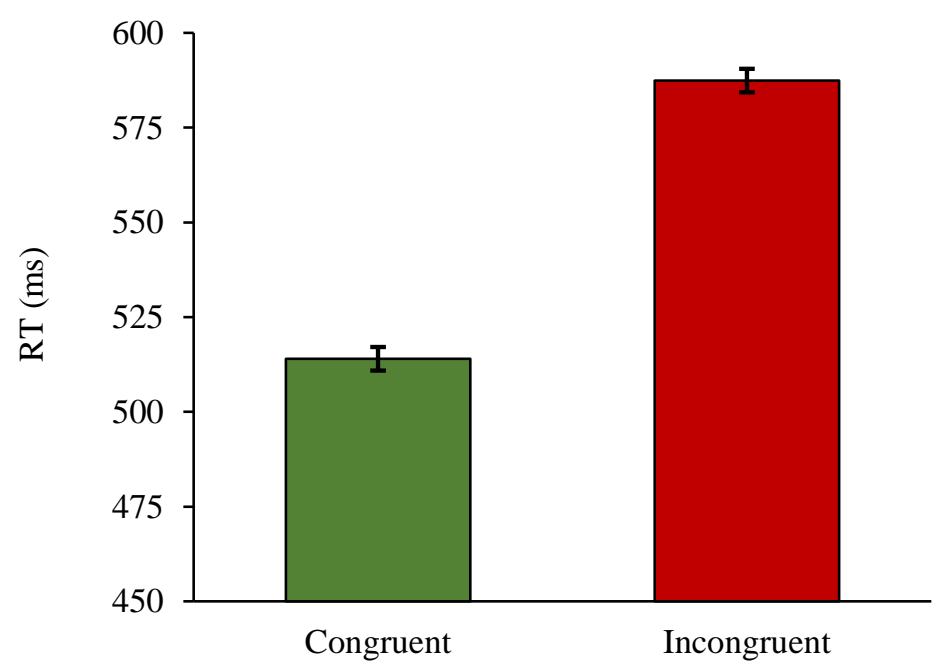

Figure 3. Mean RT (ms) in the flanker task for correct responses to congruent and incongruent flanker stimuli. Error bars show the standard error of the mean, normalized and corrected for a within-subjects design (Morey, 2008).

Evidence for behavioural adaption following task errors was limited. Post-error slowing (PES), defined as an increase in mean RT on correct responses following errors, relative to 
correct responses following correct responses, was modest, but statistically significant, as demonstrated in a paired samples t-test $\left(t(26)=2.948, p=.007, d_{\mathrm{z}}=.567\right.$, see figure 4$)$. However, no such evidence was found for post-error reduction of interference (PERI), defined as a decrease in flanker interference (RT incongruent - RT congruent, correct responses only) on trials following errors, compared to trials following correct responses. ( $p$ $>$.1, see figure 5). My prediction that participants would upregulate cognitive control following errors was, therefore, only weakly supported.

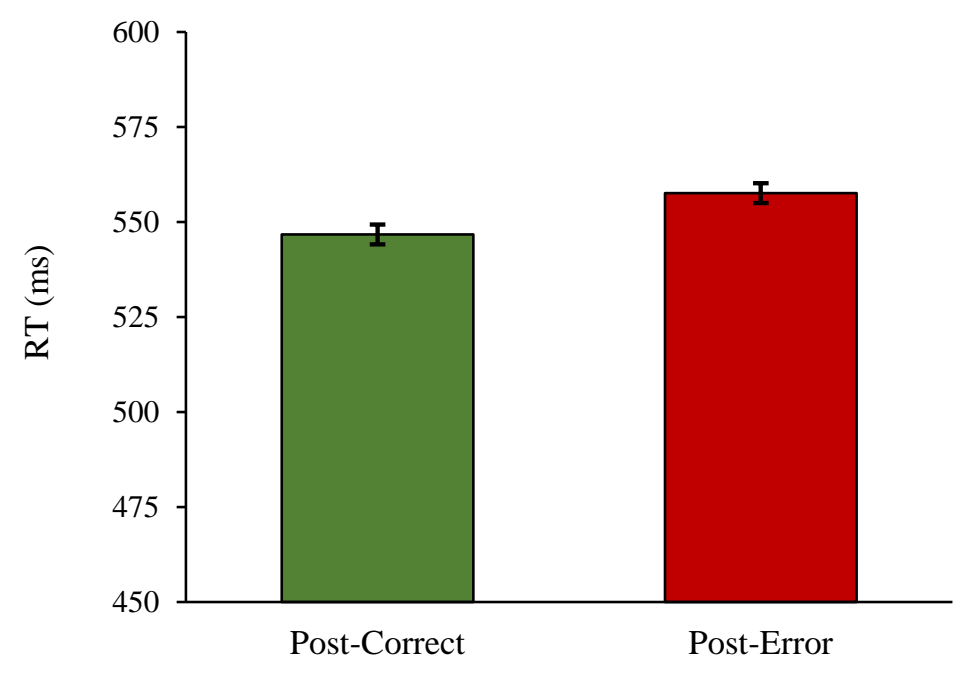

Figure 4. Mean RT (ms) in the flanker task for correct responses, following a correct response or an error on the previous trial. Error bars show the standard error of the mean, normalized and corrected for a within-subjects design (Morey, 2008).

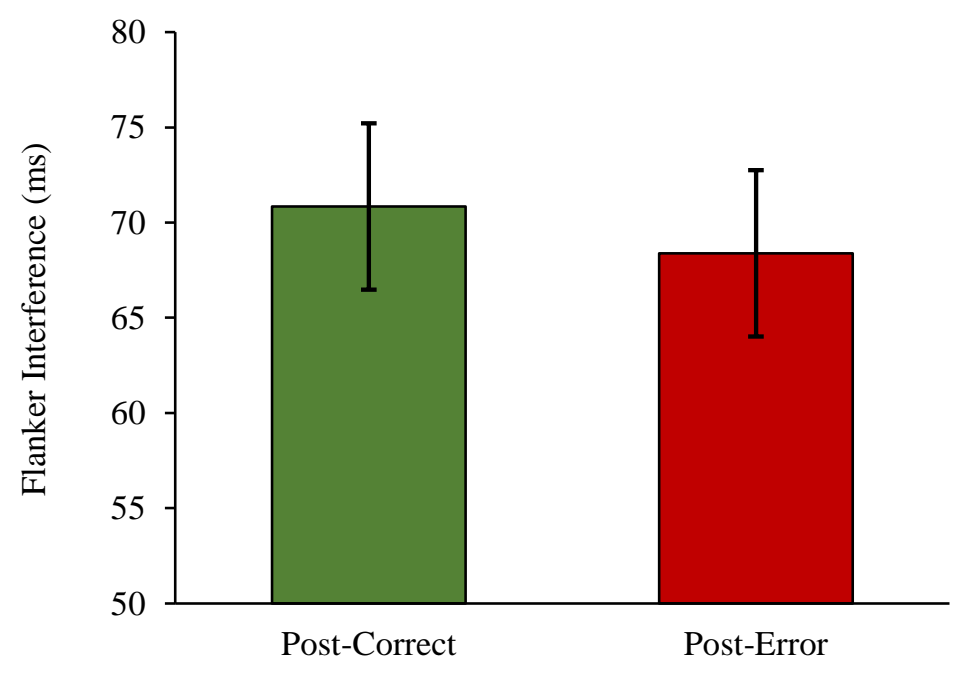


Figure 5. Size of flanker interference effect (RT incongruent-RT congruent) for correct responses, following a correct response or an error on the previous trial. Decreased flanker interference following an error is indicative of PERI. Error bars show the standard error of the mean, normalized and corrected for a within-subjects design (Morey, 2008).

\section{ANS Measures}

Figure 6 displays the SCRs observed following errors and correct responses. Although SCRs to errors appeared to be substantially larger than SCRs to correct responses, as was predicted, a paired samples t-test found that the difference between the two means did not reach the threshold of statistical significance $\left(t(26)=1.925, p=.065, d_{\mathrm{z}}=.371\right)$.

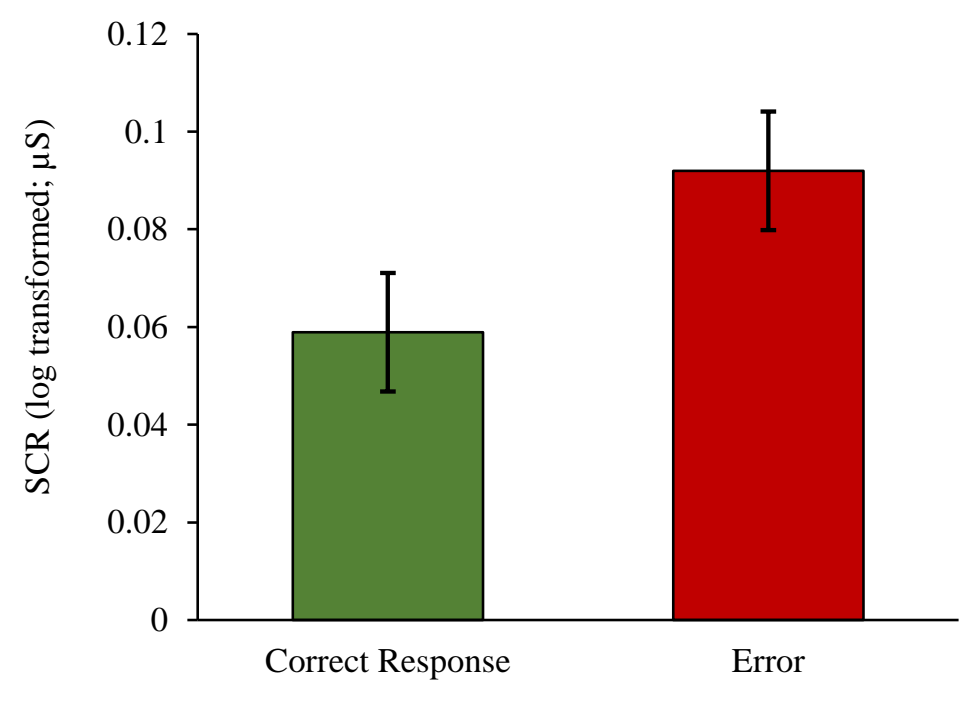

Figure 6. Mean log-transformed skin conductance responses $(\mu S)$ made following correct responses and errors. Error bars show the standard error of the mean, normalized and corrected for a within-subjects design (Morey, 2008).

Figure 7 shows the average change in HR at each time interval measured, following errors and correct responses, across all participants. The overall pattern of the data was similar to that previously observed by Hajcak and colleagues (2003b, 2004): HR responses were characterized by a fast initial deceleration, followed by a more sustained acceleration, with the deceleration component being substantially larger following errors than correct 
responses. A 2 (response type) x 6 (time) repeated measures ANOVA demonstrated a significant main effect of response type $\left(F(1,26)=22.368, p<.001, \eta_{\mathrm{p}}{ }^{2}=.462\right)$, supporting the conclusion that errors produced greater HR deceleration than correct responses. A main effect of time was also found $\left(F(1.323,34.401)=46.024, p<.001, \eta_{\mathrm{p}}{ }^{2}=.639\right.$, GreenhouseGeisser correction applied due to violation of sphericity), consistent with a shift from HR deceleration to acceleration over the $3 \mathrm{~s}$ period. The interaction between response type and time did not reach the threshold of statistical significance, $(F(1.743,45.314)=3.139, p=$ $.059, \eta_{\mathrm{p}}^{2}=.108$, Greenhouse-Geisser correction applied due to violation of sphericity), suggesting that the extent of the difference in HR change between correct responses and errors remained at least relatively constant over the 3 s period.

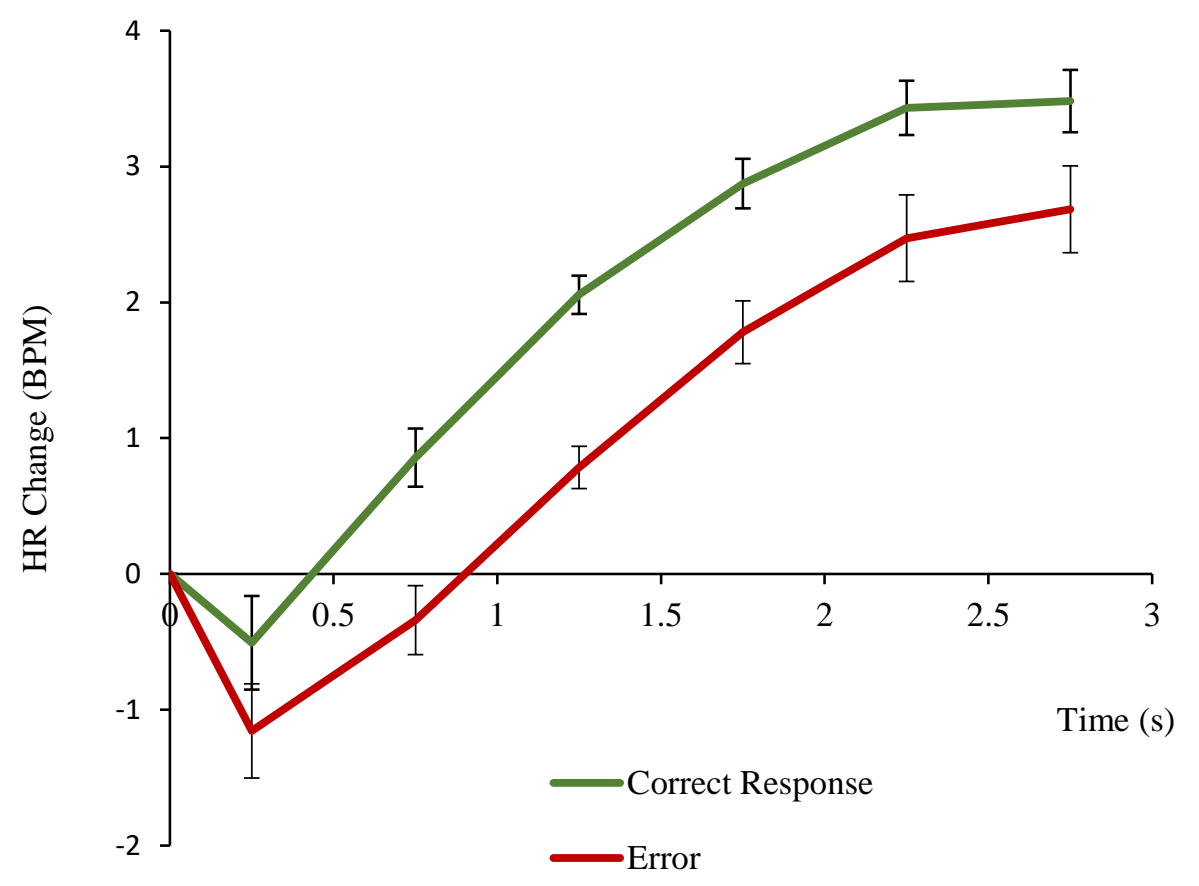

Figure 7. Mean change in heart rate (BPM) from pre-response baseline, over the 3 s following correct responses and errors. Error bars show the standard error of the mean, normalized and corrected for a within-subjects design (Morey, 2008). 
Although the difference in SCR between correct responses and errors was not statistically significant $(p=.059)$, the overall trend of the ANS data was in line with the prediction that participants would display physiological responses consistent with negative affect, following errors. However, given that participants were far more likely to make errors when presented with incongruent stimuli, I was concerned that any apparent differences in ANS responses between errors and correct responses might be confounded by ANS responses to incongruent stimuli. There is considerable evidence that response conflict itself generates negative affect (Frober et al., 2017; Saunders et al., 2017; Steenbergen, Band \& Hommel, 2009). High-conflict incongruent letter strings could therefore plausibly elicit a measurable ANS response. To determine whether this was potentially influencing my findings, I compared SCR and HR change following errors and correct responses made on incongruent trials only. If the differences in ANS responses previously observed between correct and incorrect trials were to disappear under these conditions, this would suggest that they were actually artifacts caused by differing proportions of congruent and incongruent trials in the two response conditions.

Results of the analysis can be seen in figure 8. For both ANS variables the overall pattern (i.e. greater SCRs and HR deceleration to incorrect responses) of the results were very similar to those obtained when both congruent and incongruent trials were used. Furthermore, repeating the t-test used to analyse SCR using only data from incongruent trials, indicated that SCR following errors was in fact larger than SCR following correct responses under these constraints, in this case at a .05 level of significance $\left(t(26)=2.276, p=.031, d_{\mathrm{z}}=.438\right)$. Additionally, repeating the ANOVA used to analyse HR using only data from incongruent trials produced the same main effect of response type $\left(F(1,26)=42.120, p<.001, \eta_{\mathrm{p}}{ }^{2}=\right.$ .618). These analyses indicate that any ANS responses to conflict occurring during stimulus 
presentation are not large or enduring enough to confound observed ANS responses to errors, when both congruent and incongruent trials are included.
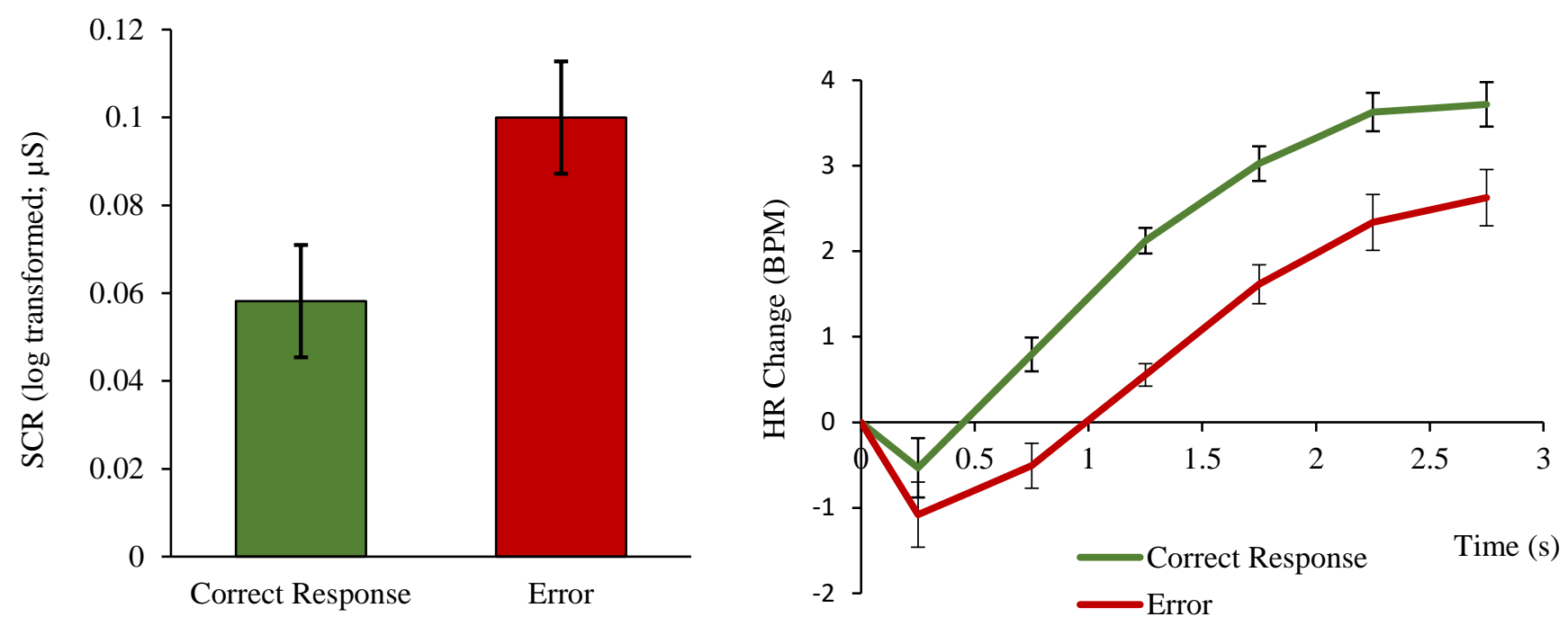

Figure 8. Graphs of SCR (left) and HR (right) responses following correct and erroneous responses made to incongruent stimuli only. Error bars show the standard error of the means, normalized and corrected for within-subjects designs (Morey, 2008).

\section{Electrophysiological Measures}

Figure 9 shows response-locked ERPs recorded at the FCz electrode within a 650ms epoch, spanning from $200 \mathrm{~ms}$ before, to $450 \mathrm{~ms}$ after, correct responses and errors. As predicted, a large negative deflection was observed following errors, peaking approximately $60 \mathrm{~ms}$ after an incorrect response. The presence of an ERN was confirmed through a paired samples t-test, which found a significantly larger peak negative voltage 100ms after an error, in comparison to a correct response $\left(t(15)=6.727, p<.001, d_{\mathrm{z}}=1.681\right)$. 


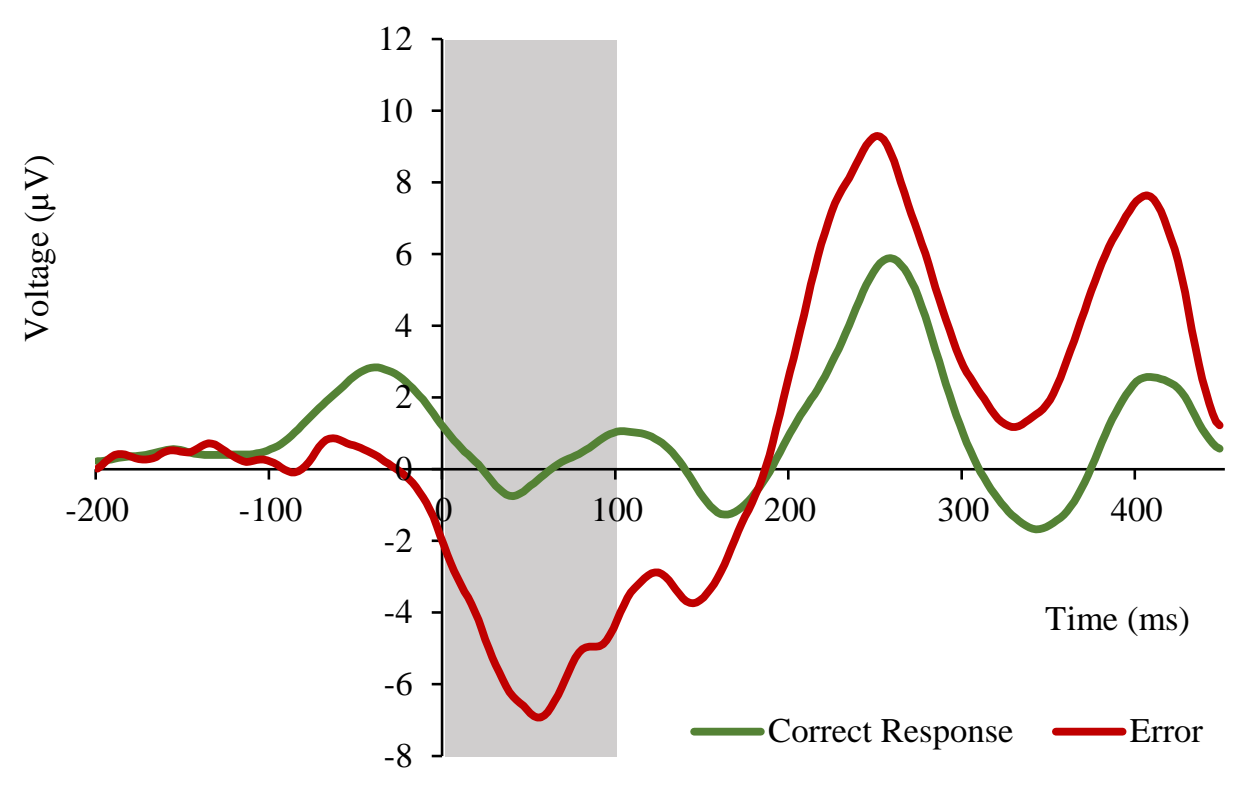

Figure 9. Event related potentials elicited by correct responses and errors, at the $F C z$ electrode. The shaded area represents the time window in which the ERN was identified.

Visual examination of midline electrode activity also revealed greater positivity from around $200 \mathrm{~ms}$ after an error, in comparison to a correct response, consistent with the presence of the error positivity (Pe) ERP component. The negative deflection occurring around 300ms after both erroneous and correct responses, may be due to a FRN elicited by the feedback participants received immediately after their responses (see Castellar, Kühn, Fias \& Notebaert, 2010).

For comparative purposes, figure 10 shows the mean voltage recorded over the same period at the $\mathrm{Fz}$ and $\mathrm{Cz}$ electrodes. ERPs observed at these electrodes were very similar, on the whole, to those observed at FCz, although the ERN peak was at its greatest at FCz. Furthermore, scalp mapping of the difference between errors and correct responses (see figure 11) indicated that the ERN was most prominent at the $\mathrm{FCz}$ and $\mathrm{Cz}$ electrodes. We can 
therefore conclude that it is reasonable to follow previous studies (e.g. Ridderinkhof et al., 2002) in assuming activity at FCz to be representative of ACC response to errors.
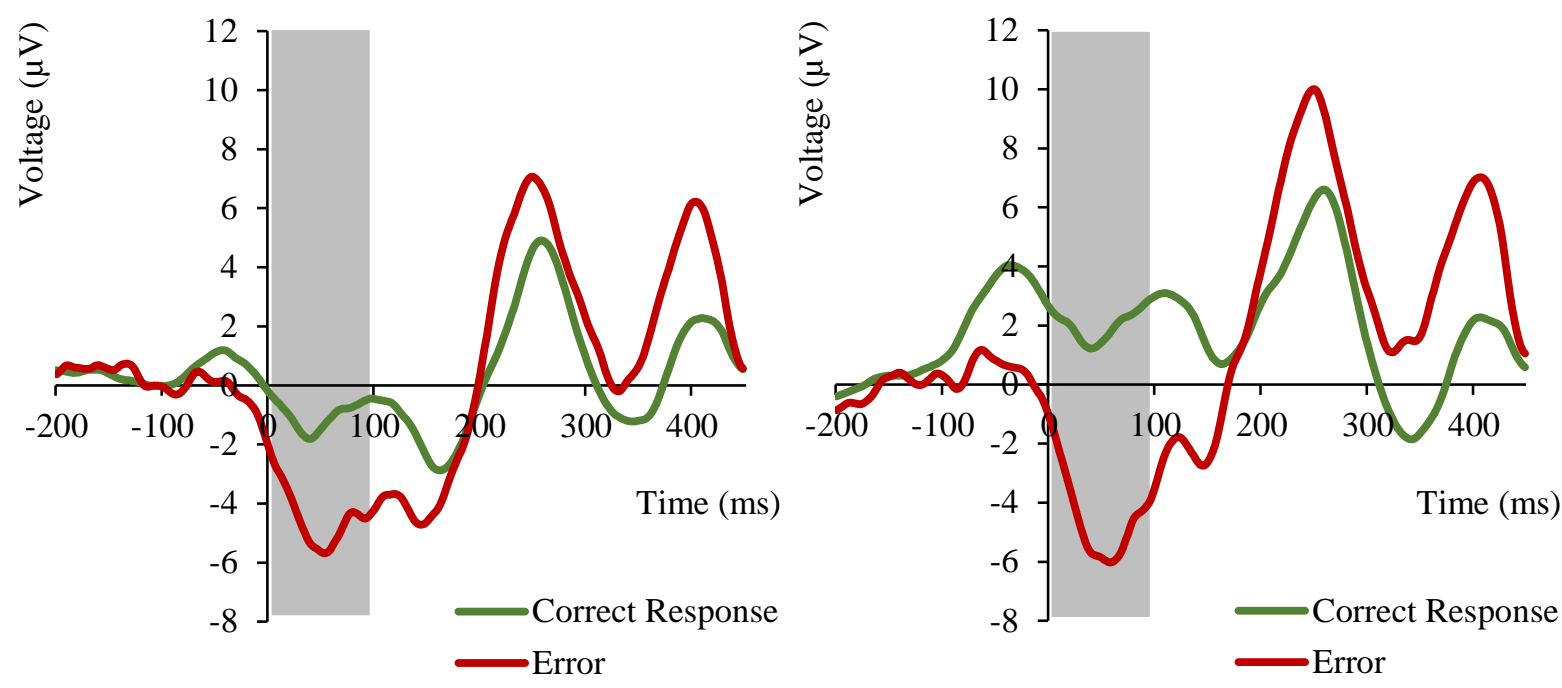

Figure 10. Event related potentials elicited by correct responses and errors, at the Fz (left) and $C z$ (right) electrodes. The shaded areas represents the time windows in which the ERN were identified.

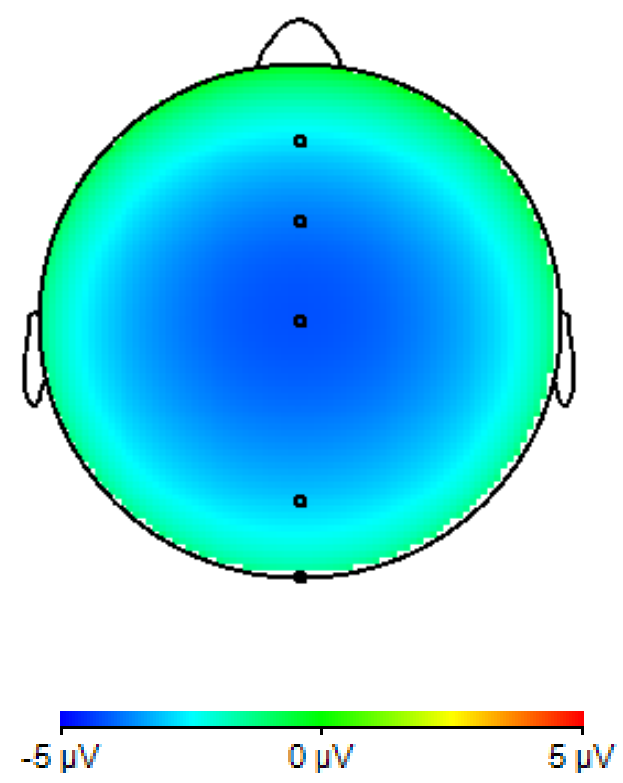

Figure 11. Scalp distributions of voltage difference between errors and correct responses, in the 100ms period following a response. 
However, as with participants' ANS responses, I was concerned that errorrelated ERPs might be confounded with ERPs elicited by high-conflict incongruent stimuli. Response conflict is known to produce a midline ERP qualitatively similar to the ERN, the anterior N2 (see Carter et al., 1998; Cavanagh et at., 2012). Figure 12 shows ERPs made following correct responses and errors to incongruent stimuli only. Consistent with the ANS data, including only responses to incongruent trials did not seem change the overall pattern of the data in any meaningful way. A paired-samples t-test comparing ERN peaks from only incongruent trials found that voltage was still significantly larger after an error than a correct response $\left(t(15)=6.025, p<.001, d_{\mathrm{z}}=\right.$ 1.506), indicating that it was acceptable to include data from both congruent and incongruent trials in statistical analyses.

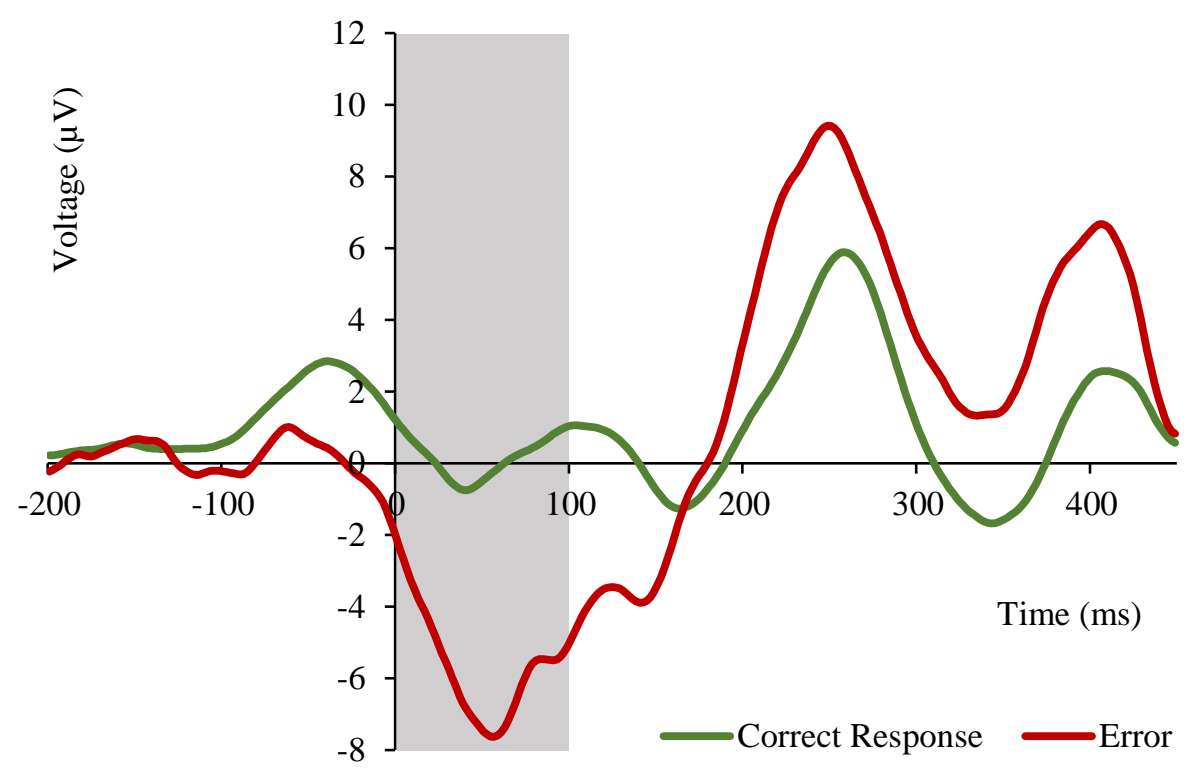

Figure 12. Event related potentials elicited by correct responses and errors made to incongruent stimuli only, at the FCz electrode. The shaded area represents the time window in which the ERN was identified. 


\section{Correlational Data}

Table 1. Correlations between psychophysiological measures, and post-error reduction of interference and post-error slowing.

\begin{tabular}{|c|c|c|c|c|}
\hline & SCR error & ERN FCz & PES & PERI \\
\hline $\begin{array}{l}\Delta \mathrm{HR} 0-.5 \mathrm{~s} \\
\text { error }\end{array}$ & -.290 & -.371 & .018 & -.242 \\
\hline $\begin{array}{l}\Delta \mathrm{HR} .5-1 \mathrm{~s} \\
\quad \text { error }\end{array}$ & -.207 & -.187 & -.085 & -.314 \\
\hline $\begin{array}{l}\Delta \mathrm{HR} 1-1.5 \mathrm{~s} \\
\quad \text { error }\end{array}$ & -.170 & -.011 & -.137 & $-.418 *$ \\
\hline $\begin{array}{l}\Delta \mathrm{HR} 1.5-2 \mathrm{~s} \\
\quad \text { error }\end{array}$ & -.157 & .047 & -.097 & $-.499 * *$ \\
\hline $\begin{array}{l}\Delta \mathrm{HR} 2-2.5 \mathrm{~s} \\
\quad \text { error }\end{array}$ & -.180 & .061 & -.028 & $-.519 * *$ \\
\hline $\begin{array}{c}\Delta \mathrm{HR} 2.5-3 \mathrm{~s} \\
\quad \text { error }\end{array}$ & -.172 & .081 & .017 & $-.493 * *$ \\
\hline SCR error & & $-.496 \#$ & -.067 & $.496 * *$ \\
\hline ERN FCz & & & $-.463 \#$ & $-.481 \#$ \\
\hline PES & & & & .140 \\
\hline
\end{tabular}

To determine whether participants' affective responses to errors predicted their ability to cognitively adapt after those errors, I computed bivariate correlations between physiological measures sensitive to the motivational significance of errors (SCR to errors, HR change following errors, the ERN), and behavioural measures indexing post-error adaptation (PES and PERI). Results are presented in table 1. A significant positive correlation was found between participants' (log-transformed) SCR to errors and the degree of PERI they displayed. A significant negative correlation was also found between HR change from 1s after an error onwards and PERI (i.e. greater HR deceleration after an error was associated 
with better control of flanker interference on trials following errors). However, neither SCR nor HR change following errors predicted PES. The magnitude of the ERN at FCz was negatively associated with both PERI, PES and SCR magnitude (i.e. a more negative shift in voltage following errors predicted better post-error adaptation, and larger SCRs), albeit not more than marginally significantly. Overall, correlational data was consistent with the prediction that participants who demonstrated a stronger physiological response to errors would also show superior post-error adaptation, supporting the idea that increased cognitive control following error commission is driven by the negative affect generated by errors.

\section{Discussion}

The aim of Experiment 1 was to determine whether the flanker task I had designed could be used to assess the emotional impact of errors in intoxicated participants. On the whole, results indicated that it was well suited for this purpose, with four of the five previously outlined criteria for an appropriate task being fully satisfied, and one being partially satisfied. The first criterion, that the task would have an appropriate level of difficulty, was met, with the majority of participants' (24 out of 27 , approximately $89 \%$ ) error rates lying between 5 and 25\%. Assuming, based on Bailey and colleagues' (2014) findings, that alcohol would not drastically increase participants error rate, this proportion of errors indicated that the task was likely to elicit enough errors in the second experiment for reliable analysis, while not being so difficult as to produce frustration or disengagement.

The second criterion, that participants would show ANS responses consistent with negative affect upon making errors, was also largely achieved. Although the difference in SCR magnitude following correct responses and errors did not meet the threshold for statistical significance, the direction of the effect was consistent with previous studies (i.e. Hajcak et al., 2003b, 2004). Moreover, a significant effect was found when only incongruent trials were considered. I therefore felt confident that SCR could still be a useful index of 
emotional processing in the second experiment, provided that statistical power was increased. Furthermore, as predicted, a robust increase in HR deceleration was observed following errors, compared to correct responses, thus validating change in HR as a useful index of affective processing within this task.

The fourth criterion, that participants would display an ERN, was satisfied, with substantial negative deflections in EEG voltage observed immediately following errors but not correct responses. This demonstrates that errors elicited an ACC response from participants, as would be expected based on previous research and theory. Additionally, participants also showed EEG activity consistent with the presence of an FRN, raising the possibility of including this as a supplementary index of ACC response to errors in the second experiment.

The fifth criterion, that physiological indices of the negative affect elicited by errors and measures of post-error adaptation would be correlated, was also met. While PES did not correlate with SCR or HR change following errors, PERI was moderately correlated with both ANS measures, implying that negative affect was playing a role in driving adjustments in participants' cognitive control settings within the task, as would be predicted by my overall hypothesis. This suggests that, in addition to being suitable for testing whether alcohol alters the emotional impact of errors, my task was also capable of investigating whether alcohol's effects on cognitive control are in fact driven by these changes. This would potentially be accomplished through a mediation analysis, like the one used by Bartholow and colleagues (2012).

Unfortunately, the third criterion, that participants would show behavioural measures of post-error adaptation, was only partially satisfied. While participants did display a limited degree of PES, there was no evidence for PERI among the group as a whole. This may have been because the long response-stimulus interval meant that short-term adjustments in 
cognitive control following errors faded before the next trial could begin. However, the robust correlation between PERI and HR and SCR suggests that some participants, i.e. those displaying a stronger emotional reaction to error commission, did successfully reduce flanker interference after an error, while other participants' interference may have actually increased. Bailey and colleagues (2014) found that while their control participants displayed similar levels of flanker interference after errors and correct responses, participants receiving alcohol showed greater interference after errors. They argued that cognitive control is likely at its lowest just prior to an error, and that alcohol prevented control from being expediently recovered. With this in mind, I concluded that PERI might still provide a meaningful measure of post-error adaptation on my task. Overall, results from the first experiment indicated that the flanker task I had designed was well-suited for investigating the effects of alcohol on the emotional significance of errors. Therefore, I decided not to make any modifications to the task before beginning my second experiment.

\section{Experiment 2}

The aim of the second experiment was to rigorously test the hypothesis that alcohol reduces the negative affect elicited by errors, and that this leads to an impairment in the increased engagement of cognitive control which normally follows an error. To this end, the flanker task used in the first experiment was administered to two new groups of participants: one which received a moderate dose of alcohol prior to testing, and a placebo group, which instead received sham alcohol. I chose to use a placebo group as a control condition to account for any potential expectancy effects of alcohol, which can be significant in cognitive control tasks (Testa et al., 2006). While an experiment including a third condition, in which participants received neither alcohol nor placebo, would arguably have been preferable for this purpose, this was not feasible given the time and resources available. 
Several new dependent measures were also incorporated into the study, in addition to those recorded in the original experiment. Firstly, following Bartholow and colleagues (2012), participants' state negative affect was measured using the PANAS, upon arrival in the lab and again after beverage administration, with the aim of replicating their finding that alcohol reduced participants' levels of negative affect, and that this mediated alcohol's effects on post-error adaptation. Secondly, having observed ERP activity consistent with an FRN in the first experiment, I decided to investigate whether this component differed in alcohol and placebo group participants. I expected that the FRN, like the ERN, would be attenuated in intoxicated subjects. However, to the best of my knowledge, no previous studies have investigated the effect of alcohol on the FRN, and it is unknown whether alcohol affects the processing of external cues signalling errors, indexed by the FRN, in same way that it affects the processing of internally generated error signals. I was concerned, however, that any apparent differences in FRN magnitude would be difficult to disentangle from potential alcohol effects on other, simultaneously occurring ERP components, namely the error positivity and the P3 (see Castellar et al., 2010). Therefore, I also carried out a timefrequency analysis on the EEG data, via the complex wavelet technique, in order to track changes in midfrontal theta following task responses. Midfrontal theta reflects activity in the ACC, the underlying source of both the ERN and the FRN (Cavanagh et al., 2012). Measuring midfrontal theta from 100ms after an error onwards, after the time window in which the ERN is typically observed, should therefore reflect the ACC control response produced by error feedback. Incorporating time-frequency analysis of EEG data also enabled an additional measure of post-error adaptation, in the form of occipital alpha suppression. As discussed previously, occipital alpha power is indicative of selective attention and task engagement (Foxe et al., 1998), and a decline in alpha power following an error likely 
reflects increased engagement of attention as the result of upregulated control (Carp \& Compton, 2009).

Based on my hypothesis, I had four main predictions regarding the outcome of Experiment 2. Firstly, I predicted that participants in the alcohol group would have a diminished emotional response to errors on the flanker task, demonstrated through smaller SCRs, and decreased HR deceleration following errors, compared to participants in the placebo group. Secondly, I predicted that the alcohol group would display reduced postadaptation, and that this would be reflected by less PERI in the alcohol group compared to the placebo group, possibly to the point of flanker interference actually increasing after errors, as observed by Bailey and colleagues (2014), as well as lower levels of PES and posterror alpha suppression. Thirdly, I predicted that alcohol group participants would produce smaller ERNs than placebo group participants. Finally, I predicted that differences in posterror adaptation between the alcohol and placebo groups would be mediated by group differences in SCR and HR responses to errors.

\section{Method}

\section{Participants}

73 moderate drinkers between the age of 18 and 30 participated in the experiment in exchange for movie vouchers. I had intended to collect data from 80 participants (see preregistration) but was forced to terminate data collection due to time constraints. Potential participants were excluded if they reported consuming fewer than 3 or more than 25 standard alcoholic drinks per week, if they were pregnant or breastfeeding, if they had a history of drug or alcohol dependence, if they were taking any psychiatric medications, or if they were diagnosed with a medical condition or were taking medications which could contraindicate alcohol use. All participants also reported that they had normal or corrected-to-normal vision, and that they were not currently receiving treatment (including psychotherapy) for 
depression, ADHD, or an anxiety disorder. The study was approved by the Human Ethics Committee of the School of Psychology, Victoria University of Wellington.

Participants were randomly assigned to receive either alcohol or placebo. Three participants' data were removed from all analyses due to low accuracy $(<.66)$ in the flanker task, leaving 36 participants in the alcohol group (mean age $=23.25, S D=5.38,23$ female, 13 male) and 34 in the placebo group (mean age $=22.74, S D=3.94,20$ female, 14 male). Additionally, one alcohol group participant was excluded from ERP and time-frequency analyses only due to excessive EEG recording artifacts.

\section{Beverage Administration}

Participants assigned to the alcohol group consumed an alcoholic beverage composed of 2 parts 75-proof vodka to 3 parts each tonic water and orange juice. For participants in the placebo condition, the vodka was replaced with a mixture containing 5\% vodka and $95 \%$, tonic water, poured from a vodka bottle. Participants in both groups were told that the beverage was intended make them "moderately drunk". If they inquired further, they were informed that the beverage was meant to bring their blood alcohol concentration (BAC) to $0.08 \%$, and told that this was the legal limit for driving in New Zealand prior to 2014.

The quantity of vodka given to alcohol group participants was in fact expected to produce a peak BAC of $0.08 \%$. The volume needed was estimated for each participant based on their body weight, using the Widmark formula (Posey and Mozayani, 2007). R factor values used in the calculation were .73 for males and .66 for females. This formula resulted in alcohol group participants receiving a mean dose of $127 \mathrm{ml}$ vodka $(S D=23)$, equivalent to approximately 3.8 standard drinks. Participants in the placebo condition were given a volume of sham vodka calculated using the same formula, thereby producing an estimated peak BAC of $0.004 \%$. 
In both conditions, beverages were mixed in view of participants, and then divided evenly between three glasses. Participants were then given 5 minutes to consume the contents of each glass. To facilitate the deception in the placebo condition, the rim of each glass was smeared with vodka using a facecloth before the participant entered the lab.

\section{The Positive and Negative Affect Scale}

Participants' state levels of positive and negative affect were assessed upon arrival in the lab, and approximately 10 minutes after beverage administration, using the Positive and Negative Affect Scale (PANAS; Watson, Clark, \& Tellegen, 1988). The PANAS is a short 20 item self-report scale, in which respondents rate on a scale from 1 (lowest) to 5 (highest), the extent to which they feel, either currently, or in general, twenty different feelings or emotions. Ten of these emotions are positive (e.g. excited, proud) and ten are negative (e.g. nervous, irritable), yielding two averaged scores, for positive and negative affect respectively, that range from 1 to 5 (see Appendix A). The PANAS has been shown to have good internal consistency and external validity (Crawford \& Henry, 2004). In this experiment, participants were asked to indicate their current feelings.

\section{The Letter Flanker Task}

Participants completed the same letter flanker task used in Experiment 1. However, the task was divided into two parts, so that participants could complete the practice trials before consuming alcohol, and the experimental trials after. In all other respects the task was identical to the one used in the first experiment.

\section{ANS Recording and Analysis}

Participants' electrodermal activity and HR were recorded during the experiment, and processed to obtain values for analysis, using the exact same procedures as in Experiment 1. Considering that analysing only responses on incongruent trials produced larger error effects in my first experiment, a case could have been made for excluding data from congruent trials. 
However, I felt that including more trials should, in principle, give more reliable results, given that I had no theoretical rationale for considering errors on incongruent trials as more representative of the underlying emotional response I was trying to operationalize.

Furthermore, using both types of trials allowed my ANS measures to remain consistent with my behavioural and ERP measures of error processing.

\section{Electrophysiological Recording and Analysis}

EEG recording during the experiment and processing of the ERN followed procedures identical to those used in Experiment 1. However, several additional exploratory analyses were also performed on participants' EEG data. Firstly, to analyse the FRN, raw EEG data was processed using the same procedure as with the ERN, except that, for each participant, the FRN was quantified as the difference between the mean peak positive voltage and the mean peak negative voltage, occurring between 200 and 400ms post-response.

Time-frequency analyses of midfrontal theta and occipital alpha power were, like ERP analyses, carried out using Brain-vision Analyzer 2.0. Raw EEG recordings were first rereferenced offline to the average of the right and left mastoids, then filtered $(0.01-30 \mathrm{~Hz}$ band-pass, $50 \mathrm{~Hz}$ notch). Data was then divided into 5000ms segments, beginning $2500 \mathrm{~ms}$ before, and ending 2500ms after, each task response. Segments were corrected for ocular artifacts using Gratton and Coles' method (1983), and then removed if they reached a voltage of less than $-150 \mu \mathrm{V}$ or greater than $150 \mu \mathrm{V}$ at the FCz or Oz electrodes. EEG power $\left(\mu \mathrm{V}^{2}\right)$ within each remaining segment was estimated using a continuous complex Morlet wavelet transformation consisting of 20 logarithmically spaced steps, ranging from 1 to $20 \mathrm{~Hz}$. This generated 5 layers within the theta frequency band (central frequencies of 4.1, 4.8, 5.7, 6.6 and $7.8 \mathrm{~Hz}$ ) and 3 layers within the alpha frequency band (central frequencies of 9.1, 10.6 and $12.5 \mathrm{~Hz})$. 
To analyse post-response theta power, power estimates at $\mathrm{FCz}$, within each layer in the theta band, were averaged over the period from 100 to $500 \mathrm{~ms}$ following a response. Participants' occipital alpha power was assessed at four different time points in order to track changes in cognitive control over the course of a trial. Alpha power at Oz was averaged across each layer in the alpha band between 1000 and $800 \mathrm{~ms}$ before a response, immediately before the presentation of a flanker display; between 200 and 0ms before a response, when participants were in the process of selecting a response; between 500 and 700ms after a response, while participants were viewing feedback; and between 1800 and 2000ms after a response, during the response-stimulus interval.

\section{Procedure}

Participants completed the experiment individually, with each session taking approximately an hour and 45 minutes to complete. Participants were asked to refrain from drinking alcohol or using illicit drugs for 24 hours prior to the experiment, and to avoid undertaking strenuous exercise or drinking caffeinated beverages for 3 hours prior. After giving written consent, participants indicated their current affective state using the PANAS, and were then introduced to the letter flanker task, completing the 20 practice trials. Set-up of the EEG and heart rate electrodes was then partially completed, after which beverages were mixed and consumed within 15 minutes. After drinking, participants waited for ten minutes, allowing time to finish setting up the recording equipment, and for alcohol to be absorbed in the alcohol condition.

Participants then completed the PANAS for a second time and were then reminded of the instructions for the letter flanker task. Before beginning the flanker task, participants were asked to rate their subjective level of intoxication on a scale from 1 (not drunk at all) to 10 (the most drunk I've ever been), and their breath alcohol concentration was measured using an Alco-Scan AL9000 breathalyser. Participants then completed the flanker task. Breath 
alcohol concentrations were assessed again halfway through, and upon completion of the task. After finishing, participants were debriefed on the purpose of the study, and their condition was revealed to them. Participants were required to stay in the lab until their BAC (estimated via breathalyser) fell below $0.02 \%$.

\section{Design}

The experiment used a mixed experimental design. Independent variables were beverage group (between-subjects; alcohol vs. placebo) and congruency (within-subjects; congruent vs. incongruent). Primary dependent variables were change in self-reported negative affect on the PANAS after beverage administration and response time. When analysing participants' responses to errors, independent variables were beverage group (between-subjects; alcohol vs. placebo) and response type (within-subjects; correct response vs. incorrect response), while dependent measures were skin conductance response $(\mu S)$, change in heart rate (BPM), and ERN amplitude $(\mu \mathrm{V})$, and RT and flanker interference (RT on incongruent trials - RT on congruent trials) on the following trial (used to calculate PES and PERI, respectively).

\section{Preregistration}

The hypotheses and predictions of my second experiment, and an outline of the design and planned analyses, were registered through the Open Science Framework prior to data collection. My preregistration can be viewed at https://osf.io/u6m2k/ (also see Appendix B).

\section{Results}

\section{Manipulation Checks}

Measurements taken via breathalyser indicated that alcohol group participants had a mean BAC of $0.037 \%(S D=.012)$ before beginning the flanker task, a mean BAC of $0.038 \%$ $(S D=.010)$ halfway through the flanker task, and a mean BAC of $0.036 \%(S D=.008)$ after finishing the flanker task. These readings were consistently much lower than expected, and 
likely reflect poor calibration of the breathalyser used, given that the Widmark formula used to estimate alcohol dosages is widely used and has been well validated (Posey and Mozayani, 2007). All participants in the placebo group were recorded as having a BAC of 0 at all three time points.

Mean rating of subjective intoxication before beginning the task was $4.38(S D=1.14)$ in the alcohol group and $2.63(S D=1.29)$ in the placebo group. An independent samples ttest found that alcohol group participants felt significantly more intoxicated than placebo group participants $(t(68)=5.999, p<.001, d=1.456)$. However, a one sample t-test also found that placebo group participants' ratings were significantly greater than $1(t(33)=7.396$, $p<.001, d=1.268)$, indicating that the deception was at least somewhat effective; as a group, participants in the placebo condition did believe they had consumed alcohol and were feeling its effects.

\section{Behavioural Measures}

As can be seen in table 2, there was remarkably little difference in the mean proportion of correct responses, errors and failures to respond in the flanker task, between the alcohol and placebo groups. An independent samples t-test found no significant difference in error rate between conditions $(p>.1)$, confirming that alcohol group participants were no more likely to make errors in the flanker task than placebo group participants.

Figure 13 displays mean RTs to congruent and incongruent target displays, for correct responses only, within each condition. RT data was entered into a 2 (stimulus type) x 2 (beverage group) mixed ANOVA. A significant interaction was observed between stimulus type and beverage group $\left(F(1,68)=4.014, p=.049, \eta_{\mathrm{p}}^{2}=.056\right)$. Follow-up paired samples $\mathrm{t}$ tests found that RTs were significantly greater on incongruent trials than congruent trials in both the placebo $\left(t(33)=18.971, p<.001, d_{z}=3.253\right)$ and alcohol groups $(t(35)=20.967, p$ $\left.<.001, d_{\mathrm{z}}=3.495\right)$, indicating that a substantial flanker interference effect occurred in both 
conditions. However, the interaction observed in the ANOVA indicates interference was significantly greater in the alcohol group $(M=71.1, S D=20.4)$ than in the placebo group $(M$ $=61.7, S D=19.0)$. This suggests that alcohol intoxication may have led to a general impairment in participants' ability to exercise cognitive control.

Table 2. Mean (SD) percentage of each response type (correct response, error or slow response) within each condition.

\begin{tabular}{|c|ccc|}
\hline Beverage Group & Correct Responses (\%) & Errors (\%) & Failures to Respond (\%) \\
\hline Placebo & $86.5(7.4)$ & $10.6(6.6)$ & $2.9(2.0)$ \\
Alcohol & $86.3(5.93)$ & $10.7(4.4)$ & $3(2.5)$ \\
\hline
\end{tabular}

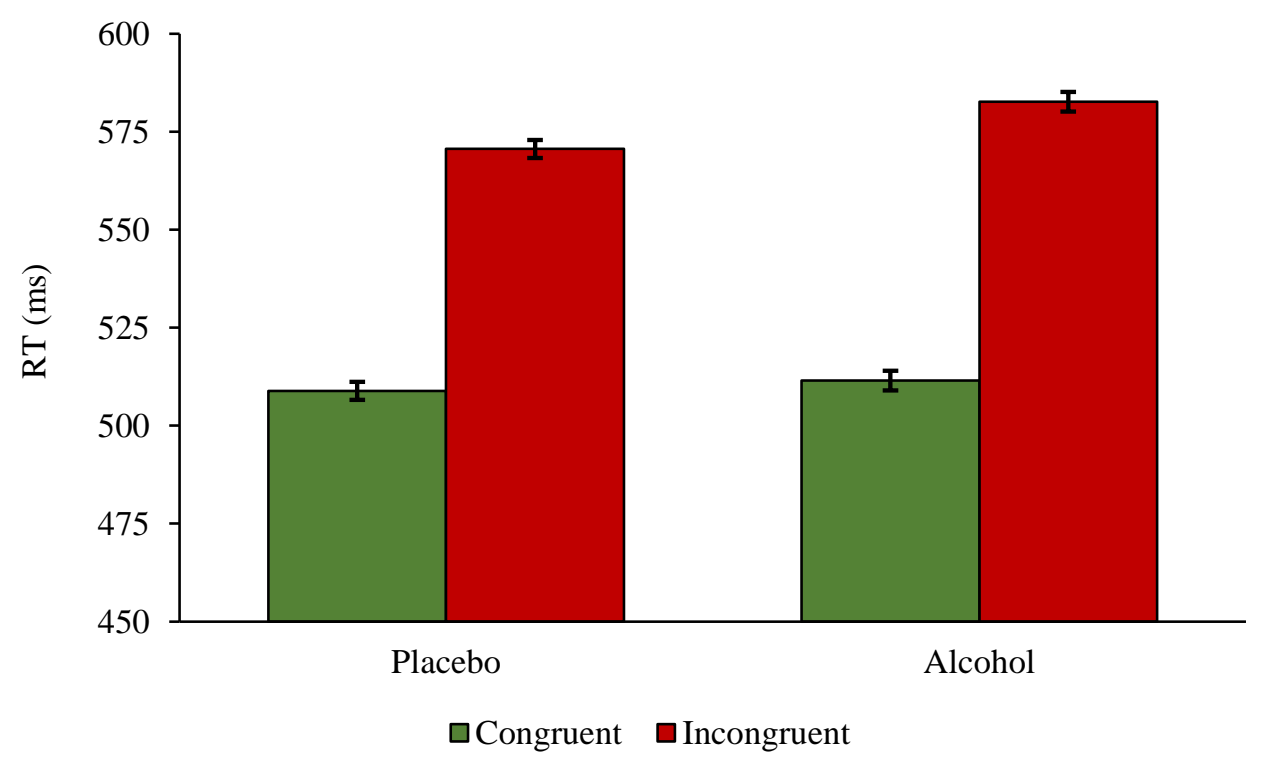

Figure 13. Mean RT (ms) in the flanker task, for correct responses to congruent and incongruent stimuli, within each condition. Error bars show the standard error of the mean, normalized and corrected for a within-subjects design (Morey, 2008).

Post-error slowing (PES) was observed in both conditions (see figure 14), with a 2 (previous response type) x 2 (beverage group) mixed ANOVA finding a significant main effect of previous response type on $\mathrm{RT}\left(F(1,68)=38.607, p<.001, \eta_{\mathrm{p}}{ }^{2}=.362\right)$. However, 
there was no significant interaction between previous response type and beverage group ( $p>$ .1 ), indicating that PES was not reduced in the alcohol group, as might be expected if cognitive adaptation following errors were impaired by alcohol. There was no main effect of beverage group $(p>.1)$.

Neither group showed any evidence of post-error reduction of interference (PERI; see figure 15). A 2 (previous response type) x 2 (beverage group) mixed ANOVA did not find a main effect of previous response type on flanker interference $(p>.1)$, or any interaction between previous response type and beverage group $(p>.1)$. A significant main effect of beverage group was found $\left(F(1,68)=4.078, p=.047, \eta_{\mathrm{p}}^{2}=.057\right)$, consistent with a general impairment of cognitive control in the alcohol condition. However, given that neither PES nor PERI was diminished in alcohol group participants, the prediction that alcohol would interfere specifically with behavioural measures of post-error adaptation, was not supported by the data.

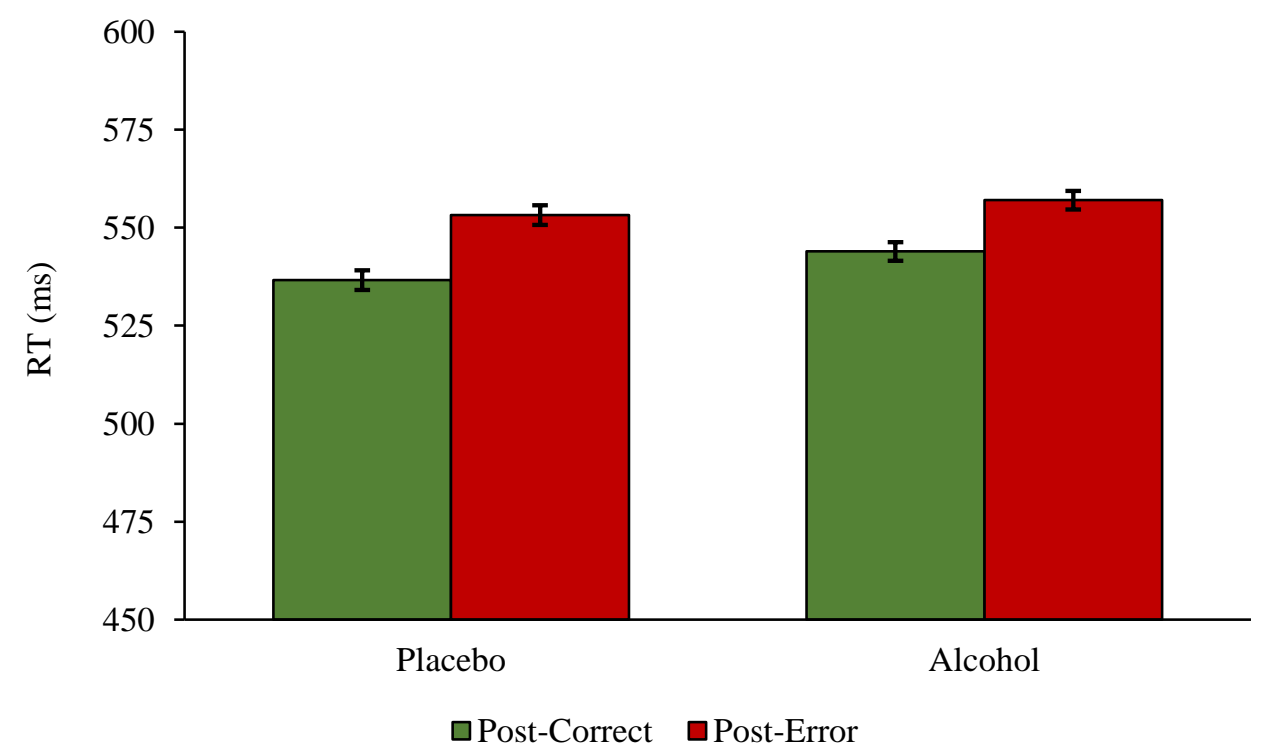

Figure 14. Mean RT (ms) in the flanker task for correct responses, following a correct response or an error on the previous trial, within each condition. Error bars show the standard error of the mean, normalized and corrected for a within-subjects design (Morey, 2008). 


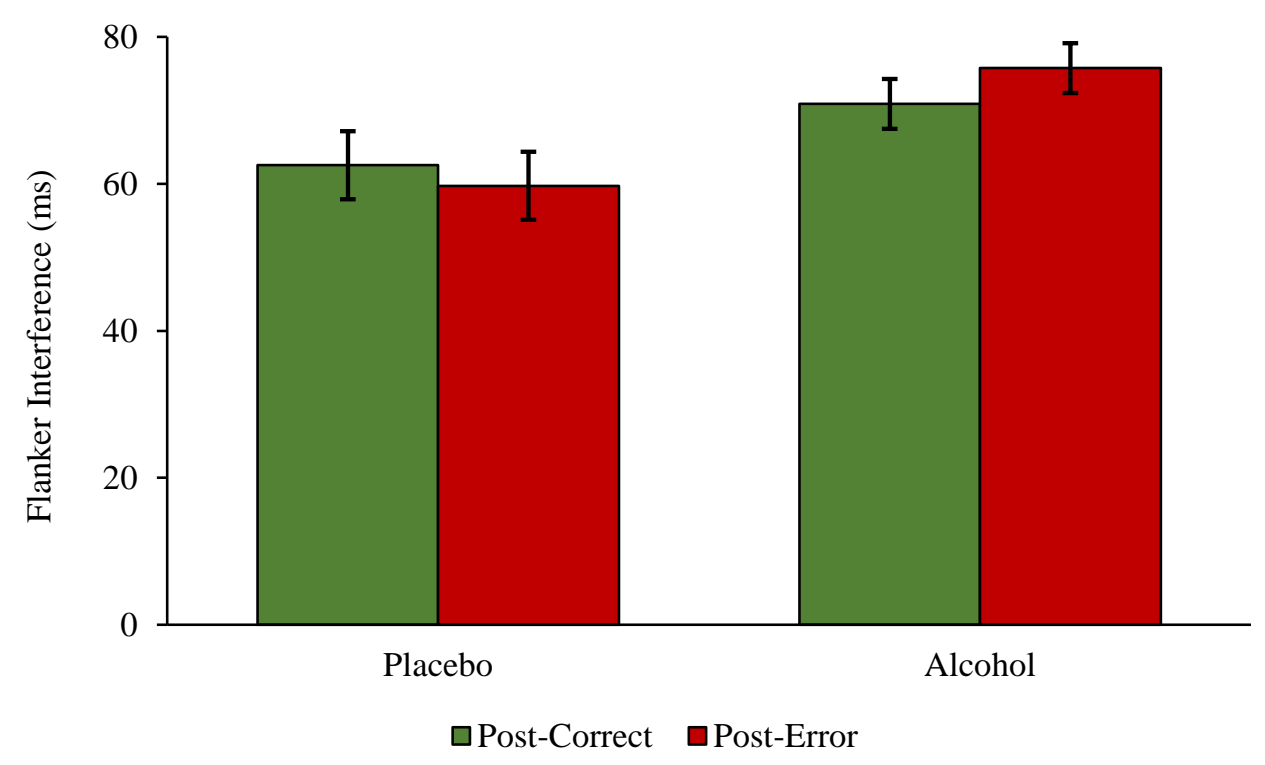

Figure 15. Size of flanker interference effect ( $R T$ incongruent - RT congruent), for correct responses, following a correct response or an error on the previous trial, within each condition. Decreased flanker interference following an error is indicative of PERI. Error bars show the standard error of the mean, normalized and corrected for a within-subjects design (Morey, 2008).

\section{PANAS Data}

PANAS ratings of participants within each beverage group are displayed in table 3 . In contrast to Bartholow and colleagues' findings (2012), participants in the alcohol and placebo groups displayed a similar level of overall negative affect after beverage consumption. A 2 (time) $\mathrm{x} 2$ (beverage group) mixed ANOVA found a main effect of time $(F(1,68)=19.697, p$ $<.001, \eta_{\mathrm{p}}^{2}=.225$ ), indicating that subjects in both groups experienced less negative affect after drinking compared to baseline. However, there was no main effect of beverage group ( $p$ $>.1)$, nor any significant interaction between time and beverage group $(p>.1)$.

In regard to participants' self-reported positive affect, a 2 (time) x 2 (beverage group) mixed ANOVA revealed an interaction between time and beverage group $(F(1,68)=4.899$, $\left.p=.030, \eta_{\mathrm{p}}{ }^{2}=.067\right)$. Follow up paired samples t-tests demonstrated that, relative to baseline, 
positive affect declined significantly following beverage consumption in the placebo group $\left(t(33)=3.712, p=.001, d_{z}=0.637\right)$, but not in the alcohol group $(p>.1)$, suggesting that alcohol consumption may have counteracted a decline in positive affect normally occurring between participants' arrival in the lab and the beginning of the experiment.

Table 3. Mean $(S D)$ ratings for positive and negative affect items on the PANAS, upon entering the lab and after beverage administration, for participants in each condition.

\begin{tabular}{|c|ccc|ccc|}
\hline \multirow{2}{*}{$\begin{array}{c}\text { Beverage } \\
\text { Group }\end{array}$} & \multicolumn{3}{|c|}{ Negative Affect } & \multicolumn{3}{c|}{ Positive Affect } \\
\cline { 2 - 7 } & Baseline & Post-Beverage & Change & Baseline & Post-Beverage & Change \\
\hline Placebo & $1.46(.40)$ & $1.24(.28)$ & $-0.21(.38)$ & $3.27(.56)$ & $2.99(.78)$ & $-0.28(.44)$ \\
Alcohol & $1.32(.27)$ & $1.21(.26)$ & $-0.11(.22)$ & $3.15(.70)$ & $3.09(.68)$ & $-0.06(.40)$ \\
\hline
\end{tabular}

\section{ANS Measures}

Mean SCRs to errors and correct responses within each condition are displayed in figure 16. SCR data was entered into a 2 (response type) x 2 (beverage group) mixed ANOVA. A significant main effect of response type was found $(F(1,68)=14.757, p<.001$, $\eta_{\mathrm{p}}^{2}=.178$ ), indicating that, within both groups, errors elicited larger SCRs than correct responses. Contrary to my predictions, SCRs to errors were not attenuated in the alcohol group. Although overall SCR magnitude appears to be exaggerated in the alcohol group, the lack of any significant main effect of beverage group $(p>.1)$, or any significant interaction between response type and beverage group $(p>.1)$, instead implies that there was no meaningful difference in the pattern of SCRs observed in each condition. 


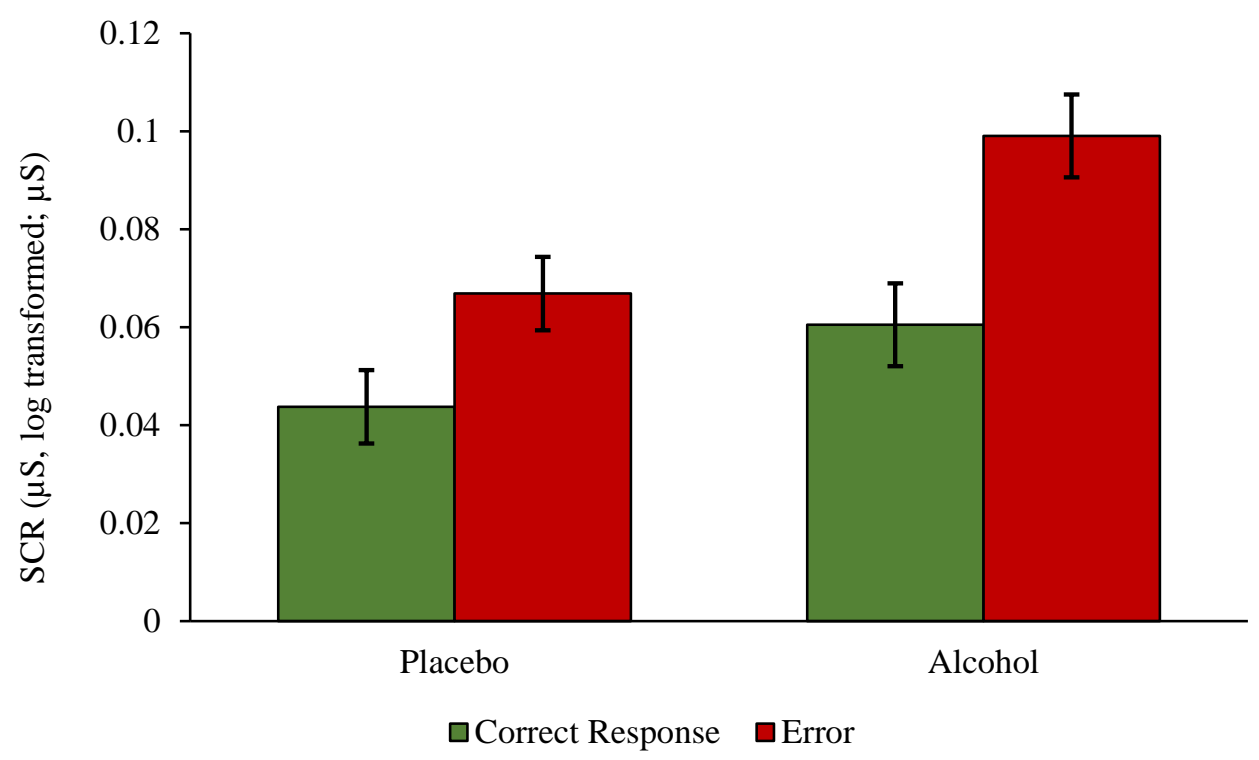

Figure 16. Mean log-transformed skin conductance responses $(\mu S)$ made to correct responses and errors, within each condition. Error bars show the standard error of the mean, normalized and corrected for a within-subjects design (Morey, 2008).

Figure 17 shows the HR response over time following errors and correct responses, within each condition. HR data was entered into 2 (response type) x 6 (time) x 2 (beverage group) mixed ANOVA. As expected, a main effect of response type was found $\left(F(1,68)=57.879, p<.001, \eta_{\mathrm{p}}^{2}=.460\right)$, demonstrating that within both groups, errors elicited greater HR deceleration than correct responses. However, a significant interaction was also found between response type and time $(F(1.815$, $123.399)=18.386, p<.001, \eta_{\mathrm{p}}^{2}=.213$, Greenhouse-Geisser correction applied due to violation of sphericity), indicating that the degree to which errors elicited greater deceleration than correct responses varied across the $3 \mathrm{~s}$ period. However, given that the overall direction of the effect did not change over time, and that the effect of time is not directly relevant to the hypothesis, I decided not to investigate this interaction further. Consistent with the SCR data, there was no evidence of a decreased HR response to errors in the alcohol group. No significant main effect of beverage was 
found within the ANOVA, nor did beverage group significantly interact with any other combination of variables (all $p>.1$ ). Contrary to my prediction that alcohol would reduce the physiological correlates of negative affect elicited by errors, neither SCRs nor HR responses to errors differed between conditions, suggesting that alcohol does not reduce the negative affect generated by errors.

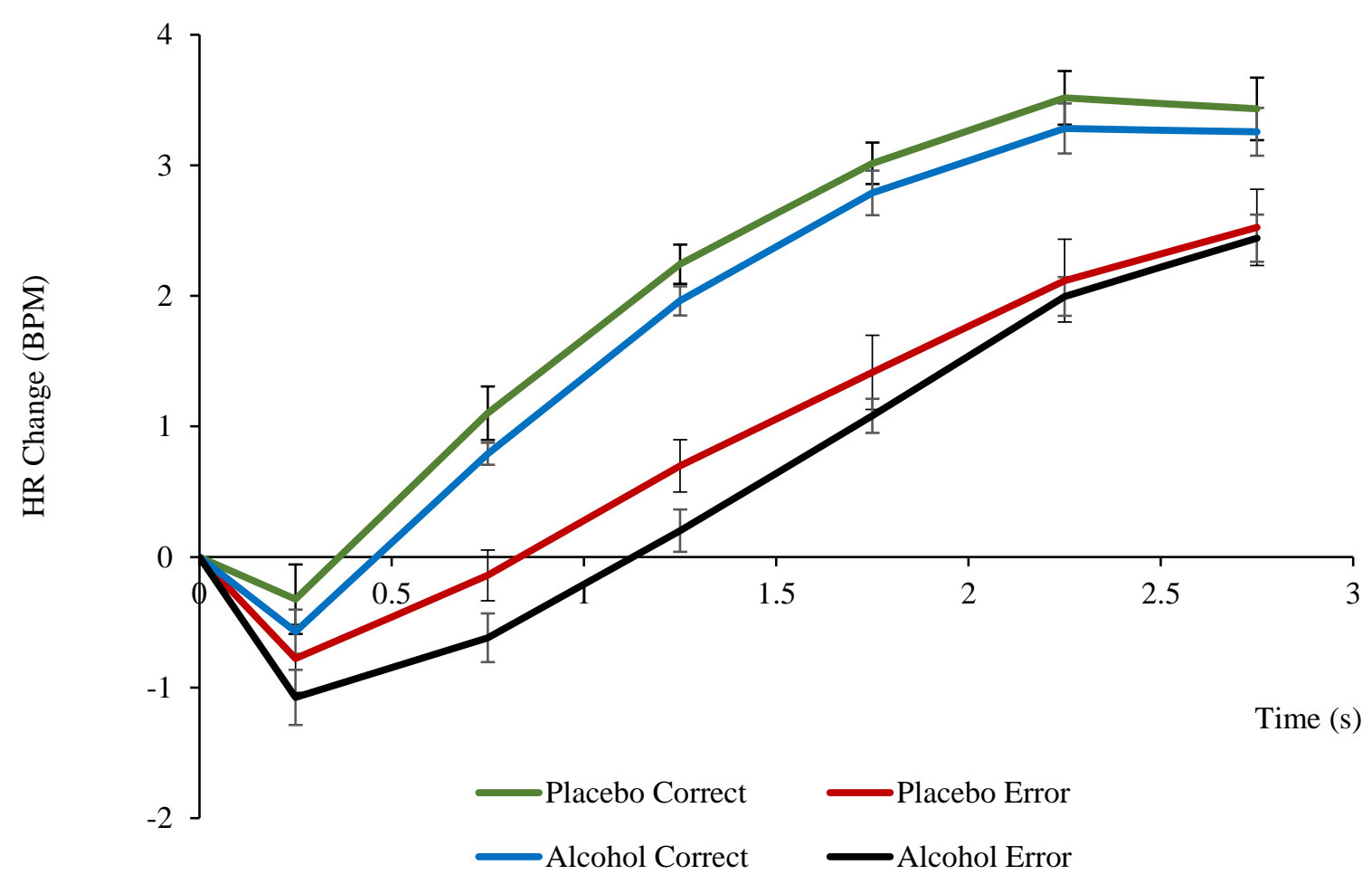

Figure 17. Mean change in HR (BPM) from pre-response baseline over the 3 s following correct responses and errors, within each condition. Error bars show the standard error of the mean, normalized and corrected for a within-subjects design (Morey, 2008).

\section{Electrophysiological Measures}

Figure 18 displays the mean voltage recorded at the FCz electrode within the $650 \mathrm{~ms}$ epoch in which data was analysed, for correct responses and errors, within each condition. To compare the size of the ERN between conditions, peak negative voltage values from within 
the $100 \mathrm{~ms}$ following a response were entered into a 2 (response type) x 2 (beverage group) mixed ANOVA. A significant main effect of response type confirmed the presence of an ERN within both beverage groups $\left(F(1,67)=180.092, p<.001, \eta_{\mathrm{p}}{ }^{2}=.729\right)$. However, there was no interaction between response type and condition $(p>.1)$, indicating that, in contrast to previous studies (Ridderinkhof et al., 2002; Bailey et al., 2014; Bartholow et al., 2012) and my predictions, alcohol did not reduce the size of the ERN.

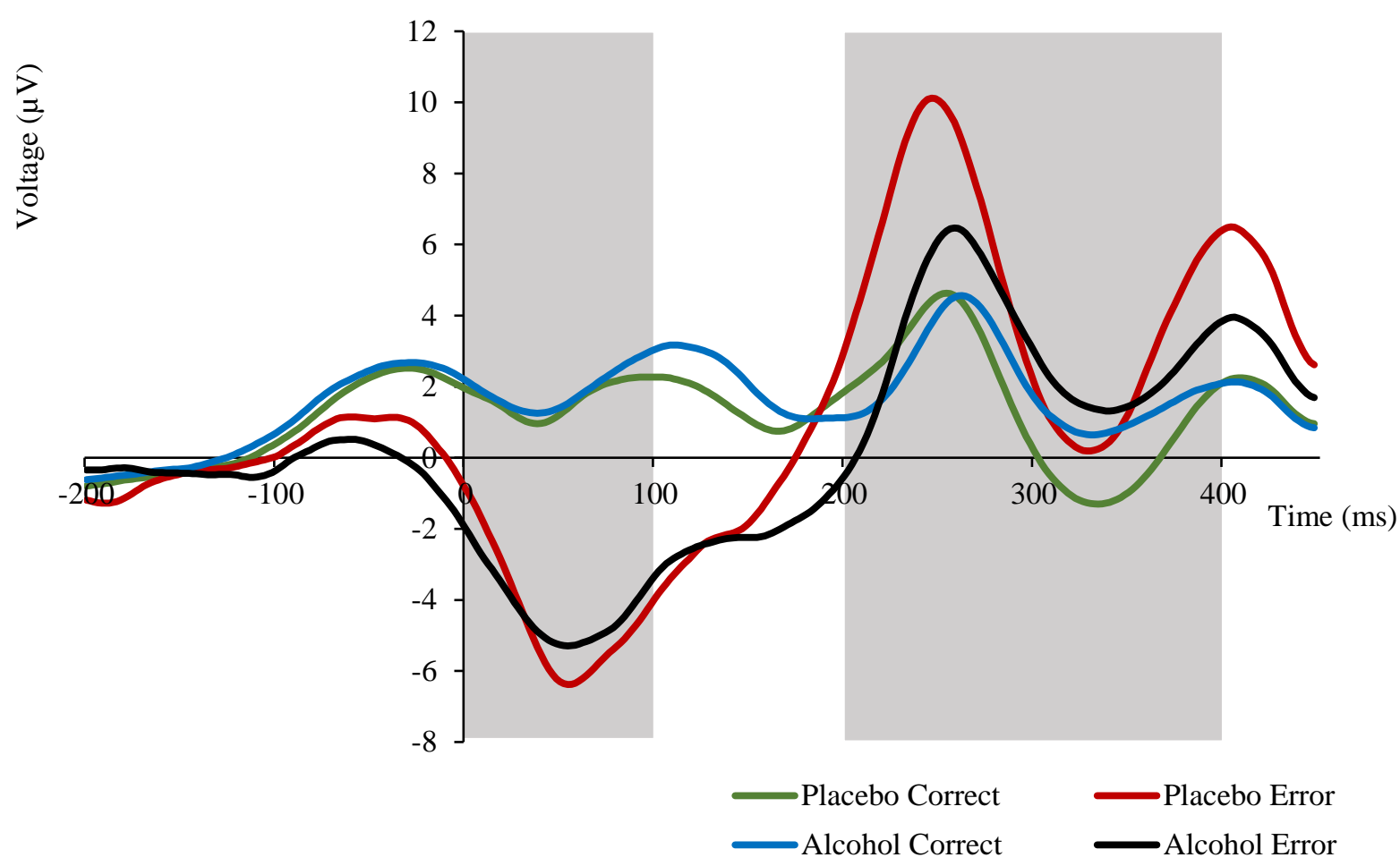

Figure 18. Event related potentials elicited by correct responses and errors, within each condition, at the FCz electrode. The shaded areas represent the time windows in which the ERN $(0-100 \mathrm{~ms})$ and FRN $(200-400 \mathrm{~ms})$ were identified.

However, while beverage group did not influence the magnitude of the ERN, visual examination of activity at FCz suggested substantial differences between conditions in errorrelated brain potentials from $200 \mathrm{~ms}$ post-response onward. To determine whether the beverage groups differed in respect to the size of the FRN, which I defined as the voltage difference between positive and negative peaks occurring between 200 and 400ms post- 
response, results were entered in a 2 (response type) x 2 (beverage group) mixed ANOVA. A significant interaction was found between response type and condition $(F(1,67)=9.350, p=$ $\left..003, \eta_{\mathrm{p}}{ }^{2}=.122\right)$ suggesting that the FRN, indicated by a greater peak-to-trough drop in voltage following errors, was larger in the placebo group. This was confirmed by a follow-up independent samples t-test $(t(67)=3.533, p=.001, d=.878)$. Overall, ERP results imply that alcohol did not reduce early ACC responses to internally detected errors, but that it did attenuate later ACC control signals elicited by negative feedback.

Further evidence for a reduction in ACC responses to error feedback by alcohol was provided by time-frequency analysis of theta band activity. Figures 19 and 20 display fluctuations in participants' midfrontal theta power, in the $2000 \mathrm{~ms}$ before and after correct responses and errors, within each beverage group. To investigate the effect of alcohol on ACC responses to feedback, theta power values measured between 100 and 500ms following a response were analysed in a 2 (response type) x 2 (beverage group) mixed ANOVA. A significant interaction between response type and beverage group was found $(F(1,67)=$ $\left..16 .136, p<.001, \eta_{\mathrm{p}}^{2}=.194\right)$, suggesting that the increase in theta power following errors observed in the placebo group was weakened by alcohol. A follow-up independent samples ttest confirmed that post-error theta power was diminished in the alcohol condition $(t(67)=$ $3.748, p<.001, d=.903)$. Conversely, theta power following correct responses did not differ between beverage groups $(p>.1)$. While alcohol had no effect on the size of the ERN, both the FRN and theta power data suggest that it nevertheless reduced the output of the ACC following errors in some fashion. 

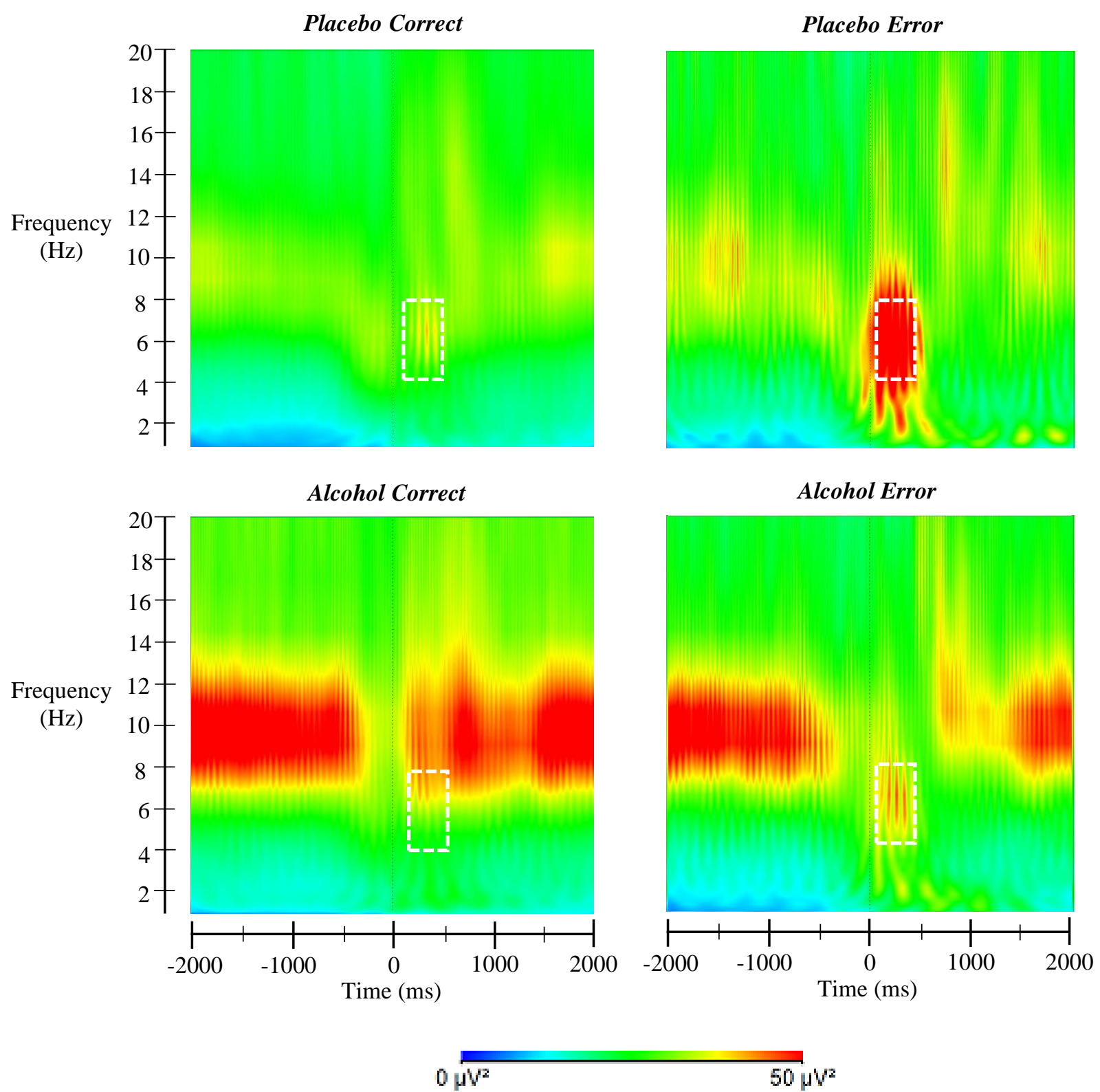

Figure 19. Heatmaps displaying power estimates $\left(\mu V^{2}\right)$ produced by time-frequency analysis of correct responses and errors, within each condition, at the FCz electrode. Maps display the $2000 m$ before and after a response. White-dashed boxes highlight time-frequency bands from which theta power was calculated for the purpose of analysis $(100-400 \mathrm{~ms} ; 4.1-7.8 \mathrm{~Hz})$. 


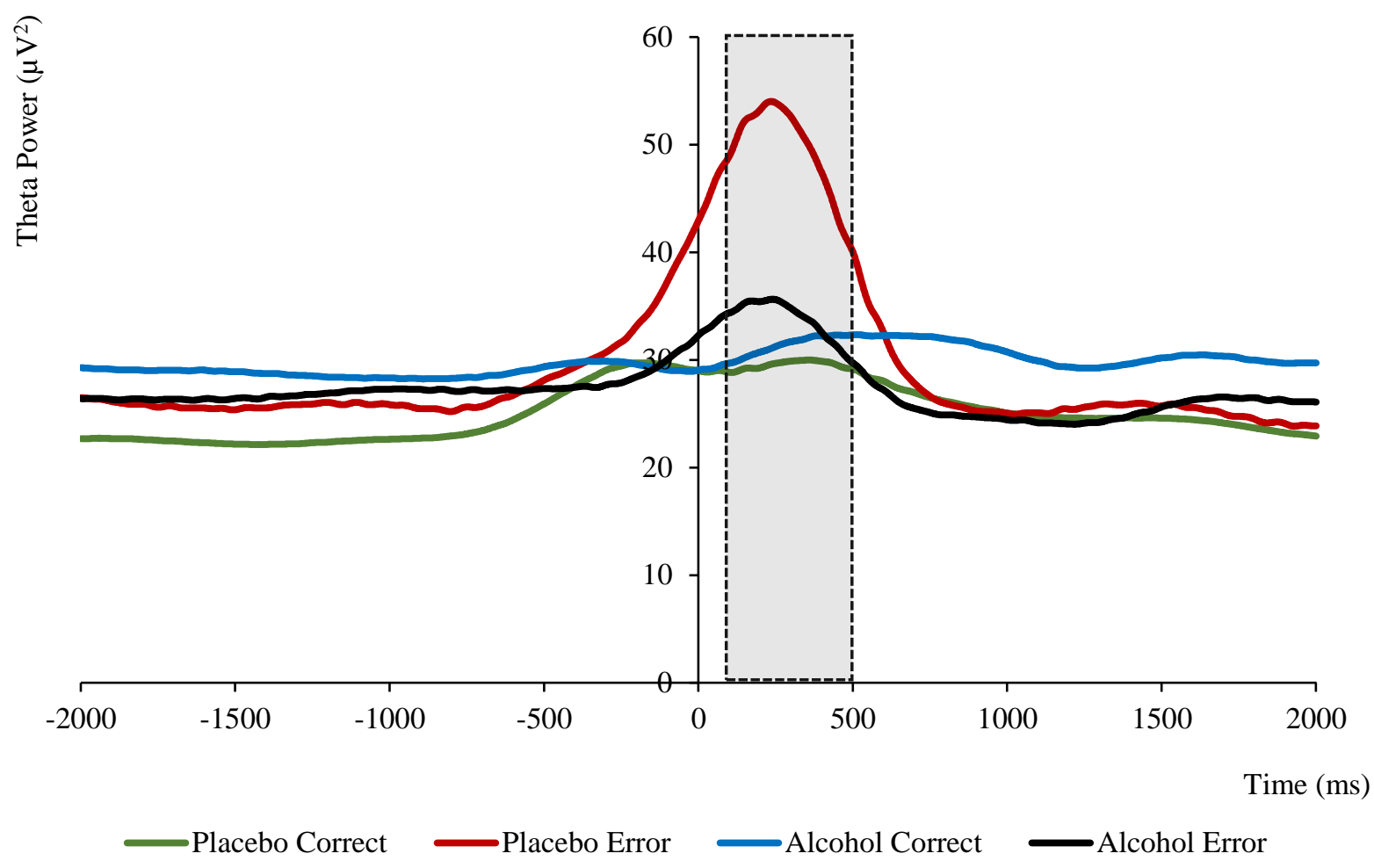

Figure 20. Plot tracking theta power $\left(\mu V^{2}\right)$ over the 2000ms before and after a correct response or an error, within each condition, at the FCz electrode. Shaded area represents the time window (100500ms) during which theta power was analysed.

Figures 21 and 22 display changes in participants' occipital alpha power, in the 2000ms before and after correct responses and errors, within each condition. As the distribution of participants' alpha power was highly skewed, alpha power values were transformed into their base-10 logarithms, before being analysed in a 2 (response type) $\mathrm{x} 4$ (time) $\mathrm{x} 2$ (beverage group) mixed ANOVA. A main effect of beverage group was found $\left(F(1,67)=5.953, p=.017, \eta_{\mathrm{p}}^{2}=.082\right)$, indicating that alcohol increased participants' tonic alpha, suggestive of a sustained deficit in attention. Additionally, a significant main effect of time $\left(F(1.379,92.397)=17.014, p<.001, \eta_{\mathrm{p}}^{2}=.203\right.$, Greenhouse-Geisser correction applied due to violation of sphericity), demonstrated that participants showed phasic changes 
in alpha power across the duration of a trial. Visual examination suggests that alpha power dropped following presentation of flanker stimuli, consistent with attentional engagement with the task, increasing again after feedback was viewed. However, within the ANOVA, time also interacted significantly with response type $(F(2.261,151.456)=7.270, p=.001$, $\eta_{\mathrm{p}}^{2}=.098$, Greenhouse-Geisser correction applied due to violation of sphericity), indicating that the pattern of this phasic change differed based on whether participants made a correct or an erroneous response on the trial. As I was primarily interested in whether participants were suppressing alpha waves after an error, I followed up this interaction with a paired samples ttest comparing alpha power in the $500-700 \mathrm{~ms}$ epoch after errors and correct responses (averaging over beverage group). A significant difference was found $(t(68)=4.751, p<.001$, $d_{z}=.571$ ), suggesting that alpha power was suppressed following task errors, consistent with post-error cognitive adaptation. Furthermore, results from additional t-tests demonstrated that alpha power did not differ based on response type at any other time points (all $p>.1$ ). Beverage group did not interact significantly with any combination of other variables (all $p$ > $.1)$, indicating that this pattern of post-error alpha suppression was not altered by alcohol.

Time-frequency analysis of occipital alpha power largely corroborated the inferences drawn from my behavioural data: alcohol reduced participants' overall use of cognitive control (as indicated by their higher levels of alpha across trials), while not affecting the degree to which cognitive control was upregulated immediately following an error (as indicated by their similar phasic responses to errors). 

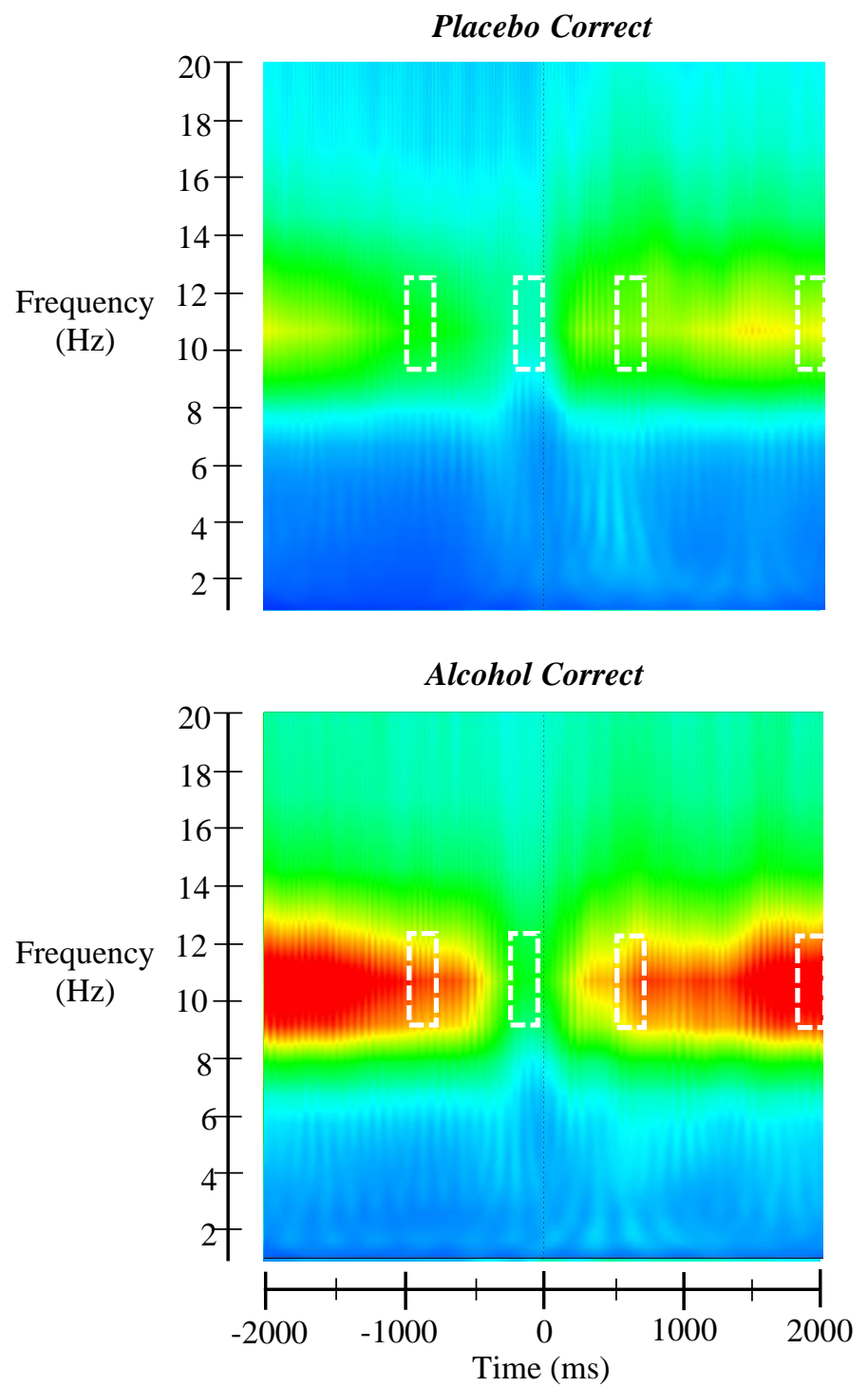

Placebo Error

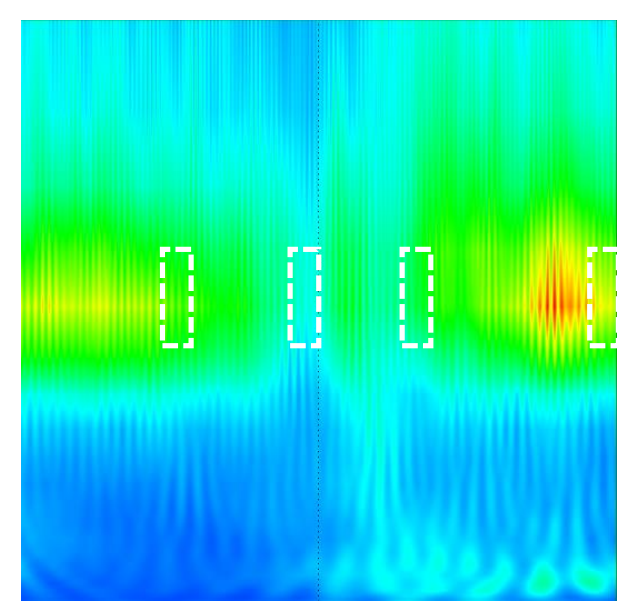

Alcohol Error

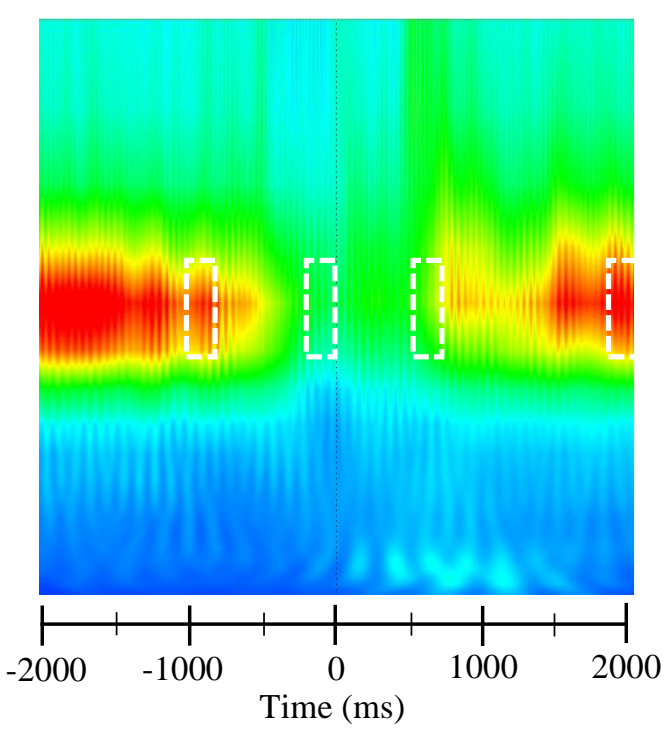

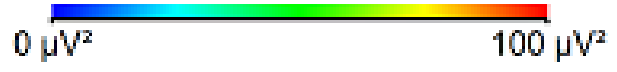

Figure 21. Heatmaps displaying power estimates $\left(\mu V^{2}\right)$ produced by time-frequency analysis of correct responses and errors, within each condition, at the Oz electrode. Maps display the 2000ms before and after a response. White-dashed boxes highlight time-frequency bands from which alpha power was calculated for the purpose of analysis (-1000--800ms, -200-0ms, 500-700ms, 1800-2000ms; 9.1 $-12.5 \mathrm{~Hz})$. 


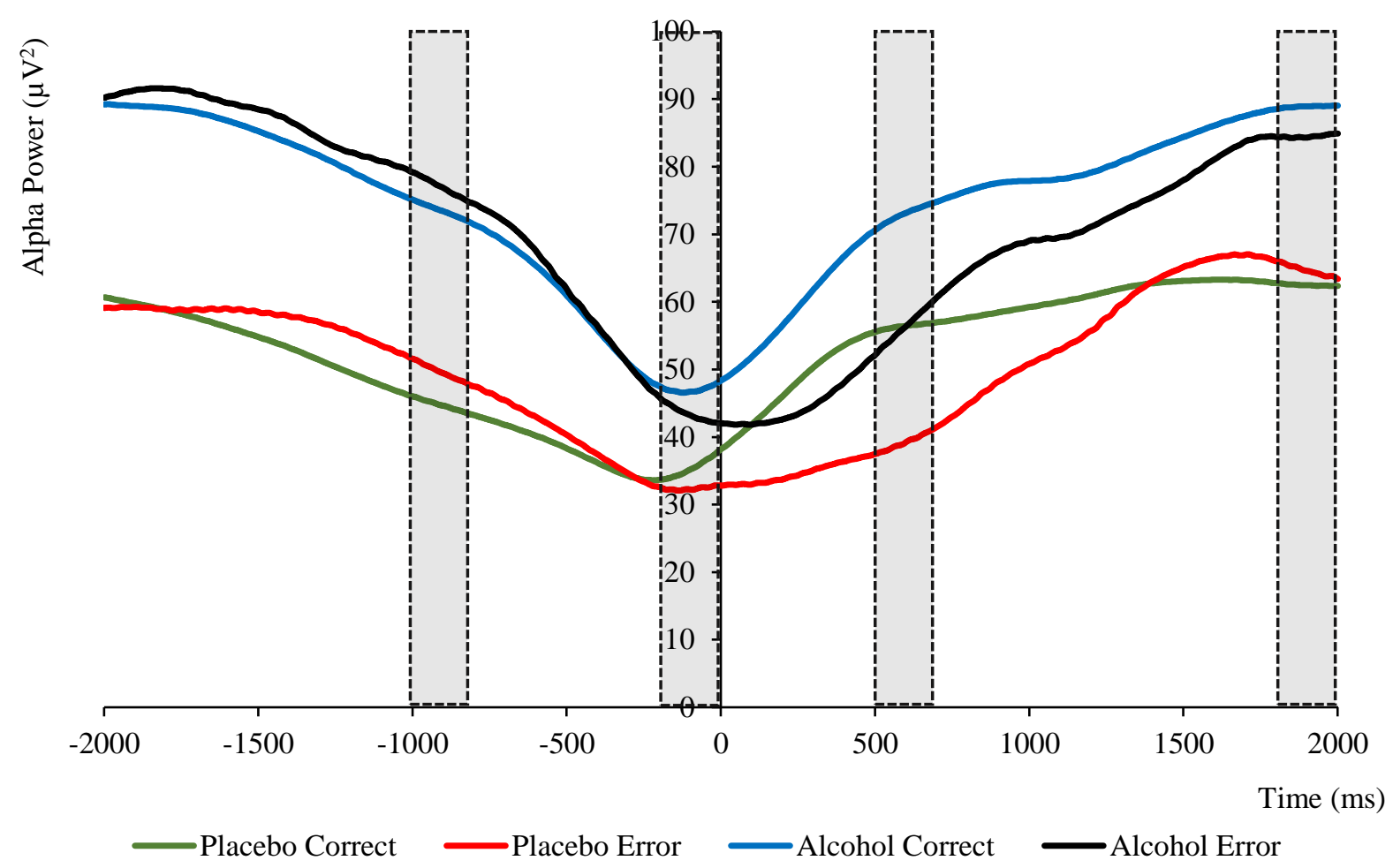

Figure 22. Plot tracking alpha power $\left(\mu V^{2}\right)$ over the 2000ms before and after a correct response or an error, within each condition, at the Oz electrode. Shaded area represent the time windows (-1000-800ms, -200-0ms, $500-700 m s, 1800-2000 m s)$ during which alpha power was analysed.

\section{Correlational Data}

Table 4 displays bivariate correlations between physiological responses to errors, changes in state affect as indexed by the PANAS, and behavioural measures of post-error adaptation. In contrast to my first experiment, no relationship was found between SCR or HR change following errors, and PES or PERI. Furthermore, there was no correlation between change in negative affect and post-error adaptation, as might be predicted based on Bartholow and colleague's (2012) findings. This suggests that in this experiment, negative affect was not driving participants' ability to upregulate cognitive control after an error. I did find a marginally significant correlation between participants' changes in negative affect 
(between arriving in the lab, and ten minutes after beverage consumption) and post-error theta power (i.e. a smaller decrease in negative affect predicted greater theta power), consistent with previous studies showing that ACC response to errors can be modulated by negative emotion (e.g. Wiswede et al., 2009).

However, I was concerned that any actual relationship between negative affect and post-error adaptation within the placebo group might have been obscured by the lack of such a relationship in the alcohol group, or vice versa. Therefore, I also computed bivariate correlations for each condition separately. Results can be seen in table 5 and table 6 . For both beverage groups, the overall pattern of correlations was similar to what was observed when conditions were combined; no relationship was found between physiological responses to errors or self-reported negative affect, and either PES or PERI. Within the placebo group, a correlation was once again found between change in negative affect and post-error theta power. However, this relationship did not hold within the alcohol group.

I had originally planned to carry out a mediation analysis, via structural equation modelling (see Bartholow et al., 2012), to test whether changes in negative affect were driving differences in post-error adaptation between beverage groups. However, as there were no correlations between, or group differences in, measures of affect and measures of posterror adaptation, I had no reason to perform such an analysis. 
Table 4. Correlations between psychophysiological responses to errors (HR, SCR, ERN, theta power), changes in PANAS ratings, and measures of post-error adaptation (PES, PERI).

\begin{tabular}{|c|c|c|c|c|c|c|c|}
\hline & NA change & PA change & SCR error & ERN FCz & $\begin{array}{l}\text { Theta } 100 \\
-500 \mathrm{~ms} \\
\text { error }\end{array}$ & PES & PERI \\
\hline $\begin{array}{l}\Delta \mathrm{HR} 0-.5 \mathrm{~s} \\
\quad \text { error }\end{array}$ & .152 & .052 & -.195 & .086 & -.044 & .165 & .057 \\
\hline $\begin{array}{l}\Delta \mathrm{HR} .5-.1 \mathrm{~s} \\
\quad \text { error }\end{array}$ & .087 & .025 & .041 & .032 & .048 & .128 & .107 \\
\hline $\begin{array}{l}\Delta \mathrm{HR} 1-1.5 \mathrm{~s} \\
\quad \text { error }\end{array}$ & .035 & -.060 & .015 & .060 & .107 & .093 & .109 \\
\hline $\begin{array}{c}\Delta \mathrm{HR} 1.5-2 \mathrm{~s} \\
\text { error }\end{array}$ & .051 & -.155 & .086 & .085 & .134 & .043 & .076 \\
\hline $\begin{array}{c}\Delta \mathrm{HR} 2-2.5 \mathrm{~s} \\
\text { error }\end{array}$ & .090 & -.179 & .125 & .104 & .106 & .021 & .102 \\
\hline $\begin{array}{c}\Delta \mathrm{HR} 2.5-3 \mathrm{~s} \\
\text { error }\end{array}$ & .112 & -.155 & .185 & .072 & .075 & .000 & .135 \\
\hline NA change & & -.041 & .070 & -.043 & $.216 \#$ & .088 & -.019 \\
\hline PA change & & & .038 & -.131 & -.187 & .072 & -.061 \\
\hline SCR error & & & & -.181 & .030 & -.129 & -.138 \\
\hline ERN FCz & & & & & $-.342 * *$ & -.200 & -.022 \\
\hline $\begin{array}{l}\text { Theta } 100- \\
500 \mathrm{~ms} \text { error }\end{array}$ & & & & & & .024 & -.072 \\
\hline PES & & & & & & & -.002 \\
\hline
\end{tabular}


Table 5. Correlations between psychophysiological responses to errors (HR, SCR, ERN, theta power), changes in PANAS ratings, and measures of post-error adaptation (PES, PERI) for participants within the placebo group only.

\begin{tabular}{|c|c|c|c|c|c|c|c|}
\hline & NA change & PA change & SCR error & ERN FCz & $\begin{array}{c}\text { Theta } 100 \\
-500 \mathrm{~ms} \\
\text { error }\end{array}$ & PES & PERI \\
\hline $\begin{array}{l}\Delta \mathrm{HR} 0-.5 \mathrm{~s} \\
\quad \text { error }\end{array}$ & $.316 \#$ & .098 & -.109 & -.009 & -.085 & .287 & .019 \\
\hline $\begin{array}{l}\Delta \mathrm{HR} .5-.1 \mathrm{~s} \\
\quad \text { error }\end{array}$ & .222 & .038 & .004 & -.020 & -.027 & .231 & .176 \\
\hline $\begin{array}{l}\Delta \mathrm{HR} 1-1.5 \mathrm{~s} \\
\quad \text { error }\end{array}$ & .165 & -.100 & .048 & .035 & .062 & .175 & .188 \\
\hline $\begin{array}{c}\Delta \mathrm{HR} 1.5-2 \mathrm{~s} \\
\text { error }\end{array}$ & .151 & -.205 & .064 & .037 & .108 & .115 & .151 \\
\hline $\begin{array}{c}\Delta \mathrm{HR} 2-2.5 \mathrm{~s} \\
\text { error }\end{array}$ & .196 & -.234 & .105 & .042 & .106 & .085 & .141 \\
\hline $\begin{array}{c}\Delta \mathrm{HR} 2.5-3 \mathrm{~s} \\
\text { error }\end{array}$ & .209 & -.203 & .125 & .055 & .068 & .064 & .152 \\
\hline NA change & & -.073 & -.066 & -.046 & $.438 *$ & .078 & -.054 \\
\hline PA change & & & -.028 & -.261 & $-.324 \#$ & .204 & .013 \\
\hline SCR error & & & & $-.316 \#$ & .021 & -.049 & .064 \\
\hline ERN FCz & & & & & $-.361 *$ & -.230 & -.086 \\
\hline $\begin{array}{l}\text { Theta } 100- \\
500 \mathrm{~ms} \text { error }\end{array}$ & & & & & & .025 & -.108 \\
\hline PES & & & & & & & -.190 \\
\hline
\end{tabular}


Table 6. Correlations between psychophysiological responses to errors (HR, SCR, ERN, theta power), changes in PANAS ratings, and measures of post-error adaptation (PES, PERI) for participants within the alcohol group only.

\begin{tabular}{|c|c|c|c|c|c|c|c|}
\hline & NA change & PA change & SCR error & ERN FCz & $\begin{array}{l}\text { Theta } 100 \\
-500 \mathrm{~ms} \\
\text { error }\end{array}$ & PES & PERI \\
\hline $\begin{array}{l}\Delta \mathrm{HR} 0-.5 \mathrm{~s} \\
\quad \text { error }\end{array}$ & -.173 & .111 & $-.292 \#$ & $.360^{*}$ & -.266 & -.069 & .082 \\
\hline $\begin{array}{l}\Delta \mathrm{HR} .5-.1 \mathrm{~s} \\
\quad \text { error }\end{array}$ & -.244 & .144 & -.067 & .247 & -.089 & -.110 & -.128 \\
\hline $\begin{array}{l}\Delta \mathrm{HR} 1-1.5 \mathrm{~s} \\
\quad \text { error }\end{array}$ & $-.303 \#$ & .121 & .017 & .197 & .062 & -.102 & -.138 \\
\hline $\begin{array}{c}\Delta \mathrm{HR} 1.5-2 \mathrm{~s} \\
\quad \text { error }\end{array}$ & -.236 & -.024 & .182 & .253 & .158 & -.126 & -.144 \\
\hline $\begin{array}{c}\Delta \mathrm{HR} 2-2.5 \mathrm{~s} \\
\quad \text { error }\end{array}$ & -.225 & -.086 & .201 & .264 & .119 & -.113 & .004 \\
\hline $\begin{array}{c}\Delta \mathrm{HR} 2.5-3 \mathrm{~s} \\
\quad \text { error }\end{array}$ & -.131 & -.082 & $.301 \#$ & .115 & .106 & -.114 & .100 \\
\hline NA change & & -.123 & .208 & -.121 & -.077 & .159 & .115 \\
\hline PA change & & & .028 & -.085 & $.367^{*}$ & -.018 & -.096 \\
\hline SCR error & & & & -.115 & .238 & -.180 & -.328 \\
\hline ERN FCz & & & & & -.216 & -.129 & -.133 \\
\hline $\begin{array}{l}\text { Theta } 100- \\
500 \mathrm{~ms} \text { error }\end{array}$ & & & & & & -.135 & -.212 \\
\hline PES & & & & & & & .236 \\
\hline
\end{tabular}




\section{Discussion}

Experiment 2 tested the hypothesis that the reduction of post-error adaptation by alcohol is caused by a reduced emotional impact of errors. Based on this hypothesis, I made four predictions: firstly, that alcohol would decrease the magnitude of SCR and HR deceleration following errors; secondly, that alcohol would reduce measures of post-error adaptation (PES, PERI and alpha suppression), thirdly, that alcohol would decrease the size of the ERN; and finally, that differences in physiological responses to errors would mediate observed differences in post-error adaptation between alcohol and placebo participants. None of these predictions were supported by the data, providing strong evidence against my overall hypothesis.

In terms of accuracy and error rate, alcohol group participants were not any worse at performing the flanker task than placebo group participants. However, alcohol did increase the interference with response time produced by incongruent flanker strings, suggesting that it may have disrupted participants' ability to sustain the use of cognitive control over the course of the task. This interpretation is supported by my time-frequency analysis of participants alpha power, which found a lower tonic level of alpha within the alcohol condition. This indicates that alcohol group participants were less attentive and engaged with the task overall, consistent with a general impairment of cognitive control.

However, in contrast to Ridderinkhof (2002), Bailey (2014) and Bartholow (2012), I found no evidence to suggest that alcohol specifically disrupted participants' ability to upregulate control after making an error. Alcohol group participants did not display decreased PERI, PES or post-error alpha suppression. Moreover, a case could be made that neither the alcohol nor placebo participants actually increased control after making an error. Firstly, there was no evidence of PERI in either group. Secondly, while both groups showed PES, without other sources of evidence in support, it is impossible to determine whether this 
reflects an adaptive shift in control strategy, or alternatively, a general disruption of performance due competition for attentional resources from error feedback (see Castellar et al., 2010; Notebaert et al., 2009). Likewise, occipital alpha is fundamentally a measure of attention. While alpha suppression may have occured following errors because control was being used to facilitate increased attentional engagement with the task, it could also have happened because of attentional orienting towards error feedback, was rarer and presumably more emotionally salient than correct feedback. This interpretation is consistent with the relatively short time frame during which post-error alpha suppression was displayed, lasting for only around $1500 \mathrm{~ms}$, well before the beginning of the next trial (see figure 21 and figure 22).

I also found no evidence that alcohol decreased negative affect, in terms of either overall mood, or physiological response to errors. Unlike Bartholow and colleagues (2012), alcohol consumption did not decrease my participants' self-reported negative affect. Furthermore, my physiological indices of participants' emotional response to errors, SCR and HR deceleration, were not only not reduced by alcohol, but actually trended towards being larger within the alcohol group. This outcome seems to refute the claim that alcohol reduces the negative affect generated by errors, and provides evidence against my overall hypothesis.

Additionally, the amplitude of participants' ERN was not diminished by alcohol, also in contrast to previous research (i.e. Bailey et al., 2014; Bartholow et al., 2002; Ridderinkhof et al., 2002). I did however, find that alcohol decreased the size of the FRN component, and theta power between 100 and 500ms after an error. This is somewhat unusual as all three measures should, in principle, index the output of the ACC (Cavanagh et al., 2012), perhaps suggesting that while alcohol did not reduce participants' rapid initial ACC response, based on endogenous evaluation of incorrect motor commands, it did reduce sustained activation of 
the ACC in response to negative feedback. I will discuss some potential causes of this disparity in my general discussion.

Finally, in contrast to both Bartholow and colleagues (2012), and the results of my first experiment, neither state negative affect nor affective responses to errors predicted participants' levels of post-error compensatory behaviour, within either the alcohol or placebo conditions. This finding not only does not support the hypothesis that a reduction in negative affect drives alcohol's effects on post-error adaptation, it furthermore suggests that neither group of participants utilized the emotional information provided by errors to guide corrective cognitive adjustment.

On the whole, my results of my second experiment were surprising, and run contrary to what would be expected if my original hypothesis was correct. In my general discussion I will explore in depth some of reasons why I may have obtained the results that I did, and what this might tell us more generally about the influence of alcohol on cognition.

\section{General Discussion}

The behavioural dysregulation created by alcohol may stem from an impairment of evaluative cognitive control, that is, the ability to regulate the use of control strategies based on the needs of the current environment. This view is supported by studies showing that alcohol reduces both ACC responses and behavioural compensation which normally follow errors in conflict tasks (Bailey et al., 2014; Marinkovic et al., 2013; Ridderinkhof et al., 2002). However, the underlying mechanism by which alcohol might disrupt an ACC based control response to errors, and the significance of this disruption in real world settings, remains unclear. Bartholow and colleagues (2012) argue that the capacity of alcohol to impair post-error adaptation is driven by a reduction of the negative affect that errors normally generate. In this thesis, I carried out two experiments to test this hypothesis. In my first experiment I examined participants' physiological and behavioural changes following errors 
in a flanker task, in order to validate an experimental paradigm that could then be used to test intoxicated participants. Results replicated previous research showing that errors elicit changes in SCR and HR consistent with negative emotion (Hajcak et al., 2003b, 2004). Furthermore, I found that the size of these ANS responses was correlated with participants' ability to reduce flanker interference following an error, supporting theoretical models linking the adjustment of cognitive control with a drive to reduce negative affect (i.e. Inzlicht et al., 2015, Saunders et al., 2017).

In my second experiment, I directly tested Bartholow and colleagues' model by comparing physiological and behavioural responses to flanker errors in participants receiving either an alcoholic or placebo beverage. The results of my second experiment were surprising in several respects, running counter to what I had predicted on the basis of both my first experiment and the broader literature. Firstly, while alcohol did cause an overall increase in flanker interference, alcohol and placebo participants showed no discernible difference in the degree to which they increased the use of cognitive control after making an error. Secondly, in marked contrast to previous research (i.e. Bailey et al., 2014; Bartholow et al., 2012; Ridderinkhof et la., 2002), alcohol did not reduce the amplitude of the ERN, although it did attenuate two exploratory measures of ACC output, the FRN and midfrontal theta. Thirdly, alcohol did not reduce participants' state negative affect, nor did it decrease SCR or HR deceleration following errors. Finally, the correlations between ANS response to errors and PERI observed in my first experiment were not replicated, even within the placebo group. In the remainder of this thesis, I will explore some of the reasons why my results may have differed from the results of previous studies, assess the wider implications of my findings for understanding the effects of alcohol on behaviour and cognitive control, and discuss possible directions for future research into this subject. 


\section{Why No Effect of Alcohol on Post-Error Adaptation?}

Previous research has found that alcohol reduces behavioural indicators of increased cognitive control following errors. Ridderinkhof (2002) and Bailey (2014) both found that alcohol decreased PERI within a flanker task. Additionally, Bartholow and colleagues (2012) found that alcohol prevented the improvement in accuracy which normally followed an error on a weapon identification task, while Marinkovic and colleagues (2013) found that intoxicated subjects were less likely to correct an erroneous eye movement in an antisaccade task. In contrast, alcohol group participants in my second experiment showed no sign of reduced post-error behavioural adaptation, in terms of either PES or PERI. Additionally, I found that the degree to which participants' occipital alpha power dropped following an error was also unchanged between conditions, further suggesting that alcohol was not worsening my participants' ability to upregulate cognitive control in response to a mistake.

One possible cause of these apparently conflicting findings was the inclusion of feedback following each response, in the version of the flanker task used in my experiment. Ridderinkhof and colleagues (2002) argue that alcohol impairs the monitoring functions of the ACC, preventing it from recognising when errors are made and increasing control in response. While this model is seemingly at odds with studies indicating that intoxicated individuals can accurately evaluate their performance (see Bailey et al., 2014; Bartholow et al., 2012), it would offer a possible explanation for the absence of alcohol effects on posterror adaptation, within my experiment. Specifically, giving explicit, immediate and clearly visible feedback likely meant that all of my participants, intoxicated or sober, were well aware when they had made an error. If alcohol primarily affects whether an error is detected, rather than directly modulating the strength of the subsequent control signal, we would then expect feedback which makes all errors detectable to guard against any impairment of post- 
error upregulation of control by alcohol. This may therefore explain why alcohol did not reduce post-error adaptation in my experiment.

However, as previously discussed, there is reason to be sceptical as whether any participants in my study upregulated cognitive control immediately after making an error: neither beverage group demonstrated PERI, and the observation of both PES and post-error alpha suppression could also be explained by attentional orienting towards feedback, rather than cognitive adaptation. If this is true, it would suggest that the reason alcohol did not reduce behavioural measures of post-error adaptation in my experiment is because there were no underlying short-term shifts in cognitive control to be reduced. What could have prevented post-error control adjustments within my task? Aside from feedback, another way in which my flanker task was atypical was the extended (up to $4 \mathrm{~s}$ ) interval between a participants' response and the next trial. I chose to include such a long response-stimulus interval (RSI) so that there would be time for trial-to-trial changes in heart rate and electrodermal activity to be measured. However, this could also have inadvertently influenced the difficulty of effectively adjusting control in the task, or the type of strategies by which this was accomplished. PES is known to decrease as RSI increases (Danielmeier \& Ullsperger, 2011), suggesting that the upregulation of control following an error may be a transient phenomenon and not readily sustained over long periods of time.

Alternatively, rather than preventing the post-error upregulation of control, the extended RSI in my study may have instead altered the type of control adjustments prompted by errors. Adjustments in cognitive control can be both reactive, in which control is immediately increased when task performance is threatened, or proactive, in which long-term task strategies are altered, thereby precluding the need for later reactive adjustments (Braver, 2012). Ridderinkhof (2002) found that within a conflict task, PERI was only observed when congruent trials outnumbered incongruent trials, disappearing when incongruent trials 
predominated. He argued that the high likelihood of further conflict encouraged participants to implement proactive adjustments in control when struggling with the task, eliminating the need for trial-to-trial increases in control. A similar process may have been at work in my study; the long RSI might have encouraged participants to make long-term strategic adjustments when their performance proved inadequate, rather than attempt to sustain shortterm control over interference until the next trial. Furthermore, an impairment of evaluative control by alcohol could have disrupted this sustained modulation of cognitive control. This would fit with the overall increased flanker interference and tonic alpha power observed in my alcohol group participants. Note that, in contrast to my findings, neither Ridderinkhof (2002) nor Bailey (2014) observed any overall difference in flanker interference between their alcohol and control participants, despite both finding disparities in post-error behavioural adjustment.

Nonetheless, given the absence of any observable differences post-error adaptation between alcohol and placebo participants, or indeed any strong evidence for post-error upregulation of control in general, it must be concluded that the task I designed was ultimately ill-suited for determining the mechanisms by which alcohol might affect evaluative control. Although the changes I made to the task were seemingly justified given the specific question I was attempting to investigate, in hindsight, they may have inadvertently undermined this investigation. The possible elimination of post-error adaptation by an extended RSI is particularly concerning; measuring SCR or HR deceleration, along with most other possible indices of negative affect, requires a long RSI, potentially preventing the simultaneous monitoring of post-error behavioural adjustments.

However, we should also consider the more general limitations associated with using post-error behavioural adaptations as a research tool. Along with the aforementioned difficulties in interpreting PES, both PES and PERI are relatively unreliable measures, and 
are seemingly highly sensitive to the precise parameters of a task (Compton, Heaton \& Gaines, 2018; Notebaert et al., 2009). Although research on alcohol and evaluative control has hitherto relied primarily on these measures of upregulated control, future studies may be better served by developing more direct and reliable indices. One option might be to employ tasks in which errors can be corrected, provided control resources are quickly engaged. The anti-saccade task may be a possible candidate, given Marinkovic and colleagues' (2013) observation that alcohol can reduce the degree to which participants compensate for erroneous eye movements in the task.

\section{Why No Effect of Alcohol on the ERN?}

The potential for alcohol to reduce the amplitude of the ERN, and the ACC's response to errors more generally, is well supported by existing research (e.g. Anderson et al., 2011; Bailey et al., 2014; Easdon et al., 2005; Ridderinkhof et al., 2002). However, while alcohol did reduce the size of the FRN and post-error theta power in my second experiment, the amplitude of the ERN did not differ between beverage groups. As with post-error adaptation, this lack of difference might be explained by the inclusion of feedback in my experiment. If Ridderinkhof and colleagues (2002) are correct in suggesting that alcohol attenuates the ERN because it creates deficits in error detection, then feedback, which in principle should make errors easy for all participants to notice, might then preclude any such attenuation.

However, I would argue that this explanation is unconvincing for two reasons. Firstly, I found a very clear effect of alcohol on the FRN and post-error theta power. This suggests that alcohol did alter ACC responses to negative feedback, which is inconsistent with a model in which alcohol only compromises error detection. Although it should be kept in mind that my analyses of the FRN and midfrontal theta were both exploratory rather than confirmatory, the extremely low p-values (both < .001) observed for both effects make a type I error relatively unlikely. Secondly, the short latency of the ERN, along with the fact that it can be observed 
even when sensory input is unavailable (Allain et al., 2004), suggests that it reflects the monitoring of one's own incorrect motor outputs, rather than a response to external stimuli. Therefore, if alcohol reduces the size of ERN because it disrupts this monitoring system, this effect should be unchanged by feedback. Supporting this view, the ERN peaked around 60ms post-response in my experiment, long before any substantial visual processing of feedback was likely to have taken place (Luck, Woodman \& Vogel, 2000).

A more promising explanation for the lack of difference in ERN amplitude between conditions is offered by a study by Olvet and Hajcak (2009). Olvet and Hajcak investigated the effect of performance feedback on the modulation of the ERN by anxiety. They found that higher levels of anxiety predicted an increased ERN amplitude when feedback was absent, consistent with previous research (e.g. Hajack et al., 2003a). However, when they gave feedback to participants this effect disappeared, and greater anxiety instead predicted a larger FRN. This could indicate that in situations where feedback is available, the ACC might wait until this slower, but more reliable source of information on task performance can be processed, before reaching a final judgement on if, and to what extent, control settings should be adjusted. Therefore, in tasks in which feedback is given, slower electrophysiological measures, such as the FRN or midfrontal theta, might more valid indices of overall ACC output. This interpretation suggests that while alcohol didn't reduce the amplitude of the ERN in my study, it nevertheless weakened the overall strength of the control signal produced in the ACC following errors, in a manner consistent with previous research.

\section{Why No Effect of Alcohol on Negative Affect?}

Bartholow and colleagues (2012) claim that alcohol blunts negative affect, including in response to errors. Appropriately, they found that their participants' self-reported state negative affect dropped after they were given alcohol. However, my second experiment not only failed to replicate their finding that alcohol reduced state negative affect, it also clearly 
demonstrated that alcohol does not weaken emotional responses to errors, at least as indexed by physiological changes. This seemingly contradicts Bartholow and colleagues' proposal that alcohol reduces the negative affect generated by errors, and casts further doubt on the idea that alcohol directly lessens negative emotion at all.

However, an alternative explanation for the absence of alcohol-induced reductions in negative affect observed in my second experiment, is that I did not administer a large enough dose of alcohol to reliably produce measurable effects on negative emotion. Bartholow and colleagues (2012) suggest that while relatively low doses of alcohol (i.e. < 0.07\% BAC) have only an indirect effect on emotion, modulating affect only to the degree to which it alters attentional inputs, larger doses do directly reduce negative affect (see also Donohue et al., 2007). Although the veracity of this claim is difficult to evaluate given the sprawling and often inconsistent nature of the literature on alcohol and emotion, this could potentially explain the divergent patterns of results we observed: Bartholow and colleagues (2012) gave their participants sufficient alcohol for their BAC to reach $0.1 \%$, whereas I intended to produce a BAC of only $0.08 \%$ in my alcohol group participants and may have even fallen short of that target. It is therefore possible that if I had administered a larger dose, I would have observed a reduction in state negative affect, and potentially even a decreased physiological response to errors, within my alcohol condition. However, this explanation would still likely rule out the attenuation of negative affect as a possible mechanism for the disruption of post-error adjustments in control by alcohol, as both Ridderinkhof and colleagues (2002), and Marinkovic and colleagues (2013) have previously observed impairments of post-error adaptation at relatively low BACs $(0.04 \%$ and $0.055 \%$ respectively).

However, before we conclude that the impairment of post-error adaption by alcohol cannot be mediated a reduction in negative affect, it is important to consider the limitations of 
using SCR and HR to make inferences about subjects' emotional states. Firstly, it is well known that electrodermal and HR responses to emotional stimuli are driven primarily by changes in arousal, not valence (Bradley, 2009). Therefore, while we can be reasonably sure that alcohol did not reduce the arousal generated by errors in my task, it could nevertheless have reduced the degree to which errors were judged as being unpleasant. Secondly, emotion is not a unitary construct. While changes within the ANS are a vital component of emotion, subjective experience and behaviour are equally important, and can, in some cases, dissociate from physiological responses (Mauss et al., 2005). It has furthermore been argued that the emotions we experience are determined principally through the cognitive attributions we make as to the causes of our current physiological state (Gendron \& Barrett, 2009; Schachter \& Singer, 1962). Within this perspective, alcohol could plausibly leave ANS responses to errors and other aversive stimuli unchanged, while still dramatically altering consequent experiences and behaviours. Furthermore, while theories of ACC function typically focus on emotion as a modulator of control processes, there is also considerable evidence to suggest that the ACC plays an important role in generating emotional experiences, particularly those involving negative emotions (Shackman, Salomons, Slagter, Fox, Winter \& Davidson, 2011). If alcohol disrupts the ACC's response to errors, this could then modify the degree to which an error is perceived as distressing, even as inputs to the ACC specifying the motivational significance of that error remain the same. Further research is therefore needed before we can be certain that alcohol does not reduce the negative affect elicited by errors.

\section{Why No Correlations Between ANS and Behavioural Responses?}

In my first experiment, I observed significant correlations between ANS measures indexing emotional response to errors (i.e. HR and SCR), and PERI, supporting the more general theory that negative affect facilitates cognitive control. However, this relationship failed to replicate in my second experiment, suggesting that participants were no longer using 
the information provided by negative emotion to guide post-error adaptation. It is particularly surprising, that the placebo group did not demonstrate a relationship between ANS responses to errors and PERI, given that they were performing the same task as participants in my first experiment, and, like them, were not actually intoxicated. The only obvious difference is that of expectancy: the placebo participants in my second experiment thought they had consumed alcohol and were being affected by it. However, it is not clear why this would interfere with the modulation of evaluative control by emotion. One possible explanation is that placebo participants may have attributed some of their interoceptive changes to the effects of alcohol, discouraging them from reacting to these bodily signals. Given that the magnitude of the ERN can be altered purely by the misattribution of arousal (Inzlicht and Al-Khindi, 2012), this interpretation cannot be ruled out.

However, the participants in my first experiment also likely differed from my placebo participants in a number of other ways. The participants in my first experiment were mostly first year psychology students, taking part in order to obtain course credit. The subjects in my second experiment, on the other hand, were drawn from the wider community, and were given movie vouchers for participation. Given the possible differences in motivation between these samples, and the established importance of motivational factors in error processing (e.g. Pailing \& Segalowitz, 2004), I do not believe that it is reasonable to directly compare results from these two groups. Ultimately, the lack of a no-alcohol/no-placebo control condition in my second experiment limits the inferences that can made in regard to my contrasting correlational data.

\section{Implications for Understanding the Effects of Alcohol}

Bartholow and colleagues (2012) propose that alcohol impairs post-error adaptation, and by extension, evaluative control more generally, because it reduces the negative affect generated by errors. While my results are somewhat ambiguous, and allow for multiple 
interpretations, I would nevertheless argue that the absence of any reduction in SCR or HR deceleration following errors by alcohol in my second experiment, ultimately provides strong evidence against their model. However, my results do not necessarily support Ridderinkhof and colleagues' (2002) claim that alcohol makes errors more difficult to detect either. While this model could potentially explain my lack of alcohol effects on post-error adaptation, it remains at odds with the significantly reduced FRN and post-error theta power I observed in my alcohol group participants. The mechanism by which alcohol impairs evaluative cognitive control therefore remains a mystery.

One potential alternative to existing hypotheses is that, as a $\mathrm{GABA}_{\mathrm{A}}$ agonist, alcohol may directly interfere with ACC function. Speculations as to the neurochemical basis of alcohol's effects on the ACC have typically focused on its dopaminergic properties, and the potential for this to alter basal ganglia inputs to the ACC, particularly those signalling reward prediction errors (Bartholow et al., 2012; Holroyd \& Coles, 2003). However, the ACC also contains a dense population of $\mathrm{GABA}_{\mathrm{A}}$ receptors (Northoff et al., 2007), and the direct administration of muscimol, a selective $\mathrm{GABA}_{\mathrm{A}}$ agonist, into the $\mathrm{ACC}$ has been found to disrupt both post-error slowing and the inhibition of overlearned responses in rats (Narayanan, Horst \& Laubach, 2006; Narayanan \& Laubach, 2008). Furthermore, there is evidence that Lorazepam, a benzodiazepine and selective agonist of the $\mathrm{GABA}_{\mathrm{A}}$ receptor, reduces both the amplitude of the ERN and PERI, in manner similar to alcohol (De Bruijn et al., 2004). Therefore, GABA agonism by alcohol might act to directly disrupt the normal operation of the ACC, with a dysregulation of cognitive control adjustments being just one consequence of this.

However, given the complete absence of any reduction of post-error adaption by alcohol observed in my study, we may question whether alcohol specifically impairs evaluative cognitive control at all. While it is certainly possible to interpret my findings within the 
framework of impaired evaluative control, in many ways they could be argued to be more consistent with an entirely different view on alcohol-induced cognitive deficits, namely the attention-allocation model. The attention-allocation model proposes that alcohol intoxication reduces peoples' attentional capacity, rendering them less able process the full range of stimuli they are presented with (Steele \& Josephs 1988). This causes less salient features of the environment to be ignored, potentially reducing negative affect if distraction is available to deflect attention from aversive stimuli. This narrowing of attention can also compromise inhibitory control of behaviour, if cues to withhold a response are less salient then those prompting the response.

My results appear to support the attention-allocation model in two ways. Firstly, they provide relatively strong evidence that alcohol does not alter the immediate emotional consequences of committing an error, at least when feedback is provided to make that error readily apparent. This is consistent with previous findings that alcohol does not reduce negative emotion, provided that attention cannot be easily diverted from the triggering stimulus (Curtin at al., 1998; Josephs \& Steele, 1990; Sayette et al., 2001). Secondly, I would argue that the overall increase in occipital alpha wave activity observed in my alcohol group participants is also in agreement with the attention-allocation model. Whereas a disruption of evaluative control might be reflected in a failure to produce phasic declines in alpha wave activity, a sustained increase in alpha power is more suggestive of a generally narrowed capacity for attention. Although alcohol has previously been observed to increase tonic alpha power (Lukas, Mendelson, Benedikt \& Jones, 1986; Boissoneault, Frazier, Lewis \& Nixon, 2016) to the best of my knowledge, this has never been linked to the attention-allocation model. However, given that occipital alpha waves are thought to act as a mechanism by which irrelevant stimuli may be restricted from attention (Foxe et al., 1998; Fu et al., 2001), this broad increase in alpha power following alcohol consumption, is seemingly exactly what 
the attention-allocation model would predict. It is important to note however, that the attention-allocation model is not necessarily at odds with an impairment of evaluative control by alcohol. A decrease in attentional bandwidth could potentially interact with a reduced capacity to regulate the use of cognitive control, to produce the pattern of behaviour we commonly associate with alcohol intoxication.

\section{Directions for Future Research}

The aim of this thesis was to elucidate the mechanisms underlying the impairment of post-error adaptation by alcohol, with the hope of gaining a better understanding of how alcohol might interfere with self-control outside of the lab. However, while my findings offer several new perspectives on the matter, a satisfying answer to these questions nevertheless remains elusive. There are several specific obstacles to this goal which future research might aim to overcome. Firstly, the question of whether alcohol impairs the ability to detect errors, and the extent to which this may mediate deficits in post-error adaptation, has not yet been definitively resolved. One way to address this question might be to repeat Ridderinkhof and colleagues' (2002) original experiment, while adding performance feedback on $50 \%$ of trials. If the attenuation of the ERN and PERI by alcohol, previously observed in the absence of feedback, were to disappear when errors are made obvious through feedback, this would then suggest that a reduced ability to detect errors may in fact underpin alcohol's impairment of post-error adaptation.

Secondly, although my findings offer compelling evidence that alcohol does not reduce the physiological correlates of negative affect observed following errors, it is not entirely clear whether this extends to other aspects of emotion, such as subjective experience. The evaluative priming paradigm developed by Aarts and colleagues (2012) may prove a useful tool for investigating this question. Aarts and colleagues found that when subjects failed to withhold a response in a go/no-go task, they were subsequently faster at categorizing 
negatively-valenced words than positively-valenced words. A future study might examine whether this priming effect is altered by alcohol. If we assume that intoxication does not directly reduce any form of negative affect elicited by errors, then we should expect the evaluative priming of negative categorizations to be unaffected by alcohol. However, if alcohol does in fact reduce the degree to which errors are judged as being negative, without changing initial physiological responses, we should then expect the evaluative priming effect to be weakened or entirely absent in intoxicated participants.

Thirdly, while there is substantial evidence suggesting that alcohol specifically impairs evaluative cognitive control, my findings were also arguably consistent with the attentionallocation model, in which alcohol dysregulates behaviour primarily through a narrowing of attention. Lavie's irrelevant distractor paradigm (Lavie, Hirst, De Fockert \& Viding, 2004) might offer a means to test whether alcohol principally impairs cognitive control or attentional processes. In a typical irrelevant distractor task, subjects must identify a target letters presented within an array containing multiple letters. On a certain number of trials, entirely irrelevant distractor images are presented along with the letter array. These irrelevant images nevertheless compete for subjects' attentional resources, leading to distraction in the form of increased response times. However, if the attentional load required by the letter identification task is increased, for example by increasing the variety of letters in the array, then the distraction produced by the irrelevant images is reduced. We may therefore expect that a reduction in overall attentional capacity by alcohol would have a similar effect to increasing the attentional load demanded by the task; i.e. reducing the impact of irrelevant distractors. Manipulations which prompt increased cognitive control have also been found to decrease distraction within the irrelevant distractor paradigm (e.g. Hu, Padmala, \& Pessoa, 2013; Kranz, 2015), implying that if alcohol impairs control facilities it should therefore increase distraction. Thus the effect of alcohol on performance in an irrelevant distractor task 
should, in principle, differ based on whether it interferes with attentional capacity or cognitive control, thereby providing a means to evaluate the competing claims made by these models.

\section{Conclusion}

Experience in everyday life teaches us that alcohol can have a profound impact on peoples' behaviour. However, despite the long-standing use and abuse of alcohol in our society, the reasons for this behavioural dysregulation remain a mystery. The disruption of ACC responses to errors and post-error adaption by alcohol, suggests that an impairment of evaluative cognitive control may explain many of the real-world consequences of alcohol intoxication. The aim of this thesis was to test the hypothesis that the impairment of posterror adaptation by alcohol is driven by a reduction in the negative affect normally elicited by errors. To this end I carried out two experiments. My first experiment examined physiological and behavioural changes following errors in a flanker task in sober participants, in order to assess the validity of my experimental procedure. My second experiment then applied this procedure to compare error responses in subjects receiving either an alcoholic or placebo beverage.

Data from my second experiment indicated that alcohol did not reduce SCR or HR deceleration following errors, suggesting that alcohol does not weaken the emotional impact of errors. The mechanism by which alcohol interferes with the post-error upregulation of cognitive control, and its significance for understanding the behavioural dysregulation caused by alcohol outside of the lab, therefore remains unclear. Alcohol attenuated two exploratory measures of ACC output following errors, the FRN and midfrontal theta, suggesting that the disruption of ACC function while intoxicated does not depend on emotional changes or difficulties in detecting aversive events. Furthermore, a sustained increase in occipital alpha power by alcohol raises the possibility that a narrowing of attentional capacity may contribute 
to its effects on behaviour and cognition. Further research will be needed to determine why alcohol disrupts the ACC's response to errors, and to what extent the effects of alcohol are mediated by attention. 


\section{References}

Aarts, K., De Houwer, J., \& Pourtois, G. (2012). Evidence for the automatic evaluation of self-generated actions. Cognition, 124(2), 117-127.

Alexander, W. H., \& Brown, J. W. (2010). Computational models of performance monitoring and cognitive control. Topics in Cognitive Science, 2(4), 658-677.

Allain, S., Hasbroucq, T., Burle, B., Grapperon, J., \& Vidal, F. (2004). Response monitoring without sensory feedback. Clinical Neurophysiology, 115(9), 2014-2020.

Anderson, B. M., Stevens, M. C., Meda, S. A., Jordan, K., Calhoun, V. D., \& Pearlson, G. D. (2011). Functional imaging of cognitive control during acute alcohol intoxication. Alcoholism: Clinical and Experimental Research, 35(1), 156-165.

Bailey, K., Bartholow, B. D., Saults, J. S., \& Lust, S. A. (2014). Give me just a little more time: Effects of alcohol on the failure and recovery of cognitive control. Journal of Abnormal Psychology, 123(1), 152.

Bartholow, B. D., Henry, E. A., Lust, S. A., Saults, J. S., \& Wood, P. K. (2012). Alcohol effects on performance monitoring and adjustment: affect modulation and impairment of evaluative cognitive control. Journal of Abnormal Psychology, 121(1), 173.

Bartholow, B. D., Pearson, M., Sher, K. J., Wieman, L. C., Fabiani, M., \& Gratton, G. (2003). Effects of alcohol consumption and alcohol susceptibility on cognition: a psychophysiological examination. Biological Psychology, 64(1-2), 167-190.

Başar, E. (2012). A review of alpha activity in integrative brain function: fundamental physiology, sensory coding, cognition and pathology. International Journal of Psychophysiology, 86(1), 1-24.

Bekker, E. M., Kenemans, J. L., \& Verbaten, M. N. (2005). Source analysis of the N2 in a cued Go/NoGo task. Cognitive Brain Research, 22(2), 221-231. 
Boksem, M. A., Tops, M., Wester, A. E., Meijman, T. F., \& Lorist, M. M. (2006). Error related ERP components and individual differences in punishment and reward sensitivity. Brain research, 1101(1), 92-101.

Bombeke, K., Schouppe, N., Duthoo, W., \& Notebaert, W. (2013). The effect of alcohol and placebo on post-error adjustments. Frontiers in Human Neuroscience, 7, 3.

Boissoneault, J., Sklar, A., Prather, R., \& Nixon, S. J. (2014). Acute effects of moderate alcohol on psychomotor, set shifting, and working memory function in older and younger social drinkers. Journal of studies on alcohol and drugs, 75(5), 870-879.

Boissoneault, J., Frazier, I., Lewis, B., \& Nixon, S. J. (2016). Effects of age and acute moderate alcohol administration on electrophysiological correlates of working memory maintenance. Alcoholism: Clinical and Experimental Research, 40(9), 18741883.

Botvinick, M. M., Braver, T. S., Barch, D. M., Carter, C. S., \& Cohen, J. D. (2001). Conflict monitoring and cognitive control. Psychological Review, 108(3), 624.

Boucsein, W., Fowles, D. C., Grimnes, S., Ben-Shakhar, G., Roth, W. T., Dawson, M. E., \& Filion, D. L. (2012). Publication recommendations for electrodermal measures. Psychophysiology, 49, 1017-1034.

Bradley, M. M. (2009). Natural selective attention: Orienting and emotion. Psychophysiology, 46(1), 1-11.

Bradley, M. M., Codispoti, M., Cuthbert, B. N., \& Lang, P. J. (2001). Emotion and motivation I: defensive and appetitive reactions in picture processing. Emotion, 1(3), 276.

Braver, T. S. (2012). The variable nature of cognitive control: a dual mechanisms framework. Trends in cognitive sciences, 16(2), 106-113. 
Carp, J., \& Compton, R. J. (2009). Alpha power is influenced by performance errors. Psychophysiology, 46(2), 336-343.

Carter, C. S., Braver, T. S., Barch, D. M., Botvinick, M. M., Noll, D., \& Cohen, J. D. (1998). Anterior cingulate cortex, error detection, and the online monitoring of performance. Science, 280(5364), 747-749.

Casbon, T. S., Curtin, J. J., Lang, A. R., \& Patrick, C. J. (2003). Deleterious effects of alcohol intoxication: diminished cognitive control and its Behavioural consequences. Journal of Abnormal Psychology, 112(3), 476.

Castellar, E., Kühn, S., Fias, W., \& Notebaert, W. (2010). Outcome expectancy and not accuracy determines posterror slowing: ERP support. Cognitive, Affective, \& Behavioral Neuroscience, 10(2), 270-278.

Cavanagh, J. F., \& Allen, J. J. (2008). Multiple aspects of the stress response under social evaluative threat: An electrophysiological investigation. Psychoneuroendocrinology, 33(1), 41-53.

Cavanagh, J. F., \& Frank, M. J. (2014). Frontal theta as a mechanism for cognitive control. Trends in cognitive sciences, 18(8), 414-421.

Cavanagh, J. F., \& Shackman, A. J. (2015). Frontal midline theta reflects anxiety and cognitive control: meta-analytic evidence. Journal of Physiology-Paris, 109(1-3), 3 15.

Cavanagh, J. F., Zambrano-Vazquez, L., \& Allen, J. J. (2012). Theta lingua franca: a common mid-frontal substrate for action monitoring processes. Psychophysiology, 49(2), 220-238.

Codispoti, M., Bradley, M. M., \& Lang, P. J. (2001). Affective reactions to briefly presented pictures. Psychophysiology, 38(3), 474-478. 
Compton, R. J., Bissey, B., \& Worby-Selim, S. (2014). Task motivation influences alpha suppression following errors. Psychophysiology, 51(7), 585-595.

Compton, R. J., Heaton, E. C., \& Gaines, A. (2018). Is attention enhanced following performance errors? Testing the adaptive control hypothesis. Psychophysiology, 55(4), e13022.

Crawford, J. R., \& Henry, J. D. (2004). The Positive and Negative Affect Schedule (PANAS): Construct validity, measurement properties and normative data in a large non-clinical sample. British Journal of Clinical Psychology, 43(3), 245-265.

Curtin, J. J., \& Fairchild, B. A. (2003). Alcohol and cognitive control: Implications for regulation of Behaviour during response conflict. Journal of Abnormal Psychology, 112(3), 424.

Curtin, J. J., Lang, A. R., Patrick, C. J., \& Stritzke, W. G. (1998). Alcohol and fear potentiated startle: The role of competing cognitive demands in the stress-reducing effects of intoxication. Journal of Abnormal Psychology, 107(4), 547.

Danielmeier, C., \& Ullsperger, M. (2011). Post-error adjustments. Frontiers in Psychology, 2, 233.

Debener, S., Ullsperger, M., Siegel, M., Fiehler, K., Von Cramon, D. Y., \& Engel, A. K. (2005). Trial-by-trial coupling of concurrent electroencephalogram and functional magnetic resonance imaging identifies the dynamics of performance monitoring. Journal of Neuroscience, 25(50), 11730-11737.

De Bruijn, E. R., Hulstijn, W., Verkes, R. J., Ruigt, G. S., \& Sabbe, B. G. (2004). Drug induced stimulation and suppression of action monitoring in healthy volunteers. Psychopharmacology, 177(1-2), 151-160.

Donkers, F. C., \& Van Boxtel, G. J. (2004). The N2 in go/no-go tasks reflects conflict monitoring not response inhibition. Brain and cognition, 56(2), 165-176. 
Donohue, K. F., Curtin, J. J., Patrick, C. J., \& Lang, A. R. (2007). Intoxication level and emotional response. Emotion, 7(1), 103.

Easdon, C., Izenberg, A., Armilio, M. L., Yu, H., \& Alain, C. (2005). Alcohol consumption impairs stimulus-and error-related processing during a Go/No-Go Task. Cognitive Brain Research, 25(3), 873-883.

Elkins-Brown, N., Saunders, B., He, F., \& Inzlicht, M. (2017). Stability and reliability of error-related electromyography over the corrugator with increasing trials. Psychophysiology, 54(10), 1559-1573.

Eriksen, B. A., \& Eriksen, C. W. (1974). Effects of noise letters upon the identification of a target letter in a nonsearch task. Perception \& Psychophysics, 16(1), 143-149.

Fiehler, K., Ullsperger, M., Grigutsch, M., \& von Cramon, D. Y. (2004). Cardiac responses to error processing and response conflict. Errors, conflicts, and the brain. Current opinions on performance monitoring. MPI for Human Cognitive and Brain Sciences, Leipzig, 135-140.

Fillmore, M. T., Vogel-Sprott, M., \& Gavrilescu, D. (1999). Alcohol effects on intentional 86ehaviour: Dissociating controlled and automatic influences. Experimental and Clinical Psychopharmacology, 7(4), 372.

Foxe, J. J., Simpson, G. V., \& Ahlfors, S. P. (1998). Parieto-occipital 10Hz activity reflects anticipatory state of visual attention mechanisms. Neuroreport, 9(17), 3929-3933.

Fröber, K., Stürmer, B., Frömer, R., \& Dreisbach, G. (2017). The role of affective evaluation in conflict adaptation: an LRP study. Brain and Cognition, 116, 9-16.

Fu, K. M. G., Foxe, J. J., Murray, M. M., Higgins, B. A., Javitt, D. C., \& Schroeder, C. E. (2001). Attention-dependent suppression of distracter visual input can be cross modally cued as indexed by anticipatory parieto-occipital alpha-band oscillations. Cognitive Brain Research, 12(1), 145-152. 
Gehring, W. J., Goss, B., Coles, M. G., Meyer, D. E., \& Donchin, E. (1993). A neural system for error detection and compensation. Psychological Science, 4(6), 385-390.

Gendron, M., \& Barrett, L. F. (2009). Reconstructing the past: A century of ideas about emotion in psychology. Emotion Review 1 (4): 316-39.

Gratton, G., Coles, M. G. H., \& Donchin, E. (1983). A new method for off-line removal of ocular 87ehaviou. Electroencephalography and Clinical Neurophysiology, 55(4), 468 484.

Gustafson, R., \& Kallmen, H. (1990a). Effects of alcohol on prolonged cognitive performance measured with Stroop's Color Word Test. Psychological Reports, 67(2), 643-650.

Gustafson, R., \& Källmén, H. (1990b). Alcohol and the compensation hypothesis: a test with cognitive and psychomotor tasks. Perceptual and Motor Skills, 71(3), 1367-1374.

Herrmann, M. J., Römmler, J., Ehlis, A. C., Heidrich, A., \& Fallgatter, A. J. (2004). Source localization (LORETA) of the error-related-negativity (ERN/Ne) and positivity (Pe). Cognitive Brain Research, 20(2), 294-299.

Hajcak, G., \& Foti, D. (2008). Errors are aversive: Defensive motivation and the error-related negativity. Psychological Science, 19(2), 103-108.

Hajcak, G., McDonald, N., \& Simons, R. F. (2003a). Anxiety and error-related brain activity. Biological Psychology, 64(1-2), 77-90.

Hajcak, G., McDonald, N., \& Simons, R. F. (2003b). To err is autonomic: Error-related brain potentials, ANS activity, and post-error compensatory 87ehaviour. Psychophysiology, 40(6), 895-903.

Hajcak, G., McDonald, N., \& Simons, R. F. (2004). Error-related psychophysiology and negative affect. Brain and Cognition, 56(2), 189-197. 
Hess, U., Philippot, P., \& Blairy, S. (1998). Facial reactions to emotional facial expressions: affect or cognition?. Cognition \& Emotion, 12(4), 509-531.

Holroyd, C. B., \& Yeung, N. (2003). Alcohol and error processing. Trends in Neurosciences, 26(8), 402-404.

Holroyd, C. B., \& Yeung, N. (2012). Motivation of extended behaviors by anterior cingulate cortex. Trends in cognitive sciences, 16(2), 122-128.

Hu, K., Padmala, S., \& Pessoa, L. (2013). Interactions between reward and threat during visual processing. Neuropsychologia, 51(9), 1763-1772.

Inzlicht, M., \& Al-Khindi, T. (2012). ERN and the placebo: A misattribution approach to studying the arousal properties of the error-related negativity. Journal of Experimental Psychology: General, 141(4), 799.

Inzlicht, M., Bartholow, B. D., \& Hirsh, J. B. (2015). Emotional foundations of cognitive control. Trends in Cognitive Sciences, 19(3), 126-132.

Josephs, R. A., \& Steele, C. M. (1990). The two faces of alcohol myopia: Attentional mediation of psychological stress. Journal of Abnormal Psychology, 99(2), 115.

Jonkman, L. M., Sniedt, F. L. F., \& Kemner, C. (2007). Source localization of the Nogo-N2: a developmental study. Clinical Neurophysiology, 118(5), 1069-1077.

Jung, T. P., Humphries, C., Lee, T. W., Makeig, S., McKeown, M. J., Iragui, V., \& Sejnowski, T. J. (1998). Extended ICA removes artifacts from electroencephalographic recordings. Advances in Neural Information Processing Systems (pp. 894-900).

Ker, K., \& Ivers, R. (2006). Alcohol related harm. Injury prevention, 12(4), 273-274.

Kerns, J. G., Cohen, J. D., MacDonald, A. W., Cho, R. Y., Stenger, V. A., \& Carter, C. S. (2004). Anterior cingulate conflict monitoring and adjustments in control. Science, 303(5660), 1023-1026. 
King, J. A., Korb, F. M., von Cramon, D. Y., \& Ullsperger, M. (2010). Post-error behavioural adjustments are facilitated by activation and suppression of task-relevant and task irrelevant information processing. Journal of Neuroscience, 30(38), 12759-12769.

Klimesch, W., Doppelmayr, M., Russegger, H., Pachinger, T., \& Schwaiger, J. (1998). Induced alpha band power changes in the human EEG and attention. Neuroscience Letters, 244(2), 73-76.

Kouneiher, F., Charron, S., \& Koechlin, E. (2009). Motivation and cognitive control in the human prefrontal cortex. Nature Neuroscience, 12(7), 939.

Kranz, L. S. (2015) Proactive Control of Emotional Distraction: An ERP Investigation. Victoria University of Wellington, Wellington, New Zealand.

Kypri, K., Paschall, M. J., Langley, J., Baxter, J., Cashell-Smith, M., \& Bourdeau, B. (2009). Drinking and alcohol-related harm among New Zealand university students: Findings from a national web-based survey. Alcoholism: Clinical and Experimental Research, 33(2), 307-314.

Laming, D. (1979). Choice reaction performance following an error. Acta Psychologica, 43(3), 199-224.

Lavie, N., Hirst, A., De Fockert, J. W., \& Viding, E. (2004). Load theory of selective attention and cognitive control. Journal of Experimental Psychology: General, 133(3), 339.

Levenson, R. W., Sher, K. J., Grossman, L. M., Newman, J., \& Newlin, D. B. (1980). Alcohol and stress response dampening: pharmacological effects, expectancy, and tension reduction. Journal of abnormal psychology, 89(4), 528.

Lobo, I. A., \& Harris, R. A. (2008). GABAA receptors and alcohol. Pharmacology Biochemistry and Behavior, 90(1), 90-94. 
Luck, S. J., Woodman, G. F., \& Vogel, E. K. (2000). Event-related potential studies of attention. Trends in cognitive sciences, 4(11), 432-440.

Lukas, S. E., Mendelson, J. H., Benedikt, R. A., \& Jones, B. (1986). EEG alpha activity increases during transient episodes of ethanol-induced euphoria. Pharmacology Biochemistry and Behavior, 25(4), 889-895.

Lykken, D. T., \& Venables, P. H. (1971). Direct measurement of skin conductance: A proposal for standardization. Psychophysiology, 8(5), 656-672.

Mathewson, K. E., Harrison, T. J., \& Kizuk, S. A. (2017). High and dry? Comparing active dry EEG electrodes to active and passive wet electrodes. Psychophysiology, 54(1), 74 82.

Marinkovic, K., Rickenbacher, E., Azma, S., \& Artsy, E. (2012). Acute alcohol intoxication impairs top-down regulation of Stroop incongruity as revealed by blood oxygen level-dependent functional magnetic resonance imaging. Human Brain Mapping, 33(2), 319-333.

Marinkovic, K., Rickenbacher, E., Azma, S., Artsy, E., \& Lee, A. K. (2013). Effects of acute alcohol intoxication on saccadic conflict and error processing. Psychopharmacology, 230(3), 487-497.

Mauss, I. B., Levenson, R. W., McCarter, L., Wilhelm, F. H., \& Gross, J. J. (2005). The tie that binds? Coherence among emotion experience, behavior, and physiology. Emotion, 5(2), 175.

Miller, E. K., \& Cohen, J. D. (2001). An integrative theory of prefrontal cortex function. Annual review of neuroscience, 24(1), 167-202.

Miltner, W. H., Braun, C. H., \& Coles, M. G. (1997). Event-related brain potentials following incorrect feedback in a time-estimation task: evidence for a "generic" neural system for error detection. Journal of cognitive neuroscience, 9(6), 788-798. 
Miltner, W. H., Lemke, U., Weiss, T., Holroyd, C., Scheffers, M. K., \& Coles, M. G. (2003). Implementation of error-processing in the human anterior cingulate cortex: a source analysis of the magnetic equivalent of the error-related negativity. Biological psychology, 64(1-2), 157-166.

Miyake, A., Friedman, N. P., Emerson, M. J., Witzki, A. H., Howerter, A., \& Wager, T. D. (2000). The unity and diversity of executive functions and their contributions to complex "frontal lobe" tasks: A latent variable analysis. Cognitive Psychology, 41(1), 49-100.

Moberg, C. A., \& Curtin, J. J. (2009). Alcohol selectively reduces anxiety but not fear: startle response during unpredictable versus predictable threat. Journal of abnormal psychology, 118(2), 335 .

Morey, R. D. (2008). Confidence intervals from normalized data: A correction to Cousineau (2005). Reason, 4(2), 61-64.

Narayanan, N. S., Horst, N. K., \& Laubach, M. (2006). Reversible inactivations of rat medial prefrontal cortex impair the ability to wait for a stimulus. Neuroscience, 139(3), 865 876.

Narayanan, N. S., \& Laubach, M. (2008). Neuronal correlates of post-error slowing in the rat dorsomedial prefrontal cortex. Journal of neurophysiology, 100(1), 520-525.

Nee, D. E., Wager, T. D., \& Jonides, J. (2007). Interference resolution: insights from a meta analysis of neuroimaging tasks. Cognitive, Affective, \& Behavioral Neuroscience, 7(1), 1-17.

Newman, L. A., Creer, D. J., \& McGaughy, J. A. (2015). Cognitive control and the anterior cingulate cortex: how conflicting stimuli affect attentional control in the rat. Journal of Physiology-Paris, 109(1-3), 95-103. 
Niedermeyer, E., \& da Silva, F. L. (Eds.). (2005). Electroencephalography: basic principles, clinical applications, and related fields. Lippincott Williams \& Wilkins.

Nieuwenhuis, S., Yeung, N., Van Den Wildenberg, W., \& Ridderinkhof, K. R. (2003). Electrophysiological correlates of anterior cingulate function in a go/no-go task: effects of response conflict and trial type frequency. Cognitive, affective, \& 92ehavioural neuroscience, 3(1), 17-26.

Nikolaou, K., Field, M., Critchley, H., \& Duka, T. (2013). Acute alcohol effects on attentional bias are mediated by subcortical areas associated with arousal and salience attribution. Neuropsychopharmacology, 38(7), 1365.

Nikolaou, K., Critchley, H., \& Duka, T. (2013). Alcohol affects neuronal substrates of response inhibition but not of perceptual processing of stimuli signalling a stop response. PloS One, 8(9), e76649.

Northoff, G., Walter, M., Schulte, R. F., Beck, J., Dydak, U., Henning, A., ... \& Boesiger, P. (2007). GABA concentrations in the human anterior cingulate cortex predict negative BOLD responses in fMRI. Nature neuroscience, 10(12), 1515.

Notebaert, W., Houtman, F., Van Opstal, F., Gevers, W., Fias, W., \& Verguts, T. (2009). Post-error slowing: an orienting account. Cognition, 111(2), 275-279.

O’Connell, R. G., Bellgrove, M. A., Dockree, P. M., \& Robertson, I. H. (2004). Reduced electrodermal response to errors predicts poor sustained attention performance in attention deficit hyperactivity disorder. Neuroreport, 15(16), 2535-2538.

Olvet, D. M., \& Hajcak, G. (2008). The error-related negativity (ERN) and psychopathology: toward an endophenotype. Clinical Psychology Review, 28(8), 1343-1354.

Olvet, D. M., \& Hajcak, G. (2009). The effect of trial-to-trial feedback on the error-related negativity and its relationship with anxiety. Cognitive, Affective, \& Behavioral Neuroscience, 9(4), 427-433. 
Pailing, P. E., \& Segalowitz, S. J. (2004). The error-related negativity as a state and trait measure: Motivation, personality, and ERPs in response to errors. Psychophysiology, 41(1), 84-95.

Posey, D., \& Mozayani, A. (2007). The estimation of blood alcohol concentration. Forensic Science, Medicine, and Pathology, 3(1), 33-39.

Ridderinkhof, R. K. (2002). Micro-and macro-adjustments of task set: activation and suppression in conflict tasks. Psychological research, 66(4), 312-323.

Ridderinkhof, K. R., de Vlugt, Y., Bramlage, A., Spaan, M., Elton, M., Snel, J., \& Band, G. P. (2002). Alcohol consumption impairs detection of performance errors in mediofrontal cortex. Science, 298(5601), 2209-2211.

Rose, A. K., \& Duka, T. (2008). Effects of alcohol on inhibitory processes. Behavioural Pharmacology, 19(4), 284-291.

Saunders, B., Lin, H., Milyavskaya, M., \& Inzlicht, M. (2017). The emotive nature of conflict monitoring in the medial prefrontal cortex. International Journal of Psychophysiology, 119, 31-40.

Sayette, M. A. (1993). An appraisal-disruption model of alcohol's effects on stress responses in social drinkers. Psychological Bulletin, 114(3), 459.

Sayette, M. A., Martin, C. S., Perrott, M. A., Wertz, J. M., \& Hufford, M. R. (2001). A test of the appraisal-disruption model of alcohol and stress. Journal of Studies on Alcohol, 62(2), 247-256.

Schachter, S., \& Singer, J. (1962). Cognitive, social, and physiological determinants of emotional state. Psychological Review, 69(5), 379.

Scheffers, M. K., Coles, M. G., Bernstein, P., Gehring, W. J., \& Donchin, E. (1996). Event related brain potentials and error-related processing: An analysis of incorrect responses to go and no-go stimuli. Psychophysiology, 33(1), 42-53. 
Schweizer, T. A., Vogel-Sprott, M., Danckert, J., Roy, E. A., Skakum, A., \& Broderick, C. E. (2006). Neuropsychological profile of acute alcohol intoxication during ascending and descending blood alcohol concentrations. Neuropsychopharmacology, 31(6), 1301.

Shackman, A. J., Salomons, T. V., Slagter, H. A., Fox, A. S., Winter, J. J., \& Davidson, R. J. (2011). The integration of negative affect, pain and cognitive control in the cingulate cortex. Nature Reviews Neuroscience, 12(3), 154.

Shenhav, A., Cohen, J. D., \& Botvinick, M. M. (2016). Dorsal anterior cingulate cortex and the value of control. Nature Neuroscience, 19(10), 1286.

Sher, K. J., \& Walitzer, K. S. (1986). Individual differences in the stress-response-dampening effect of alcohol: A dose-response study. Journal of Abnormal Psychology, 95(2), 159.

Sheth, S. A., Mian, M. K., Patel, S. R., Asaad, W. F., Williams, Z. M., Dougherty, D. D., ... \& Eskandar, E. N. (2012). Human dorsal anterior cingulate cortex neurons mediate ongoing behavioural adaptation. Nature, 488(7410), 218.

Somsen, R. J., Van der Molen, M. W., Jennings, J. R., \& van Beek, B. (2000). Wisconsin card sorting in adolescents: analysis of performance, response times and heart rate. Acta psychologica, 104(2), 227-257.

Steele, C. M., \& Josephs, R. A. (1988). Drinking your troubles away: II. An attention allocation model of alcohol's effect on psychological stress. Journal of Abnormal Psychology, 97(2), 196.

Steenbergen, H. V., Band, G. P., \& Hommel, B. (2009). Reward counteracts conflict adaptation: Evidence for a role of affect in executive control. Psychological Science, 20(12), 1473-1477.

Steenbergen, H. V., Band, G. P., \& Hommel, B. (2010). In the mood for adaptation: How affect regulates conflict-driven control. Psychological Science, 21(11), 1629-1634. 
Tarter, R. E., Jones, B. M., Simpson, C. D., \& Vega, A. (1971). Effects of task complexity and practice on performance during acute alcohol intoxication. Perceptual and Motor Skills, 33(1), 307-318.

Testa, M., Fillmore, M. T., Norris, J., Abbey, A., Curtin, J. J., Leonard, K. E., ... \& VanZile Tamsen, C. (2006). Understanding alcohol expectancy effects: Revisiting the placebo condition. Alcoholism: Clinical and Experimental Research, 30(2), 339-348.

Tooley, M. D., Carmel, D., Chapman, A., \& Grimshaw, G. M. (2017). Dissociating the physiological components of unconscious emotional responses. Neuroscience of Consciousness, 3(1).

Van Driel, J., Ridderinkhof, K. R., \& Cohen, M. X. (2012). Not all errors are alike: theta and alpha EEG dynamics relate to differences in error-processing dynamics. Journal of Neuroscience, 32(47), 16795-16806.

Veen, V. V., \& Carter, C. S. (2002). The timing of action-monitoring processes in the anterior cingulate cortex. Journal of cognitive neuroscience, 14(4), 593-602.

Wallstrom, G. L., Kass, R. E., Miller, A., Cohn, J. F., \& Fox, N. A. (2004). Automatic correction of ocular artifacts in the EEG: a comparison of regression-based and component-based methods. International Journal of Psychophysiology, 53(2), 105119.

Watson, D., Clark, L. A., \& Tellegen, A. (1988). Development and validation of brief measures of positive and negative affect: the PANAS scales. Journal of Personality and Social Psychology, 54(6), 1063.

Wessel, J. R., Danielmeier, C., \& Ullsperger, M. (2011). Error awareness revisited: accumulation of multimodal evidence from central and autonomic nervous systems. Journal of cognitive neuroscience, 23(10), 3021-3036. 
Wiswede, D., Münte, T. F., Goschke, T., \& Rüsseler, J. (2009). Modulation of the error related negativity by induction of short-term negative affect. Neuropsychologia, 47(1), 83-90. 


\section{Appendix A: PANAS}

This scale consists of a number of words that describe different feelings and emotions. For each word that appears, please indicate to what extent you feel this way right now. Use the following scale to record your answers:

$1=$ very slightly or not at all

$2=\mathrm{a}$ little

$3=$ moderately

4 = quite a bit

$5=$ very strongly

Record your answers by typing a number and pressing enter. If you make a mistake you can use backspace to change your answer. When you are ready to begin press enter.

interested

distressed

excited

upset

strong

guilty

scared

hostile

enthusiastic

proud 
irritable

alert

ashamed

inspired

nervous

determined

attentive

jittery

active

afraid 


\section{Appendix B: Preregistration}

Preregistration can be viewed online at https://osf.io/u6m2k/.

\section{What's the main question being asked or hypothesis being tested in this study?}

There are three main hypotheses to be tested in this study:

1) Alcohol intoxication attenuates the affective response normally elicited by an error. It is therefore predicted that intoxicated subjects will show less heart rate deceleration, smaller skin conductance responses and a smaller error-related negativity (ERN) ERP component, compared to sober subjects, following errors in a flanker task.

2) Alcohol intoxication impairs the ability to effectively up-regulate the use of cognitive control following an error (directional hypothesis). Based on this hypothesis, it is predicted that intoxicated subjects will show less reduction of interference, relative to sober subjects, following errors in a flanker task.

3) This impairment of evaluative control by alcohol is, at least in part, a function of a reduced affective response to errors (directional hypothesis). Based on this hypothesis, it is predicted that the difference in post-error reduction of interference in intoxicated and sober subjects will be significantly mediated by differences in posterror heart rate deceleration, skin conductance response and ERN size.

\section{Describe the key dependent variable(s) specifying how they will be measured}

1) Flanker Interference: Subjects will complete a letter version of the Erikson flanker task. Mean time taken to respond correctly to congruent flanker arrays (all letters the same) will be subtracted from mean time taken to respond correctly to incongruent flanker arrays (flanker letter different from target), to compute flanker interference. 
For each subject, separate indices of flanker interference will be calculated for trials following a previous correct response, and trials following a previous incorrect response. A single measure of post-error reduction of interference (PERI) will also be calculated for each subject, by subtracting flanker interference on trials following incorrect responses from flanker interference on trials following correct responses.

2) Skin Conductance Response: Subjects'skin conductance levels will be recorded continuously during the flanker task using stainless steel electrodes attached to the medial phalanges of subjects' left index and ring fingers. Skin conductance responses will be quantified as the maximum increase in SCL in the .5 to $3 \mathrm{~s}$ interval following a task response, relative to the mean SCL in the .5 s prior to the response. Increases in SCL less than $.02 \mu \mathrm{S}$ will be recorded as 0 . SCR values for each trial will be log transformed, using the formula $\log \mathrm{SCR}=\log 10(\mathrm{SCR}+1)$, and then averaged (including zeros) for each participant. Separate SCR magnitudes will be calculated for correct responses and for incorrect responses.

3) Heart Rate: Subjects' heart rates will be recorded continuously during the flanker task using $\mathrm{Ag} / \mathrm{AgCl}$ electrodes placed on the right shoulder and lower left ribcage, referenced to an electrode placed on the left shoulder. Heart rate will be defined as the interval between successive R-wave spikes, converted to beats per minute. To measure change in HR following flanker task responses, the $3 \mathrm{~s}$ following each response will be divided into $.5 \mathrm{~s}$ intervals. Mean $\mathrm{HR}$ in the $.5 \mathrm{~s}$ prior to a response will then be subtracted from the mean HR within each of these intervals. Separate HR change values will be calculated for correct responses and for incorrect responses.

4) Error-Related Negativity: Electroencephalography recording will take place continuously during the flanker task using scalp electrodes arranged according to the international 10-20 system. Scalp electrodes will be referenced to the average value of 
electrodes placed on the left and right mastoid bones. The ERN will be quantified as the most negative value at the electrodes Fz, FCz and $\mathrm{Cz}$, in the $100 \mathrm{~ms}$ following a task response. Separate ERN values will be calculated for correct responses and for incorrect responses.

\section{How many and which conditions will participants be assigned to?}

Participants will be assigned to one of two beverage conditions: an alcohol condition, in which subjects consume a quantity of an alcoholic beverage calculated to produce a blood alcohol level of $.08 \mathrm{~g} / 100 \mathrm{ml}$, and a placebo condition, in which subjects consume a sham alcoholic beverage of equivalent volume.

\section{Specify exactly which analyses you will conduct to examine the main} question/hypothesis.

To test the first hypothesis three ANOVA will be carried out:

1) SCR will be analysed in a 2 (beverage group: alcohol, placebo) X 2 (response type: correct response, incorrect response) mixed factorial ANOVA. If an interaction is found between beverage group and response type, a follow-up independent samples ttest (one-way) will be carried out to compare SCR to incorrect responses only, between the alcohol and placebo groups.

2) HR change will be analysed in a 2 (beverage group: alcohol, placebo) $X 2$ (response type: correct response, incorrect response) X 6 (time) ANOVA. If an interaction is found between beverage group and response type, or if a three-way interaction is found, a series of follow-up independent samples t-tests (one-way) will be carried out, comparing HR change to incorrect responses between the alcohol and placebo groups, for each time point separately. However, if HR change following correct responses 
differs significantly between alcohol and placebo groups, as determined by a 2 (beverage group) X 6 (time) ANOVA, then the difference in HR change between correct and incorrect responses will be used as the dependent variable in follow-up tests, rather than HR change to incorrect responses.

3) The ERN will be analysed in a 2 (beverage group: alcohol, placebo) $X 2$ (response type: correct response, incorrect response) mixed factorial ANOVA. If an interaction is found between beverage group and response type, a follow-up independent samples t-test (one-way) will be carried out to ERN size for incorrect responses only, between the alcohol and placebo groups.

To test the second hypothesis, flanker interference will be analysed in a 2 (beverage group: alcohol, placebo) X 2 (trial type: previous trial correct, previous trial incorrect) mixed factorial ANOVA. If an interaction is found between beverage group and trial type, a followup independent samples t-test (one-way) will be used to compare mean PERI between alcohol and placebo groups.

If the first and second hypotheses are supported, the third hypothesis will be tested using structural equation modelling. A separate mediation model will be constructed for each physiological variable for which error responses differ significantly between alcohol and placebo participants. In each model, beverage group (dummy coded as 0 or 1) will act as the independent variable, PERI will act as the dependent variable, and the physiological variable of interest (SCR to incorrect responses, HR change to incorrect responses, ERN to incorrect responses) will act as the mediator variable. As with follow-up t-tests, difference in HR change between correct and incorrect responses will replace HR change following incorrect responses, if HR change following correct responses differs between beverage groups. The time point used for HR analysis will be the time point at which the largest bivariate 
correlation between HR change and PERI occurs, for which a significant difference between alcohol and placebo groups is also found.

For all statistical tests, p-values less than .05 will be regarded as significant. P-values between .05 and .1 will be treated as marginally significant. If a predicted interaction in an ANOVA reaches marginal significance, follow-up t-tests will still be carried out. Statistical significance for mediation analyses will determined using the bootstrap method.

How many observations will be collected or what will determine sample size? No need to justify decision, but be precise about exactly how the number will be determined. Analyses will use data collected from 80 subjects in total, 40 from each beverage group. Subjects' data will be excluded from analyses if they have an overall accuracy of $<.66$ in the flanker task or if they make fewer than 10 incorrect responses in the flanker task. If a subject is excluded, an additional subject will be recruited to replace them, so that the target sample size of 80 is reached. On the other hand, if a subjects' data for one or more physiological measures is unusable for technical reasons (e.g. equipment malfunction, excess artifacts), they will be excluded only from analyses involving that measure. 
